\title{
Estimation of particle impact based erosion using a coupled direct particle - compressible gas computational fluid dynamics model
}

\author{
L.A. Florio \\ US Army ARDEC \\ Technology Branch \\ Small Caliber Armaments Division \\ Picatinny Arsenal, NJ 07806 \\ Phone:(973)724-5993 \\ Fax: (973) 724-2419 \\ Email:laurie.a.florio.civ@mail.mil
}

\begin{abstract}
:
A comprehensive two-phase compressible gas-solid particle computational fluid dynamics-based modeling method has been developed and applied to study the erosion and fouling along flow path surfaces due to particle impact. Extending upon methods to predict particle interaction phenomena which incorporate rolling, twisting, sliding, and adhesion forces to capture conditions that frequently occur as particle laden flow passes over a flow path surface, the simulation methods have been specifically designed for high particle concentrations and large particle sizes. In the developed technique, individual particles are directly discretized in the computational mesh, and the particles move through the fluid, and interact with the flow, flow path boundaries, and other particles. With particle motion calculated from the direct two-phase flow simulations, the distribution and intensity of the mass loss or erosion conditions along a flow path surface resulting from particle impact is predicted. The developed method was applied to study the erosion of an aluminum wall due to impacting sand particles of different sizes, impact velocities, and counts. A more fluid-like particle motion and distribution upon impact were found as the particle size decreased. The method was also implemented to examine the erosion that develops for three common flow configurations which force particle-flow path surface interactions: a diverging/converging flow path, a bypass flow path, and a baffle configuration. These applications demonstrate the utility of the model to explore and better understand the relationships between the geometry, the flow, the particles, and the adhesion and erosion that develop as particle laden flow moves over the flow path surfaces.
\end{abstract}

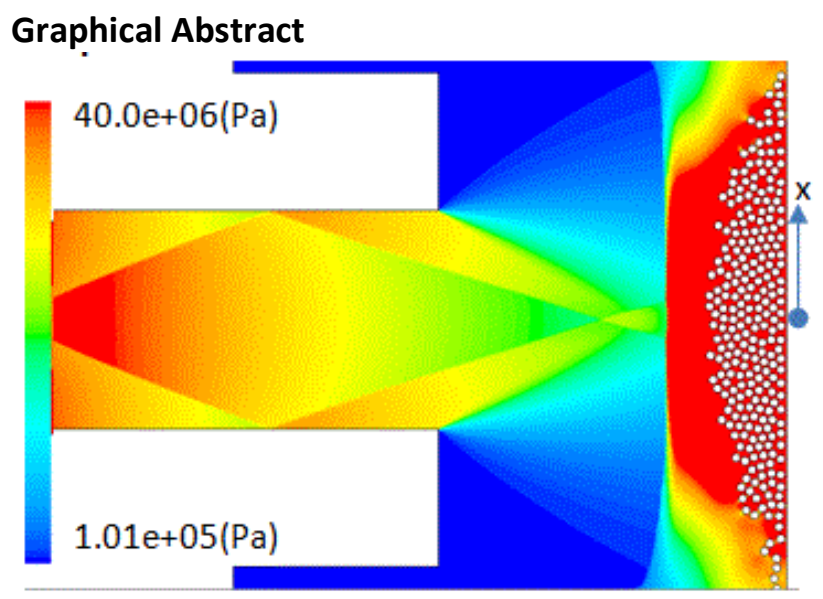

Pressure distribution as particles impact

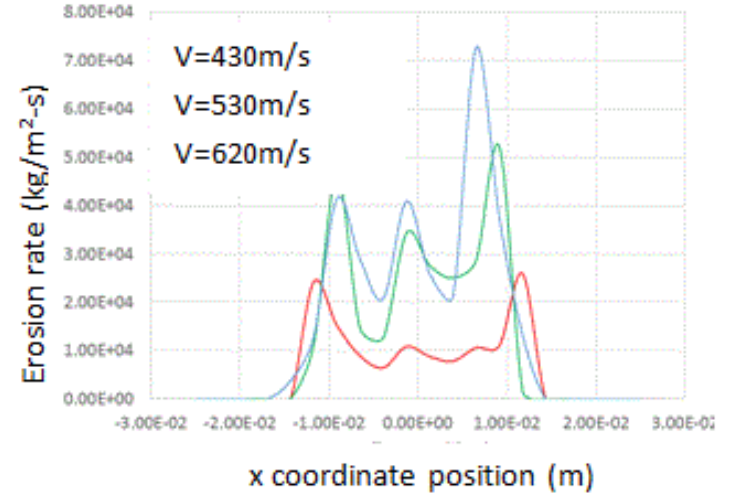

Erosion rate distribution along impact surface Flow driven particles impacting a normal plate 


\section{List of Symbols:}

\begin{tabular}{|c|c|c|}
\hline Vectors & & Description \\
\hline Eq. & & \\
\hline C & $=$ & Net tangential displacement/rotation \\
\hline d & $=$ & Vector from particle 1 to particle 2 \\
\hline$E$ & $=$ & Quaternion \\
\hline$\dot{\boldsymbol{E}}$ & $=$ & Time derivative of quaternion \\
\hline $\boldsymbol{F}$ & $=$ & Force(N) \\
\hline$F_{n}$ & $=$ & Normal force vector due to collision(N) \\
\hline $\boldsymbol{F}_{t}$ & $=$ & Tangential force vector due to collision(N) \\
\hline I & $=$ & Moment of inertia matrix \\
\hline$M$ & $=$ & Moment(N/m) \\
\hline $\mathbf{n}$ & $=$ & Unit normal vector \\
\hline $\mathbf{r}$ & $=$ & $\begin{array}{l}\text { Particle center of gravity to contact position } \\
\text { vector figure } 4(\mathrm{~m})\end{array}$ \\
\hline$T_{r o}$ & $=$ & Rolling direction \\
\hline$t_{\text {tang }}$ & $=$ & Unit tangential vector \\
\hline$t_{T w}$ & $=$ & Twisting direction \\
\hline $\boldsymbol{v}$ & $=$ & Fluid velocity (m/s) \\
\hline$V_{o}$ & $=$ & Solid body velocity $(\mathrm{m} / \mathrm{s})$ \\
\hline$V_{n}$ & $=$ & Normal component of relative velocity \\
\hline $\boldsymbol{v}_{\mathrm{R}}$ & $=$ & Relative velocity with respect to particle $1(\mathrm{~m} / \mathrm{s})$ \\
\hline$v_{R O}$ & $=$ & Relative rolling velocity (m/s) \\
\hline$V_{t}$ & $=$ & Tangential component of relative velocity \\
\hline$v_{T W}$ & $=$ & Relative twisting velocity \\
\hline$\Omega$ & $=$ & Angular velocity(rad/s) \\
\hline$\dot{\mathbf{\Omega}}$ & $=$ & Angular acceleration $\left(\mathrm{rad} / \mathrm{s}^{2}\right)$ \\
\hline$x$ & $=$ & Coordinate directions \\
\hline$x_{1}, x_{2}$ & $=$ & Center of gravity positions of particle 1 and $2(\mathrm{~m})$ \\
\hline
\end{tabular}

Scalars

$\begin{array}{ccc}B H & = & \text { Brinell Hardness } \\ C, n_{1} n_{1}, n_{2,} & = & \text { Constants } \\ n_{3} & & \text { Turbulence parameters } \\ C, G, Y, S & = & \text { Overlap area for particles } \\ c_{-} a & = & \text { Length factor } \\ c-f & = & \text { Contact area for particles } \\ c \_l & = & \text { Parameter for determining collision } \\ C Z & = & \text { Empirical constant } \\ \mathrm{E} & = & \text { Erosion rate }\left(\mathrm{kg} / \mathrm{m}^{2}-\mathrm{s}\right) \\ \dot{e} & = & \text { Erosion ratio }\end{array}$




\begin{tabular}{|c|c|c|c|}
\hline$f$ & $=$ & Maximum value of $F$ & $(40)$ \\
\hline$F$ & $=$ & Function of impact angle & (40) \\
\hline$F$ & $=$ & Force(N) & $(5,28-30,35,36)$ \\
\hline$F_{S}$ & $=$ & Sharpness factor & (36) \\
\hline$G$ & $=$ & Damper coefficient(varies) & $(19-22)$ \\
\hline$h$ & $=$ & Gas enthalpy(J/kg-K) & (3) \\
\hline$H_{v}$ & $=$ & Vickers hardness & (37) \\
\hline$k$ & $=$ & Thermal conductivity $(\mathrm{W} / \mathrm{m}-\mathrm{K})$ & (3) \\
\hline$k$ & $=$ & Spring constant(varies) & $(21-24)$ \\
\hline$M$ & $=$ & Moments(N-m) & $(31-34,37)$ \\
\hline$m_{0}$ & $=$ & Mass(kg) & (34) \\
\hline$p$ & $=$ & Pressure $\left(\mathrm{N} / \mathrm{m}^{2}\right)$ & $(2,5)$ \\
\hline$R$ & $=$ & Particle radius figure $4(\mathrm{~m})$ & $(7,8)$ \\
\hline$S$ & $=$ & Particle clearance (m) & (7) \\
\hline$T$ & $=$ & Temperature(K) & (3) \\
\hline$t$ & $=$ & Time(s) & \\
\hline$U^{*}$ & $=$ & Turbulent dimensionless velocity & (6) \\
\hline$U_{p}$ & $=$ & Mean velocity at cell next to wall $(\mathrm{m} / \mathrm{s})$ & (6) \\
\hline$y^{*}$ & $=$ & Turbulent dimensionless distance & (6) \\
\hline$Y p$ & & Normal distance to centroid of wall cell (m) & (6) \\
\hline \multicolumn{4}{|c|}{ ( } \\
\hline$\beta$ & $=$ & Fraction of normal force for cohesion & (35) \\
\hline$k, \varepsilon$ & $=$ & Turbulence parameters & (4) \\
\hline$\kappa_{P}$ & $=$ & Mean turbulence parameter at cell next to wall & (6) \\
\hline$\kappa V$ & $=$ & Von Karman constant & (6) \\
\hline$\rho$ & $=$ & Fluid density $\left(\mathrm{kg} / \mathrm{m}^{3}\right)$ & $(1,2,3,4)$ \\
\hline$\rho$ & $=$ & Particle density $\left(\mathrm{kg} / \mathrm{m}^{3}\right)$ & (41) \\
\hline$\tau$ & $=$ & Stress tensor $\left(\mathrm{N} / \mathrm{m}^{2}\right)$ & $(2,3,5,6)$ \\
\hline$\mu$ & $=$ & Frictional coefficient & $(30,32)$ \\
\hline$\mu$ & $=$ & Dynamic fluid viscosity & $(4,6)$ \\
\hline \multicolumn{4}{|c|}{ Notation } \\
\hline $\mathrm{B}$ & $=$ & Body, local coordinate system & $(37)$ \\
\hline coh & $=$ & Associated with cohesion & \\
\hline $\mathrm{n}$ & $=$ & Associated with normal & \\
\hline P1, P2 & $=$ & Associated with particle 1 and particle 2 & \\
\hline RO & $=$ & Associated with rolling & \\
\hline $\mathrm{R}^{\mathrm{xG}}$ & $=$ & Rotational matrix from local csys $x$ to global & (A.1) \\
\hline $\mathrm{T}$ & $=$ & Associated with tangential & \\
\hline $\mathrm{t}$ & $=$ & Associated with tangential & \\
\hline TW & $=$ & Associated with twisting & \\
\hline w & $=$ & Associated with wall & $(5,6)$ \\
\hline${ }^{B}$ Z & $=$ & Variable notation, in terms of coordinate system B & $(37)$ \\
\hline
\end{tabular}


$\mathrm{Z}^{\mathrm{x}} \quad=\quad$ Variable notation, associated with object $\mathrm{x} \quad$ (A.1)

$Z_{x}^{x} \quad=\quad$ Variable notation, object $\mathrm{x}$, coordinate system $\mathrm{x} \quad$ (A.2) 


\subsection{Introduction}

High speed compressible flow often impinges upon solid surfaces, and, when this flow carries particulates, the impact can lead to the erosion or wearing of the solid surface material. The particle impact induced wear phenomenon has a wide range of applications from industrial processes to turbines to aerodynamic flows, both internal and external. Since this wear can alter the flow path geometry as well as the material properties and structural integrity of the solid body flow path, the ability to simulate, analyze, and investigate the distribution of the erosion rates and the effects that the flow, particle, and surface conditions have on the erosion development is important in assuring that a system maintains proper and safe operation.

Particle-gas flow and particle impact based erosion simulations commonly employ assumptions compatible with dilute particle concentrations, which neglect particle-particle interactions and the effects of the volume of fluid occupied by the particles, and the particle-flow momentum exchange. An earlier work by McLaury [1] couples computational fluid dynamics with particle impact based erosion modeling. Particle motion is tracked by a Lagrangian method and coefficients of drag are used to estimate the fluid induced forces. The method is applied to investigate the erosion conditions in a pipe bend. Among recent investigations implementing a dilute particle concentration assumption include a study by Kim [2], involving Lagrangian particle tracking within a commercial computational fluid dynamics code to examine erosion of a coated specimen caused by $1 \mu \mathrm{m}$ to $4 \mu \mathrm{m}$ diameter particles, with comparisons made to physical test results. Graham et al.[3] studied the wear distributions along a pipe elbow and with a cylindrical protrusion in the pipe resulting from particles impacting the surfaces of the elbow both numerically, under a dilute assumption, and experimentally. Erosion rate distributions were measured using a coordinate measuring machine and compared to dilute concentration based model results, with differences attributed to neglecting the change in the flow path geometry caused by the erosion. Solnordal [4] and Wong [5] indicate that larger particles may have more influence on the erosion since smaller particles often do not attain sufficient energy to impact the flow path / eroding surfaces.

These dilute assumptions are not valid for many multiphase flow conditions as the particle concentrations and/or size may be large enough that the particle contacts are frequent and particle volumes and momentum exchange with the fluid are significant. One means that has been devised to examine such non-dilute operating conditions is the coupling of discrete element models (DEM) to simulate the particle dynamics, including particle interactions, and computational fluid dynamics (CFD) models to simulate the flow conditions and determine the fluid induced forces acting on the 
particles. Typically, in such simulations, the particles are not fully discretized in the CFD models, but a particle volume fraction or void fraction is returned to the CFD models based on the particle positioning predicted by the DEM models. Drag correlations are used to estimate the fluid induced forces, with soft collision methods commonly used to model the contact forces between objects in the system. Various interaction models have been developed for different particle materials and collision regimes such as elastic or elastic-plastic collisions. Additional phenomena have also been incorporated into these models, including the drying processes, pyrolysis, chemistry [6] and erosion. One of the early studies incorporating the particle interaction effects along with particle laden flow is the work of Tsuji et al. [7] who investigated the flow of particles in a horizontal pipe, tracking the motion of each particle and using a modified Cundall-Strack type interaction method. Chen et al. [8] investigated the erosion in a pipe elbow caused by $300 \mu \mathrm{m}$ diameter particles carried with a liquid by employing a discrete element method model. Similarly, Jafari et al. [9] conducted a parametric study on the effect of annular pipe dimensions on the erosion in the pipe due to particle collisions with particle interactions modeled.

While a greater range of phenomena can be analyzed with these CFD-DEM models when compared to the use of a dilute Lagrangian particle tracking model, such techniques still cannot capture many localized effects that develop in particle laden flow processes. These events include particle sticking, particle build-up along surfaces, particle resuspension into the flow, loss of particles/material from the surfaces, and particle-particle or particle-wall interactions, particularly at lower relative speeds and when rolling or twisting between particles or particles and flow path surfaces are involved. Such phenomena are particularly important when studying erosion and fouling events which are driven by particle-particle and particle-wall interaction effects. When such details are needed to understand and investigate a system or phenomenon, alternative simulation techniques are necessary.

This work describes a novel method for modeling particle based erosion and fouling phenomena that is able to capture the related effects in greater detail than commonly used techniques. While the focus of this work is the erosion prediction, the capabilities for including the adhesion or cohesion effects have been developed since the particle flow path surface interactions and thus the erosion are affected by the particle build-up along the surfaces. A direct particle-flow coupling method, developed by the author [10-12], with particles directly placed within the computational domain, is extended to include these new capabilities. Local flow conditions around each particle that can directly account for the effects of nearby particles, walls, or other surfaces are used to 
estimate the fluid induced forces acting on each particle. Particle-particle or particle-wall interaction modeling is handled through a soft collision model that now have been made more comprehensive to incorporate rolling, twisting, sliding, and adhesion effects that are important in erosion and fouling events. The resulting rotational and translational motion of each particle is then determined. Once the particle motion is known, the particle position and velocity can be used to estimate the erosion rate distribution over a flow path surface resulting from the impacting particles. After describing and demonstrating the modeling methods and capabilities, the techniques developed are then applied to study the effect of particle size and impact velocity on the particle based erosion distributions along a normal wall (figure 1). Then the erosion distributions are predicted for three general systems with flow path geometries that promote particle impact based erosion and that are commonly found in industrial systems (figure 2). The study demonstrates the clear advantages and improvements the developed method offers over standard Lagrangian based methods in the ability to explore and understand the interrelationships between the flow conditions, the system geometries, the flow path and particle conditions and motion and the erosion patterns that develop compared to the information gained from.

\subsection{Modeling technique}

The direct particle-flow modeling technique involves direct particle kinematics - computational fluid dynamics coupling [10-12]. The particles are completely discretized within the computational fluid dynamics domain (figure 3) with the kinematics model informing the computational fluid dynamics model of the translational and rotational position and velocity of each of the particles. Hence, the fluid structure interaction is directly simulated and allows for the unique capabilities of the developed modeling technique. In this work, improvements are made to the particle-particle-wall interaction models to include additional effects that become increasingly significant as particle-particle interactions occur at lower speeds and during particle-wall interactions. Previous development of this method by the author was focused mainly on high speed collisions between particles in high speed compressible flow [10-12]. However, in the current work, a general and adjustable method is presented that may be useful for general flow regime conditions. While the developed method can also be used to study the accretion and agglomeration of particles, the focus in this study is on the prediction of the particle impact based erosion.

The modeling technique employed requires three main components: a computational fluid dynamics based model with moving particles to directly predict the flow conditions, a particle kinematics based model to determine the translational and rotational motion of the individual 
particles as they interact and move through the fluid, and an erosion rate model to predict the erosion due to particle impact that occurs for specific material and collision conditions. Each of these three areas is described in more detail below.

\subsection{Fluid-flow modeling}

The first component of the modeling technique is the fluid flow model. The computational fluid dynamics model also serves as central driver of the technique as the two remaining components are coded into subroutines executed from the CFD model. The Navier-Stokes equations for compressible gas flow are solved using computational fluid dynamics (CFD) based methods with moving particles within the computational domain. The method described is not limited to a particular flow regime or fluid type. Multispecies gas mixtures, chemical reactions [13], multiphase flows, phase change, additional sources and turbulence can be incorporated into the simulations. For the conditions considered in this work, turbulent, single phase compressible gas flow is considered.

The conservation of mass, momentum, and energy along with an equation of state for the fluid and turbulence parameter governing equations are used to determine the fluid velocity, pressure, and temperature. The conservation equations solved as found in [14-15] are:

1. Conservation of mass, with ideal gas air in the current model, is given by:

$$
\partial \rho / \partial t+\nabla \cdot(\rho \bar{v})=0
$$

2. Conservation of momentum is given by:

$$
\rho D \bar{v} / D t=\nabla \cdot \tau_{i j}-\nabla p
$$

3. Conservation of energy is expressed as:

$$
\rho D h / D t=D p / D t+\nabla \cdot(k \nabla \mathrm{T})+\tau_{i j} \partial u_{i} / \partial x_{j}
$$

4. With the high speed flows involved, a $\kappa-\varepsilon$ realizable turbulence model is employed [15] as determined from the two equations below. The turbulence model was selected for its stability in the flow conditions of interest. However, the method is not limited to a specific turbulence model or flow regime and would be just as applicable for laminar flows, turbulent flows, or any other viscous model.

$$
\begin{gathered}
\left(\frac{\partial}{\partial t}(\rho k)+\frac{\partial}{\partial x_{i}}\left(\rho k v_{i}\right)\right)=\frac{\partial}{\partial x_{j}}\left(\left(\mu+\frac{\mu_{t}}{\sigma_{k}}\right) \frac{\partial k}{\partial x_{j}}\right)+G_{k}+G_{b}-\rho \varepsilon-Y_{M}+S_{k} \\
\left(\frac{\partial}{\partial t}(\rho \varepsilon)+\frac{\partial}{\partial x_{i}}\left(\rho \varepsilon v_{i}\right)\right)=\frac{\partial}{\partial x_{j}}\left(\left(\mu+\frac{\mu_{t}}{\sigma_{\varepsilon}}\right) \frac{\partial \varepsilon}{\partial x_{j}}\right)+\rho C_{1} S_{\varepsilon}-\rho C_{2}\left(\frac{\varepsilon^{2}}{\kappa+\sqrt{v \varepsilon}}\right)+C_{1 \varepsilon} \frac{\varepsilon}{\kappa} C_{3 \varepsilon} G_{b}+S_{\varepsilon}
\end{gathered}
$$

The governing equations can be found in White and Shih [14-15]. 
The commercial finite volume based computational fluid dynamics modeling software FLUENT $\odot$, Release 16 [16], is used to carry out the general flow solutions, to move the mesh which contains the particles, and to issue commands to execute author generated subroutines that carry out particle kinematic and particle impact based erosion modeling.

\subsection{Particle kinematics}

The translational and rotational motion of each moving particle/object in the system is calculated based on the forces/moments acting on the body. The loading acting on each particle may include the fluid induced forces, $\mathrm{F}_{\text {fluid }}$, the gravitational forces, $\mathrm{F}_{\text {grav }}$, the particle interaction forces including a normal, tangential, and cohesion or adhesion force, $\mathrm{F}_{\mathrm{n}}, \mathrm{F}_{\mathrm{t}}$, and $\mathrm{F}_{\mathrm{coh}}$, respectively, and any other forces that can be expressed in terms of standard mathematical functions. In the current work, all particles are assumed to be circular/spherical. Non-circular/non-spherical particles may be treated in a similar manner, but with changes in the contact normal, moment of inertia, center of gravity, contact location position, and other expressions. While [10-12] focused on high speed flows and collisions, this work incorporates additional contact phenomena, particularly the relative particle rolling, twisting and sliding effects and the cohesion/adhesion effects. In addition, the contact force expressions used in this work are more amenable to lower speed collisions that may specifically occur during erosion and fouling.

\subsubsection{Fluid induced forces}

The fluid induced loading is found by utilizing the local fluid flow conditions at the surface of the particle/object. The local numerical based pressure and viscous forces are taken into account as in equation 5.

$$
F_{\text {fluid }}=\oiint_{\text {Asurf }} \boldsymbol{\tau}_{\omega} \boldsymbol{d} \boldsymbol{A}_{\text {surf }}+\oiint_{\text {An }} \boldsymbol{P} \boldsymbol{d} \boldsymbol{A}_{\boldsymbol{n}}
$$

For the $\kappa-\varepsilon$ turbulence model, the shear stress at the wall, $\tau_{\mathrm{w}}$, is determined from the following:

$$
\tau_{w}=\frac{\kappa_{v} \rho C_{\mu}^{1 / 4} k_{p}^{1 / 2}}{\ln \left(E y^{*}\right)} ; \quad y^{*}=\frac{\rho C_{\mu}^{1 / 4} k_{p}^{1 / 2} y_{P}}{\mu}
$$

where P stands for the conditions at the centroid of the fluid cell adjacent to the wall. If other viscous models are used, modified wall shear stress formulations can be substituted. Then, the appropriate moments resulting from the fluid induced forces can be determined, knowing the center of gravity location within the particle. In this way, empirically based correlation equations are not required to estimate the fluid induced forces. 


\subsubsection{Particle interaction models}

2.2.2.1 Particle collision detection. Particle interaction forces including contact/collision and cohesion forces depend on the relative position or motion of the particles. Contact forces are only applied when the two objects are defined to be colliding. The determination of the contact or collision status of pairs of particles or a particle and a wall is made through the calculation of the distance between the particle pairs or particle and a wall. The proper determination of the collision status and the application of the appropriate collision force is particularly critical in the direct CFDDEM type coupling to prevent mesh overalap because the mesh topology of the CFD model cannot be altered. Some gap clearance space, S, must be maintained between any particle pair or between a particle and a wall (figure 4). A portion of the clearance space is a layer near the particle boundary that travels with the particle to assist in the resolution of the flow near the particle walls as seen in figure $3 \mathrm{~b}$. In the current formulation, all particles are assumed to be of a circular or spherical shape. When the distance, $|\bar{d}|$, figure 4 , between the centers of particle $\mathrm{P}_{1}$ of radius $\mathrm{R}_{1}$ and particle $\mathrm{P}_{2}$ of radius $R_{2}$, becomes less than the sum of the two particle radii and the required clearance space, $S$, the particles are said to be colliding. The distance and the collision status of a set of particles are tracked through a parameter, $\mathrm{CZ}$, defined in equation 7.

$$
C Z=R_{1}+R_{2}+S-|\bar{d}| ; \bar{d}=\overline{x_{2}}-\overline{x_{1}} \quad ; \bar{n}=\frac{\bar{d}}{|\bar{d}|}
$$

When CZ is greater than zero, the particle pair is colliding. While the current parameter definitions are given for circular/spherical particles shapes, the same basic principles can be applied to noncircular/spherical shapes, with more complex formulations for particle center of gravity, surface normals, and contact locations than those presented in the current work.

Improvements were made to the efficiency in the collision search procedure compared to the basic search procedures carried out in [10-12]. First, surface normals of the walls were directly calculated rather than using the decomposition of a surface into a set of circles/spheres. Second, collisions searches were restricted to particles assigned to particular neighboring "bins" rather than the entire domain, following the binning methods described by Matuttis and Chen [17]. Together these two changes resulted in about a $25 \%$ decrease in run time, though the specific calculations that brought about the speed-up cannot be determined.

2.2.2.2 Particle collision contact forces and moments. Under conditions where the particles or particles and walls are defined to be colliding, the proper forces and moments must be applied to replicate the effects of the contact between the two objects. The force formulation below is 
independent of flow regime. Adjustments in the coefficients/parameters may be required for a particular mesh size or relative particle speed to prevent the mesh overlap or for expected particle motion. In the current work, soft collision type models are implemented since this technique can readily represent simultaneous collisions between multiple objects. The soft collision model uses sets of spring-damper type force formulations to represent the various modes of particle-particle and particle-wall interactions. Normal collision forces, tangential collision forces, rolling and twisting related torques or moments, and adhesion/cohesion effects are considered in the interactions models implemented in the current work. These are based on the formulations of Matuttis and Chen [17] and Marshall and Li [18].

Before describing the force and moment models, a number of scalar and vector quantities must be defined. Let $\bar{n}$ be a unit vector pointing from the center of particle 1 of radius $\mathrm{R}_{1}$, situated at $\overline{x_{1}}$, to the center of particle 2 of radius $\mathrm{R}_{2}$, situated at $\overline{x_{2}}$, (figure 4 ) then

$$
\overline{r_{1}}=R_{1} \bar{n} \text { and } \overline{r_{2}}=-R_{2} \bar{n}
$$

If $\overline{v_{1}}$ and $\overline{v_{2}}$ are the translational velocities of particle 1 and particle 2 respectively and $\overline{\Omega_{1}}$ and $\overline{\Omega_{2}}$ are the angular velocities of particle 1 and particle 2 respectively, then the relative particle velocity or approach velocity at the point of contact, $\overline{v_{R}}$, is given by:

$$
\overline{v_{R}}=\overline{v_{1}}-\overline{v_{2}}+\left\{\left(\overline{\Omega_{1}} \times \overline{r_{1}}\right)-\left(\overline{\Omega_{2}} \times \overline{r_{2}}\right)\right\}
$$

The normal component of the relative velocity at the point of contact, $\bar{v}_{n}$, is then:

$$
\bar{v}_{n}=\left(\overline{v_{R}} \cdot \bar{n}\right) \bar{n}
$$

The tangent component of the relative velocity at the point of contact, $\bar{v}_{t}$, is then:

$$
\bar{v}_{t}=\bar{v}_{R}-\bar{v}_{n}
$$

The tangent $\bar{t}_{\text {tang }}$ direction is defined as :

$$
\bar{t}_{\text {tang }}=\bar{v}_{t} /\left|\bar{v}_{t}\right|
$$

The relative rolling velocity of the two particles, $\bar{v}_{R O}$, as in [18] is equal to:

$$
\bar{v}_{R O}=\frac{1}{2}\left(-\overline{v_{R}}+\overline{v_{1}}-\overline{v_{2}}-\frac{\left(R_{1}-R_{2}\right)}{\left(R_{1}+R_{2}\right)}\left\{\left(\bar{n} \times\left(\overline{v_{1}}-\overline{v_{2}}\right)\right) \times \bar{n}\right\}\right)
$$

The rolling direction, $\bar{t}_{r o}$ is defined as :

$$
\bar{t}_{r o}=\bar{v}_{R O} /\left|\bar{v}_{R O}\right|
$$


Finally, the relative twisting velocity between the particles at the point of contact, $\bar{v}_{T W}$, as in [18] is equal to:

$$
\bar{v}_{T W}=\left(\bar{n} \cdot\left(\overline{\Omega_{1}}-\overline{\Omega_{2}}\right)\right) \bar{n}
$$

The twisting direction, $\bar{t}_{T W}$, is defined as :

$$
\bar{t}_{T W}=\bar{v}_{T W} /\left|\bar{v}_{T W}\right|
$$

Defining a length factor for the particles in contact of $c_{-} f$ as in [17]:

$$
C_{-} f=\frac{4 R_{1} R_{2}}{\left(R_{1}+R_{2}\right)}
$$

Defining a contact overlap area for particles in contact of $c \_a$ :

$$
c_{-} a=R_{1}^{2} \operatorname{acos}\left(\frac{R_{1}-C Z}{R_{1}}\right)-\left(R_{1}-C Z\right) \sqrt{2 R_{1} C Z-C Z^{2}}
$$

Defining a contact area for the particles in contact of $c_{-} l$ :

$$
c_{-} l=2 \sqrt{2 R_{1} C Z-C Z^{2}}
$$

Defining an equivalent mass, Young's Modulus, Bulk Modulus, and Radius as in [18]:

$$
m_{\mathrm{eq}}=\frac{m_{1} m_{2}}{\left(m_{1}+m_{2}\right)} ; E_{\mathrm{eq}}=\frac{E_{1} E_{2}}{\left(E_{1}+E_{2}\right)} ; G_{\mathrm{eq}}=\frac{G_{1} G_{2}}{\left(G_{1}+G_{2}\right)} ; R_{\mathrm{eq}}=\frac{R_{1} R_{2}}{\left(R_{1}+R_{2}\right)} \quad \text { Eq.(20) }
$$

The following spring parameters, labeled K, and damping parameters, labeled G, are assigned for each of the modes of collision:

$$
\begin{gathered}
\text { Normal Mode: } K_{\mathrm{N}}=\mathrm{E}_{\mathrm{eq}} \frac{c_{-} a}{c_{-} f} \quad G_{\mathrm{N}}=\sqrt{2 \mathrm{E}_{\mathrm{eq}} \frac{c_{-} a}{c_{-} f} \frac{m_{e q}}{C Z}} \\
\text { Tangential Mode: } K_{\mathrm{T}}=8 R_{1} \mathrm{G}_{\mathrm{eq}} \text { con_l } G_{\mathrm{T}}=\sqrt{8 \mathrm{R}_{\mathrm{eq}} \mathrm{G}_{\mathrm{eq}} c_{-} l} \\
\text { Twisting Mode: } K_{\mathrm{TW}}=\frac{1}{2} K_{\mathrm{T}} c_{-} l^{2} \quad G_{\mathrm{TW}}=\frac{1}{2} G_{\mathrm{T}} c_{-} l^{2} \\
\text { Rolling Mode: } K_{\mathrm{R} 0}=\mathrm{R}_{\mathrm{eq}} K_{\mathrm{T}} \quad G_{\mathrm{RO}}=R_{e q} G_{\mathrm{T}}
\end{gathered}
$$

Finally, following [17] and [18], the net relative tangential, twisting, and rotating displacements between the two particles or objects over a given collision are defined in the following integrals where $t_{0}$ is the time at the start of the collision and $t$ is the current time.

$$
\bar{C}_{T}=\int_{t_{o}}^{t} \bar{v}_{T} K_{\mathrm{T}} d t
$$




$$
\begin{aligned}
\bar{C}_{T W} & =\int_{t_{o}}^{t} \bar{v}_{T W} K_{\mathrm{TW}} d t \\
\bar{C}_{R O} & =\int_{t_{o}}^{t} \bar{v}_{R O} K_{\mathrm{RO}} d t
\end{aligned}
$$

Using the vector and scalar parameters defined, expressions for the forces acting on the colliding objects can be formulated. The expressions provided are the forces and moments acting on particle 1. Oppositely directed forces and moments must be assigned to particle 2. In the normal direction:

$$
\bar{F}_{n}=-\left(\mathrm{K}_{\mathrm{N}}+G_{\mathrm{N}}\left(\bar{n} \cdot \bar{v}_{R}\right)\right) \bar{n}
$$

The force acting in the tangential direction is given by:

$$
\bar{F}_{\mathrm{t}}=-\left(\bar{C}_{T}+G_{\mathrm{T}} \bar{v}_{T}\right)
$$

If the magnitude of $\bar{F}_{\mathrm{t}}$ is greater than the critical force, $\mu\left|\bar{F}_{n}\right|$, then the force above is replaced by:

$$
\begin{gathered}
\bar{F}_{\mathrm{t}}=-\left(\mu\left|\bar{F}_{n}\right|\right) \bar{t}_{\text {tang }} \text { if } \bar{v}_{t} \cdot \bar{t}_{\text {tang }}>0 \\
\bar{F}_{\mathrm{t}}=\left(\mu\left|\bar{F}_{n}\right|\right) \bar{t}_{\text {tang }} \text { if } \bar{v}_{t} \cdot \bar{t}_{\text {tang }}<0
\end{gathered}
$$

The influence of the damping effect can be clearly seen in the two particle collision case in figure 5 . The pressure field that develops post collision with the damping is less vigorous and the particles are slower to separate as a portion of the initial kinetic energy of the particles is dissipated.

The twisting contact moment is defined in equation 31. This is a mainly three dimensional effect that is not explicitly observable in the studies conducted in the current work:

$$
\bar{M}_{T W}=-\left(\bar{C}_{T W}+G_{T W} \bar{v}_{T W}\right)
$$

If the magnitude of $\bar{M}_{T W}$ is greater than the critical moment, $\left(\frac{3 \pi}{16}\right)\left(\frac{c_{-} l}{2}\right) \mu\left|\bar{F}_{n}\right|$, then the moment above is replaced by:

$$
\begin{gathered}
\bar{M}_{T W}=-\left(\left(\frac{3 \pi}{16}\right)\left(\frac{c_{-} l}{2}\right) \mu\left|\bar{F}_{n}\right|\right) \bar{t}_{T W} \text { if } \bar{v}_{T W} \cdot \bar{t}_{T W}>0 \\
\bar{M}_{T W}=\left(\left(\frac{3 \pi}{16}\right)\left(\frac{c_{-} l}{2}\right) \mu\left|\bar{F}_{n}\right|\right) \bar{t}_{T W} \text { if } \bar{v}_{T W} \cdot \bar{t}_{T W}<0
\end{gathered}
$$

A rolling contact moment is also applied as in equations 33 and 34. This moment allows for the simulation of the rolling of a particle along a surface or rolling over another particle. The capability to include the process of a particle rolling over another is important in erosion and fouling effects and in the resuspension of particles into the flow. Without this feature, attempts to incorporate the rolling phenomena by the author were 
unsuccessful except when using a hard sphere collision model. A hard sphere modeling was not pursued further due particularly to the difficulties associated with simulating multiple simultaneous collisions. The rolling capability is illustrated in figure 6 , showing the rolling of two particles along a wall with the particle on the left initiating the rolling motion of the particle on the right.

$$
\bar{M}_{R O}=-\left(\bar{C}_{\mathrm{RO}}+G_{\mathrm{RO}} \bar{v}_{R O}\right)
$$

If the magnitude of $\bar{M}_{R O}$ is greater than the critical moment, $R_{1} \mu\left|\bar{F}_{n}\right|$, then the moment above is replaced by:

$$
\begin{aligned}
& \bar{M}_{R O}=-\left(R_{1} \mu\left|\bar{F}_{n}\right|\right) \bar{t}_{R O} \text { if } \bar{v}_{R O} \cdot \bar{t}_{R O}>0 \\
& \bar{M}_{R O}=\left(R_{1} \mu\left|\bar{F}_{n}\right|\right) \bar{t}_{R O} \text { if } \bar{v}_{R O} \cdot \bar{t}_{R O}<0
\end{aligned}
$$

When the particles are defined to be colliding, a cohesive force is activated between the particles or particles and walls. In the current model, the cohesive force was assumed to be a small fraction, $\beta$, of the normal force in equation 28 without dissipation. The cohesive force used in this work is given in equation (35) as from [17] with a value of $\beta$ of $1.20 \times 10^{-3}$.

$$
\bar{F}_{c o h}=\beta \mathrm{E}_{\mathrm{eq}} \frac{c_{-} a}{c_{-} f} \bar{n}
$$

Any customized cohesion force function can be incorporated into the model. The effects of the new cohesive force capabilities are displayed in figures 7 and 8 . Figure 7 shows a single particle interacting with a roughness element on a wall, with the cohesion force causing the particle to stick to the roughness element. Figure 8 illustrates the effects of varying the strength of the cohesion force, with stronger cohesion leading to particle agglomeration /fouling along the flow path surfaces, particularly the lower surface with roughness elements. Hence, the new capability facilitates a more detailed analysis of the particle flow conditions and improves the current state of the art.

Modifications to the formulations are required when the particles are contacting a wall. Further details can be found in [10-12,17 and 18].The force/moment formulations above must be applied to particle $\mathrm{P}_{1}$, with oppositely directly forces and moments acting on the second particle $\mathrm{P}_{2}$.

\subsubsection{Particle motion}

Based on the particle interaction, flow interaction, gravity interaction, and any other force effects, the translational and rotational motion of each particle can be determined. First, the translational motion of the particles is calculated. Under the stated loading, the acceleration of a particle o with mass $\mathrm{m}_{\mathrm{o}}$ is $\dot{\bar{V}}_{\mathrm{O}}$.

$$
\dot{\bar{V}}_{o}=\frac{1}{m_{o}}\left(\bar{F}_{n}+\bar{F}_{t}+\bar{F}_{\text {fluid }}+\bar{F}_{\text {grav }}+\bar{F}_{\text {coh }}+\bar{F}_{\text {other }}\right)
$$


The velocity of the particle at any time can be found by integrating the acceleration once with respect to time. The displacement can be found by integrating again with respect to time.

Next, the rotational motion of the particle is calculated. Due to potential issues with the use of standard Euler angles, a quaternion based formulation is implemented. The angular acceleration of particle o, ${ }_{\mathrm{B}}^{\mathrm{B}}{ }_{o}$, with a particle moment of inertia ${ }^{\mathrm{B}} \mathbf{I}$ is formulated with respect to its local body coordinate system, B, as in equation 37. The moment expressions within the equation must be transformed from the global coordinate system, $\mathrm{G}$, in the formulations provided above to the local body coordinate system, B.

$$
{ }_{\mathrm{B}} \dot{\bar{\Omega}}_{o}={ }^{\mathrm{B}} \mathbf{I}^{-1}\left({ }^{\mathrm{B}} \bar{M}_{n}+{ }^{\mathrm{B}} \bar{M}_{t}+{ }^{\mathrm{B}} \bar{M}_{T W}+{ }^{\mathrm{B}} \bar{M}_{R o}+{ }^{\mathrm{B}} \bar{M}_{f l u i d}+{ }^{\mathrm{B}} \bar{M}_{c o h}+{ }^{\mathrm{B}} \bar{M}_{\text {other }}-{ }^{\mathrm{B}} \bar{\Omega}_{o} \times\left({ }^{\mathrm{B}} \mathrm{I}^{\mathrm{B}} \bar{\Omega}_{o}\right)\right)
$$

The angular velocity is found by integrating the acceleration with respect to time once and the displacement from integrating the velocity with respect to time. Once the angular acceleration, velocity and displacement are determined in the body coordinate system, these values are transformed to the global coordinate system. Further details can be found in Shames [19], Ickes [20], and in the Appendix.

Information, in global coordinates, for the translational and rotational motion of each particle or other moving object is then returned to the computational fluid dynamics model and the objects are placed at their new position in the computational field and are supplied with the appropriate velocity. Then, the resulting flow, temperature, and pressure fields in the gas/fluid can be determined.

\subsection{Erosion rate model}

The final component of the model is responsible for estimating the erosion distribution along a surface that results from particle impacts. In the current method, an empirical based erosion rate model for sand particles impacting an aluminum surface is implemented as provided by Okita et al. [21]. With each collision between a particle and a wall, the Tulsa erosion ratio formulation estimates the mass loss due to the individual particle impact as in Eq. 36. This erosion ratio, ER, the ratio of the mass loss to the total particle mass, is given by:

$$
\begin{gathered}
E R=F_{S} C(B H)^{-0.59} V^{n} F(\theta) \text { with } \\
B H=\frac{\left(H_{V}+0.1023\right)}{0.0108}
\end{gathered}
$$

$\mathrm{V}$ is the magnitude of the particle velocity and the angle $\theta$ is defined as shown in figure $9 . F(\theta)$ is a parameter that varies with the impact angle, $\theta$, indicating the dependence of the rate of erosion or mass loss at impact to the angle at which the particle collides with the surface. The constant parameters for the case of the $300 \mu \mathrm{m}$ sand particle size impacting aluminum are given in table 1 . BH is the Brinell hardness of the normal plate, $\mathrm{H}_{\mathrm{v}}$ is the Vicker's hardness of the normal plate, $\mathrm{F}_{\mathrm{S}}$ is a sharpness factor for the particle, $\mathrm{f}$ is a normalizing factor, 
and $\mathrm{C}, \mathrm{n}_{1}, \mathrm{n}_{2}$, and $\mathrm{n}_{3}$ are empirical constants. The erosion rate, $\dot{e}$, in $\mathrm{kg} / \mathrm{m}^{2}-\mathrm{s}$, is then determined from equation 41 , where $\mathrm{R}$ is the radius of the particle impacting the surface, $\rho$ is the density of the particle material, and $\mathrm{A}_{\text {cell }}$ is the area of the computational element or cell upon which the particle has collided. $\dot{M}_{P}$ is essentially a "mass flow rate" of the particle material during impact.

$$
\dot{e}=E R\left(\frac{\dot{M}_{P}}{A_{\text {cell }}}\right) \text { with } \dot{M}_{P}=\rho V\left(\pi R^{2}\right)
$$

The erosion rate from each individual contact at a given computational cell element is then accumulated over the course of the simulation for the resulting total or net erosion rate distribution.

\subsection{Systems investigated}

In the development and investigation of the use of the direct CFD-DEM type coupling erosion rate prediction method, three main simulation study sets were conducted for the two system sets in figures 1 and 2 . The systems used for model validation and studies are described.

\subsection{Particles impacting a normal wall, effect of particle size}

The first system considered involves particles impacting a fixed plate at a 90 degree orientation to the initial particle motion direction as in figure 1a. The intent of the study is to investigate the effect of altered particle size, but with an equivalent net initial particle momentum, on the erosion that develops along this normal surface as a result of the particle impact. Three different particle sizes were selected, with the particle count varied so that the net particle mass is the same. The use of the same total particle mass for different particle sizes provides an equitable comparison of the erosion that will develop along a surface due to the same initial particle momentum. The three particle cases are: 24 particles at 1.01mm diameter (figure 1b), 68 particles at $0.60 \mathrm{~mm}$ diameter (figure 1c), and 272 particles at $0.30 \mathrm{~mm}$ diameter (figure 1d). In each case, the particles have the properties of silica or sand and the particles remain rigid in the computational domain. The flow path has the dimensions indicated in figure 1a with $\mathrm{H} 1=8 \mathrm{~mm}, \mathrm{~L} 1=95 \mathrm{~mm}$, and $\mathrm{L} 2=12.7 \mathrm{~mm}$, so that the end of the flow path is placed $12.7 \mathrm{~mm}$ from the plate surface. In each case, the tank zone configuration that feeds the flow into the flow path of interest, indicated in the red shaded area in figure 1a is the same. The fluid in the tank is initially prescribed a pressure of $100 \mathrm{MPa}$ and a temperature of $1700 \mathrm{~K}$ and is released at time $=0 \mathrm{~s}$, sending gas flow towards the particles positioned in the flow path. At 2.50e-05 seconds, the particles are given an initial velocity of $200 \mathrm{~m} / \mathrm{s}$, so that the net particle momentum moving into the wall is the same across all three cases, but the distribution of the particle momentum is different. This initial imparted velocity, the resulting particle interactions, and the flow induced forces as the high pressure and temperature gases pass over the particles govern the motion of the particles. The particle motion is traced, and as the particles impact the wall, the erosion rate model is implemented to estimate the mass loss rate distribution along the wall surface. Changes in the particle impact velocities, orientations, and positioning that develop for the different cases affect the resulting erosion at the wall surface. These differences and their origins can be analyzed using 
the simulation results. This same system configuration for 272 particles of $300 \mu \mathrm{m}$ diameter is used in the comparison case to the wall erosion results reported in Okita et al. [21].

\subsection{Particles impacting a normal wall, effect of impact velocity}

In the next set of studies, the 272 particle arrangement in figure $1 \mathrm{~d}$ is utilized, now with the initial particle velocity varied so that the effect of the particle impact velocity (momentum and kinetic energy) on the erosion distribution can be investigated for the same particle size. Particles are released inside of the flow tube at $2.50 \mathrm{e}-05$ seconds at speeds of 10,25 , and $250 \mathrm{~m} / \mathrm{s}$ with the same initial conditions assigned at the upstream tank as in the previous study. However, since the flow arrives at the particles at different times depending on the motion of the particles, the flow induced forces and the duration over which these forces act will vary. In fact, the slower initial particle speed of $10 \mathrm{~m} / \mathrm{s}$ case attains an average impact velocity of $620 \mathrm{~m} / \mathrm{s}$ since the particles experience greater flow induced forces for a longer period of time, while the $25 \mathrm{~m} / \mathrm{s}$ initial velocity case attains an average impact velocity of $530 \mathrm{~m} / \mathrm{s}$, and the $250 \mathrm{~m} / \mathrm{s}$ initial velocity case reaches an average impact velocity of $430 \mathrm{~m} / \mathrm{s}$. The erosion magnitudes and distributions can then be analyzed as a function of the average impact velocity.

\subsection{Particle motion and erosion for three configurations}

In the last set of studies, the three configurations of increasing complexity shown in figure 2 are used to examine the motion of the $272300 \mu \mathrm{m}$ diameter particles as they proceed along the three flow path geometries, collide with the walls, and cause particle impact based erosion. These geometric configuration types of a diverging/converging section (figure 2a), a by-pass flow path (figure $2 b$ ), and a two baffle configuration (figure 2c) are common in industrial systems. Since the flow in these geometries is forced to change direction, the configurations promote particle impact with the flow path surfaces, and the resulting erosion can be investigated. Hence, the application of the modeling method to these configurations provides information on the utility of the techniques devised to study the erosion distributions in such systems. The insight and information gained can then can be used to suggest alterations to the system design to modify the erosion patterns as desired. With the same initial conditions applied to the "tank" zone in the two study sets, the tank and flow path geometries and conditions leading to the new geometric configurations are the same as those from the previous cases in figure 1 . The particles are released with a velocity of $200 \mathrm{~m} / \mathrm{s}$ at $2.50 \mathrm{e}-05$ seconds. In the diverging/converging configuration in figure $2 \mathrm{a}, \mathrm{H} 1$ is $8 \mathrm{~mm}$ and $\mathrm{L} 1, \mathrm{~L} 2$, and $\mathrm{L} 3$ are all equal to $12.7 \mathrm{~mm}$. In the by-pass configuration in figure $2 \mathrm{~b}, \mathrm{H} 1$ is $10 \mathrm{~mm}, \mathrm{H} 2$ is $4 \mathrm{~mm}, \mathrm{~L} 1$ is $4 \mathrm{~mm}, \mathrm{~L} 2$ is $28 \mathrm{~mm}$, and $\mathrm{L} 3$ is $4 \mathrm{~mm}$. Finally, in the baffle configuration in figure 2c, both baffles have the same dimensions, with L1 equal to $8 \mathrm{~mm}$, $\mathrm{H} 1$ equal to $8 \mathrm{~mm}$, and $\mathrm{H} 2$ equal to $7.5 \mathrm{~mm}$. The portions of the system surfaces most prone to erosion can be identified from the results of the model. 


\subsection{Numerical specifications}

The modeling technique utilizes the solvers in the finite volume based code FLUENT(), Release 16, coupled with customized author written code. In the investigations, the finite volume based solution methods are used to solve the governing equations in the fluid, given in equations 1 through 4 , with the appropriate modifications employed for remeshing and the moving cell elements. Basic finite volume discretization was developed by Patankar [22]. An ideal gas equation of state is implemented for the air, the compressible gas in the study, with temperature varying specific heat, thermal conductivity, and viscosity. An explicit solution procedure is used in space with second order upwinding and Green-Gauss node based gradient discretization, with AUSM flux type formulation, which was developed for improvements in the ability to capture of pressure wave propagation. First order discretization in time is used in the current model.

\subsection{Boundary conditions}

All walls in the system are assigned no slip type boundary conditions so that the fluid is fixed to the nonmoving walls and moves at the same speed as any of the moving particle surfaces. Solid boundaries are also assigned insulated thermal boundary conditions so that no heat flow from the fluid to the solid bodies or between solid bodies is taken into account in the current formulation. The outlet boundary of the chamber, by-pass, and baffle geometries in figure 2 are assigned pressure outlet boundary conditions with an ambient pressure of 101,325 Pa.

\subsection{Initial conditions, time step}

The gas is initially assigned ambient temperature and pressure conditions, with no flow velocity. For the $\kappa-\varepsilon$ realizable turbulence model, initial turbulent kinetic energy of $0.01 \mathrm{~m}^{2} / \mathrm{s}^{2}$ and turbulent dissipation rate of 100 $\mathrm{m}^{2} / \mathrm{s}^{3}$ are assigned. At 2.0e-05 seconds from the start of the simulation, which is sufficient for the particles to come to an equilibrium position under the contact/cohesion forces, the particles are given an initial velocity. A time step of 5.0e-09 seconds was selected with 20 iterations per timestep.

\subsection{Computational resources and mesh sensitivity}

Between 32 and 64 processors were used in the calculations, pending availability on various Linix based clusters, with run times ranging from three days to a week depending on the model/system investigated.

As in figure 3, the particles/particle surfaces are discretized within the fluid computational domain. The mesh around the particle surfaces must be sufficiently fine to properly resolve the fluid induced forces. A thin finer mesh moves with the particles to assist in resolving the boundary layer flow. A sample of the mesh with the boundary layer around the moving particles is shown in figure $3 \mathrm{~b}$. The final mesh size used in this study involves a 4.0e-05 m element size with triangular elements as required by the remeshing/mesh motion algorithm of FLUENT $\odot$. This was found to be approximately 24 elements around the circumference of the smallest particle size considered. Sensitivity studies were conducted for the 272 particle $300 \mu \mathrm{m}$ particle diameter case, as the flow and mesh resolution at this minimum particle size studied is then appropriate for larger size particles. Impact speeds of one specific particle were within 3\% of those for a mesh with a 16 
element count around the circumference and the net erosion rate at the plate surface was within $2 \%$. The mesh with the $4.0 \mathrm{e}-05 \mathrm{~m}$ size was therefore selected for the investigation.

\subsection{Model comparison to published results}

The results for the case of $272300 \mu \mathrm{m}$ particles impacting the normal wall in figure $1 \mathrm{~d}$ were compared to the empirical based data published by Okita et al. [22]. For the case of an impact velocity of $25 \mathrm{~m} / \mathrm{s}$ or a $\mathrm{Re}_{\mathrm{p}}(\mathrm{VD} / \mathrm{v}$ ) of approximately 500, the erosion ratio (ER) reported by Okita was $3.5 \mathrm{e}-05$ compared to $2.92 \mathrm{e}-05$ as predicted in the current work, and for an impact velocity of 50 $\mathrm{m} / \mathrm{s}$, or a $\mathrm{Re}_{\mathrm{p}}$ of approximately $1000,1.1 \mathrm{e}-04$ compared to $9.71 \mathrm{e}-05$ in the current work. Hence, the current model well replicates the erosion conditions that develop. The erosion due to impact is only calculated once per contact in the current model. Rolling particles or particles that release slightly and then return to impact the surface due to interactions with other particles and with the flow are not accounted for in the erosion effects predicted. This could cause the slightly lower erosion rates predicted by the model when compared to the previously published empirical data.

\subsection{Results}

For the three major sets of numerical studies conducted, the particle motion patterns and the resulting particle impact based erosion predicted by the model are described and discussed below. Animations of velocity, pressure, and temperature fields of all of the studies conducted are available from the author.

\subsection{Particles impacting a normal wall, effect of particle size}

In the first study, the effect of particle size on the particle motion and erosion for the same net initial particle momentum is investigated using the system in figure 1a. Three different particle configurations in figures $1 \mathrm{~b}$, 1c, and 1d provide the same net particle mass and same net initial particle momentum with the gas flow released from the tank proceeding in the same manner. However, the size and mass of each individual particle is different across these three cases, producing an altered initial particle geometric and momentum distribution. With different initial gap spaces between the particles and between particles and the wall, the initial and subsequent flow moving over the particles vary with particle size. Further dissimilarities in the particle and flow conditions develop as the heavier particles require greater force/momentum change to be accelerated at the same rate as the lighter (smaller) particles. Hence, the particle motion is interdependent with the particle size, the particle mass, the time varying proximity to other particles and to the walls, the local gas flow conditions that result, and any collisions with other particles and the walls of the flow path during the history of the particle travel. Since the particle impact based erosion is a function of the particle impact velocity and orientation along with the particle mass, the factors affecting the particle motion will also influence the particle based erosion distributions that result. 
The characteristics of the particle flow clearly vary with the particle size for the three cases studied. Figures 10, 11, and 12 depict contour plots of the gas velocity and gas pressure at four times during the simulation to illustrate the features of the particle motion and relationships between the particle motion and the gas flow and geometric conditions. The view in figure 12 is slightly different than for the other two cases to allow for better visualization of the particle conditions. Even at the start of the simulation, differences in the particle motion can be observed for the three cases comparing figures 10a, 11a, and 12a. The particles of the larger size reach the end of the flow path at a later time for the conditions considered, since the smaller and lighter particles achieve a higher acceleration and faster velocity as imparted by the fluid induced forces. The lower velocities in the gas in the regions just outside the particle pack for the smaller particle size in figure 12a compared to those in figure 10a and the higher pressures acting on the larger particles in figure 10a are consistent with the faster moving smaller particles.

The smaller particles are more susceptible to particle spread as the particles exit into the surroundings. The lower particle mass means the particles are more readily accelerated in the direction normal to their original motion. This effect can be observed in figures $10 \mathrm{~b}, 11 \mathrm{~b}$, and $12 \mathrm{~b}$, where, as the particles exit into the surroundings, the relative motion of the external rows of particles on the top and bottom layers of the particle pack with respect to their original conditions becomes more significant as the particle size decreases. As the particle relative position shifts, flow paths open, instigating further spread. The more extensive pressure differences across the particles for the smaller particle size can be seen in these figures. The different geometric arrangement of the particles with the smaller particle size contributes highly to the differences in the gas flow through the smaller particle size configurations and the resulting differences in the flow conditions acting on the particles. This trend is maintained as the particles move towards the wall so that the overall spread in the particle distribution is greater for the smaller particle size just before the particles impact the normal plate. The particle motion and the gas flow from the tank contribute to the increase in the pressures and temperatures at the wall surface, with a pressure wave reflected from the wall towards the flow channel prior to impact, potentially affecting the particle motion. The resulting high pressure area appears to be larger for the smaller particle size case. The good resolution of the pressure reflections from the surfaces of the flow channel can also be observed in the results as seen by the diagonal demarcations in the pressure contours near the flow path exit.

The particles then impact upon the normal surface. In addition to the local flow conditions resulting from the gases exiting the flow path and moving over the particles, the flow generated by the moving particles, the flow reflecting from the wall, and the collisions of the particle with the wall and other particles direct the particle motion. During this time the particle-particle interactions are particularly significant in determining the shape and distribution of the particle arrangement as the particles impact upon the wall and, therefore, in forming the erosion patterns that occur as a result of these particle impacts. 
The particle arrangements just after collision and the velocity and pressure contours in figures 10c, $11 \mathrm{c}$, and $12 \mathrm{c}$ clearly demonstrate the differences in the distribution or arrangement of the particles as they interact with the wall surface and the differences in the size of the region along the normal plate surface affected by the particles for the three particle size cases. As the particle size decreases the particle arrangement takes on a more fluid like appearance, with a parabolic-like pile of particles resembling a liquid droplet as in figure 12c. In contrast, for the larger particle size, the particle - particle interaction is less frequent as the particles move apart more quickly after contact with the wall, perhaps due to the increased momentum transfer during collision with the wall for these larger and heavier particles and the lower particle count as in figure 12a. Clearly, the zone along the plate affected by the particle flow and erosion is larger for the smaller particle size.

As the particles continue to interact with the fixed wall and each other, and the flow, these characteristics persist. For the largest particle size case, in figure 10d, the main contact between the particles and the wall occurs with the particles at the front of the particle pack, with the remaining particles rebounding off of these "front" particles as the front particles return from collision with the wall surface. While the larger particles may have more momentum due to their larger mass, the frequency of the collisions with the wall and with other particles is reduced due to both the smaller particle count and the nature of the particle distribution and particle-wall interactions. Hence, the local erosion rates are lower (Figure 13). Figure 12d clearly illustrates the greater portion of the length of the normal plate upon which particle-wall interaction has occurred for the smaller particle size. Therefore, the area over which erosion due to particle impact develops is also more extensive (figure 13). Hence, the distribution of the erosion as well as its magnitude are affected by the particle size. While the particle mass/momentum is lower for the smaller particle size cases, the frequency of the collisions and extent of the eroded area increase with smaller particle sizes. Therefore, an intermediary particle size, where the higher particle momentum combines with a sufficiently high collision frequency, is most likely to produce higher erosion rates.

The erosion distribution at the conclusion of each of the simulations is provided in figure 13 . The 24 larger particle size case attains a parabolic like distribution with most erosion developing opposite the flow path centerline. The erosion results mainly from the impact of the first column of particles in the initial configuration with little spread or deviation in the particle path from the time period just after the particles move into the surroundings. Though the particles are of the largest size, the configuration does not produce the highest local or net magnitude of the erosion due to the reduced frequency of particle impact. The case also produces the most compact of the erosion distributions presented. The mid-size 68 particle arrangement produces the largest local magnitude and net value of the erosion rate. The local peak occurs in the same zone as the 24 larger diameter particle case, near the flow-path centerline. Slight oscillations in the distribution to the sides of the peak erosion rate result from the particle-particle interactions and the particle-flow interactions that have spread over a larger area. However, a significant change in the distribution shape is noted for the 272 
smaller particle configuration. The erosion rates are lower to the center of the plate and then increase in the areas just off center before falling off. With the smaller particle size, the particles act more fluid-like. The frequency and speed of the particle interactions are lower in the stagnant zone opposite the centerline of the exiting flow path and higher gas flow/particle flow velocities are found just outside of this stagnant zone. Such a distribution is also described by Gnanavelu et al. [23], who depicted a similar wear pattern to that found in figure 7 for the 272 small size particle configuration. Hence, the particle size and initial positioning distribution, even with the same net initial particle momentum, can significantly alter the erosion rate distribution along the plate as well as the erosion magnitudes.

The results of this study indicate as the particle size decreases, the particle motion begins to replicate the characteristics of a fluid with particle-particle interactions playing a significant role in the distribution of the particles as they impact the normal wall and therefore the ensuing erosion. The larger particle sizes tend to produce narrower zones of erosion since the particle spread before impact is low and the particles tend to rebound from the wall swiftly and with sufficient magnitude to dissuade the next incoming particles from impacting the surface. Intermediate particle sizes are more likely to produce higher local erosion rates since they may maintain sufficient momentum to cause material loss from the surface, but also allow for higher particle wall contact frequency due to the higher particle counts as well as the increased interaction between the particles. Particle motion and erosion characteristics shift with the smaller particle size as the wear pattern showed a lower wear rate zone near the centerline of the flow channel/plate and higher erosion to the sides, following a distribution more like the velocity of a fluid impacting a plate.

\subsection{Particles impacting a normal wall, effect of particle impact velocity}

In the second study conducted, the system in figure $1 \mathrm{~d}$, with $272300 \mu \mathrm{m}$ diameter particles, is utilized to investigate the effect that the impact velocity has on the erosion distribution that develops at the normal wall. Hence, the initial particle momentum and kinetic energy is varied for the same particle size. While the particles are all released at a time of 2.5e-05 seconds, three initial velocity cases are studied with velocities of 10,25 , and $250 \mathrm{~m} / \mathrm{s}$. Thus, the time at which the gas flow from the tank area reaches the particles will vary for each case since the particles are moving along the flow path at different rates. In fact, the initially faster moving particles released at $250 \mathrm{~m} / \mathrm{s}$ have cleared the flow path before the high pressure gas wave passes over the particles, while the particles remain within the flow path as the high pressure gas flow stream passes over the particles for the two slower initial velocities. As a result, a significant difference in the fluid induced forces acting on the particles occurs. The fluid force induced acceleration of the particles in the direction of the flow path is much higher for the slower particles as they not only experience higher pressure level gases, but also experience these flow induced forces for a longer period of time before the particle pack moves into the surroundings and the gas flow expands. By the time the particles impact the normal wall, the initial $10 \mathrm{~m} / \mathrm{s}$ particle release case produces an average impact velocity of $620 \mathrm{~m} / \mathrm{s}$, the $25 \mathrm{~m} / \mathrm{s}$ case gives an average impact 
velocity of $530 \mathrm{~m} / \mathrm{s}$ and the $250 \mathrm{~m} / \mathrm{s}$ case yields an average impact velocity $430 \mathrm{~m} / \mathrm{s}$. Hence, the impact velocity ranking is the reverse order of the ranking of the initial velocity imparted to the particles.

The initial contact of the high speed, high pressure tank flow with the particle surfaces occurs with particles positioned further along the flow path or into the surroundings as the initial particle speed increases. These conditions are apparent comparing the velocity and pressure contours in figure 14 a for the $250 \mathrm{~m} / \mathrm{s}$ initial velocity case, figure $15 \mathrm{a}$ for the $25 \mathrm{~m} / \mathrm{s}$ initial velocity, and figure $16 \mathrm{a}$ for the $10 \mathrm{~m} / \mathrm{s}$ initial velocity. The higher pressures acting on the particles and the greater extent of the high pressure region, particularly to the rear of the particle pack, for the slower initial particle velocities at the time the gas flow first reaches the particles can be readily observed in these plots. The stronger fluid induced forces accelerate the particles in a direction mainly along the flow path. However, the higher forces acting on the particles to the rear of the pack result in particle - particle as well as particle-flow path wall interactions. These interactions alter the particle arrangement into a more random distribution, especially in the rear region (figure 16a), when compared to the orderly rows nearly maintained by the $250 \mathrm{~m} / \mathrm{s}$ initial velocity case which experiences little effect of the tank flow prior to exit from the flow path (figure 14a). As the particles move from their orderly arrangement for the lower initial velocity/higher impact velocity cases, the new particle positioning for the slower initial particle speeds more readily allows for gas to move between the particles, inciting further interactions. The inset in figure 16 a for the $10 \mathrm{~m} / \mathrm{s}$ initial case clearly shows the modified particle arrangement as the particles move towards the exit of the flow path.

The more vigorous particle interactions within the channel for the slower initial particle speed cases lead to a more disperse particle distribution in the surroundings as the particles leave the flow channel, seen in comparison to the high initial speed case (figures $14 \mathrm{~b}, 15 \mathrm{~b}$, and 16b). This more distributed particle arrangement affects not only the particle interactions that develop, but also the flow conditions. With the larger spacing between the particles, stronger and more extensive flow streams move between the particles, leading to greater imbalances in the pressure forces acting on the particles and further relative motion of the particles. These flow-induced forces continue to alter the trajectory of the particles. As the relative particle positioning shifts in response, the flow over neighboring particles is altered and particle - particle interactions may develop or be diverted. As a result of the significantly altered particle arrangements from the initial configuration for the slower initial particle velocity cases, the spread or distribution of the particles across the domain is larger than for the faster initial particle velocity cases. In the faster initial particle motion case (figure 14b), the particles remain in rows, excluding the exterior rows (top and bottom) that begin to peel away from the particle pack. The pressure wave reflections from the flow path walls, the diagonal demarcations within the flow channel in the pressure contour plots, can be observed most clearly and consistently for the higher particle initial velocity case in which the particles have cleared the flow path sooner. The well resolved reflections are more rapidly dissipated by the presence of the particles in the flow channel and also by the flow reflections from the normal (impacted) wall that eventually develop and reach the flow path entrance. 
Another difference in the characteristics of the particle flow exiting from the flow channel is the more asymmetric nature of the particle positioning and, therefore, the flow patterns just after particle exit, as the initial particle velocity decreases as seen in figures $14 \mathrm{~b}, 15 \mathrm{~b}$, and $16 \mathrm{~b}$. Such patterns may be attributed to the specific particle-particle, particle - flow, and particle-wall interactions and the longer acting gravitational forces directed from the top surface of the flow channel downward to the bottom surface during the time over which the particles are in the channel. For the lowest initial velocity case, two or three particles appear to "stick" to the lower surface of the flow path until the fluid forces overcome the adhesive forces, noticeable in figure 16b. Hence, particles in the zone above the channel centerline tend to attain higher velocities and move further along or exit from the flow channel than those in the area below the channel centerline at the same period of time. These conditions could not be observed without the inclusion of the cohesion forces and can significantly affect the particle motion behavior within the flow channel and as the particles move into the surroundings.

While the higher initial particle speed case maintains a more symmetric particle and flow distribution as the particles move across the gap between the end of the flow path and the normal plate, the asymmetric nature of the particle and flow conditions for the two slower initial particle velocities persists as the particles approach the normally oriented plate. A symmetric gas pressure build-up develops at the normal surface for the $250 \mathrm{~m} / \mathrm{s}$ initial particle velocity case, and the particles keep nearly the same relative positioning, excluding the upper and lower particle rows which have peeled away from the particle pack from back to front. However, the particle distribution maintains its symmetric nature (figure 14c). In contrast, with the two slower initial particle velocity cases, though the initial gas flow impacting the normal surface resulting from the tank flow alone is symmetric (figure $15 \mathrm{~b}$ and figure $16 \mathrm{~b}$ ), the asymmetric particle distribution results in more frequent particle impacts on the upper portions of the plate surface than on the lower (figure 15c and figure 16c) during the time of initial particle impact. Additionally, the gas pressures along the wall near these colliding particles increase due to the presence of the moving particles and the flow induced by the particle motion (figure $15 \mathrm{c}$ and figure 16c), perhaps further contributing to the initially asymmetric erosion distribution.

These trends in the particle distributions remain even just after particle collision with the wall occurs, as shown in figures $14 \mathrm{~d}, 15 \mathrm{~d}$, and $16 \mathrm{~d}$. With the more frequent interactions with the upper portions of the wall for the lower initial speed cases, more intense erosion initially develops along the upper portions of the normal surface as compared to the lower. The higher erosion levels in the upper portion of the normal plate are apparent for the $10 \mathrm{~m} / \mathrm{s}$ and $25 \mathrm{~m} / \mathrm{s}$ initial velocity cases in figure 17 , though these plots show the erosion at the completion of the simulation. In comparison, for the $250 \mathrm{~m} / \mathrm{s}$ case, though the particle spread increases as the pack moves from the flow channel to the wall and more rows show changes in relative particle positioning, the particle distribution pattern and thus the collision and resulting erosion distribution patterns at this initial collision stage are symmetric. 
At later stages of the collision process, the trends in the particle motion are altered. As the particles impact the normal surface, the particles to the rear continue to move towards the normal plate with those ahead forced into contact with the wall or other particles. The particles to the rear of the pack either impact the normal plate or interact with particles already ahead, particles that may be rebounding or rolling along the normal surface. As a result of this forced particle interaction, the distribution of the particle arrangement becomes more symmetric even for the slower initial particle velocity cases, which now have the faster swpeed at impact). The particle distributions in figures $15 \mathrm{e}$ and $16 \mathrm{e}$ more closely resemble the distributions in figure 14e. With a parabolic like cluster of particles that flattens as the upper particles move or roll along the plate from the centerline upwards and the lower particles move or roll from the centerline downward. The erosion that was initially focused closer to the channel centerline area then continues to spread with time. As the particles move away from the centerline, however, the particle impact orientations tend to be more shallow and the impact velocities tend to be lower, leading to reduced erosion rates. As the erosion rates are cumulative and the initial local erosion rates are more intense, the initially asymmetric erosion conditions persist in the final erosion rate distributions for the two slower initial particle motion cases.

The cumulative erosion rates as a function of position along the normal plate illustrate the trends in the erosion patterns described above, and the final erosion rate distributions are shown in figure 17. As with the minimum particle size case in the particle size investigation in section 5.1, in all three initial velocity cases, the erosion is greatest, not at the centerline, but slightly off center, away from the particle and flow stagnation zone. Again, this trend is noted by Gnanavelu [23]. The erosion pattern for the initial $250 \mathrm{~m} / \mathrm{s}$ case is more symmetric, while the distribution is more asymmetric for the initial 10 and $25 \mathrm{~m} / \mathrm{s}$ cases, with higher erosion found in the upper regions of the normal plate. The asymmetric particle distribution as the particles first impact the plate for the initially slower velocity cases compared with the more symmetric arrangement for the initially higher velocity cases is consistent with these characteristics. While at later times the particle interaction forced by the presence of the plate and the particles results in a more symmetric particle distribution, because the erosion rate is cumulative and the initial erosion rates are higher, the net particle impact based erosion remains asymmetric for the conditions studied. The higher impact velocity also leads to higher local erosion rates. The net erosion rate over the entire plate surface is plotted in figure 18. The results indicate a nearly linear relationship between the average impact velocity and the net erosion rate, with the higher collision speeds creating greater mass loss from the plate surface. This trend is consistent with the erosion ratio results presented by Okita [21].

The model results have demonstrated that the erosion rates are a strong function of the impact velocity, with the higher impact speeds generally resulting in higher local erosion rate magnitudes and higher net erosion rates. However, the distribution and motion of the particles is affected by the conditions leading up to particle impact at the wall. 


\subsection{Particles motion and erosion in three system configurations}

In the final set of studies, the modeling techniques develops are used to study the motion of particles and the resulting particle impact based erosion for three system configurations that force particle-flow path interactions. These types of geometries are common in many industrial systems where erosion may be an issue to system performance. The three geometries are shown in figure 2: a diverging/converging section, a bypass flow path, and a two baffle arrangement. The same $272300 \mu \mathrm{m}$ particles as in the previous study were released at $2.50 \mathrm{e}-05$ seconds at a speed of $200 \mathrm{~m} / \mathrm{s}$ from the end of the flow path that leads into each of these specific geometries. Again, the flow path leading to the geometry is fed by a tank initially assigned the same elevated pressure and temperature as in section 5.2.

\subsubsection{Diverging/Converging section configuration}

For the geometric configuration in figure $2 \mathrm{a}$, a diverging/converging section, the modeling technique can assist in revealing the areas along the flow path surface most susceptible to particle impact based erosion. The particles are given an initial velocity along the flow channel and the tank gas reaches the particle pack, leading to a pressure rise in the gas to the rear of the particle pack as in figure 19a. The higher pressure gas conditions spread through the pack of particles over time and lead to the acceleration of the particles along the flow path. As the particles reach the end of the flow path, the gas is free to expand, and the gas velocity increases and pressure decreases. In the more open area outside of the flow path, the top and bottom rows of particles begin to peel away from the particle pack from back to front as a result of the fluid induced forces acting on the particles. Particle-flow and particle-particle interactions in the remainder of the particle pack lead to slight adjustments in the relative particle positioning (figure 19a). As the flow expands, the gas moves along the diverging section in a manner nearly parallel to the angled surface. While the majority of the particles proceed mainly in their original direction, as particle spread develops, some particles, mainly on the exterior of the particle pack, are forced by the fluid to follow trajectories that carry them nearly parallel to this diverging section surface. Any infrequent collisions/contact that develop occur at shallow angles near the end of the diverging section where the flow path shifts to a horizontal surface and the flow is forced to change direction. Therefore, the erosion rates along this diverging portion of the surface are lower. The erosion rates that develop along this surface, $\mathrm{S} 1$ in figure 2a and similarly along the upper diverging section. During this time, the first, most downstream column of particles begins to separate from the particle pack as a result of the fluid induced forces, with high pressures acting on the rear of the particles and the low pressure on the front particle surface, where the particles in the remaining columns experience elevated pressures around most of their surfaces.

The particles then begin to move through the straight section of the flow path, though the higher speed gas has already reached the converging section of the system and has begun to exit the converging section (figure 19b). Some pressure wave reflections from the converging section geometry are visible at this time. The change in the slope of the flow path at this horizontal section not only redirects the flow, but also redirects 
the particle motion. While the majority of the particles are not situated near the horizontal section of the wall, the particles that had moved along or near the diverging section do, at times, collide with the horizontal wall. The early collisions occurring near the start of this horizontal section have shallow impact angles as the particles move nearly parallel to the flow path. Figure $19 \mathrm{~b}$ depicts how a small portion of the total particles, largely the top and bottom rows and the first, most downstream, particle column have moved towards the horizontal walls. As the flow streams moving through the system are redirected by the converging portion of the flow path while the flow and particle motion continues, reflections from the converging surfaces develop. This change in the flow, along with the local particle-particle interactions, lead to more frequent collisions at the horizontal surface at higher velocities and with larger impact angles. As shown in figure 20a, the erosion on the S2 surface in figure 2a, first experiences higher magnitudes towards the downstream corner of the horizontal surface $(9.00 \mathrm{e}-05 \mathrm{~s}$ curve), and then this erosion spreads backward at times of $1.00 \mathrm{e}-04$ and $1.50 \mathrm{e}-$ $04 \mathrm{~s}$ as the gas flow and the particle-particle and particle-wall interactions not only redirect the gas flow but also redirect the particle motion to steeper impact angles, leading to higher erosion rates. Similar trends along the top horizontal surface are also observed.

While significant erosion is developing on the downstream end of the horizontal surfaces of the flow path section, the highest erosion rate magnitudes and most widespread erosion develop along the converging section of the flow path, surface S3 in figure 2a. The gas flow is constrained by the smaller flow path size, forcing the gas and the particles into this restricted flow area. The gas velocities decrease and the pressures and temperatures increase near the converging surfaces as the kinetic energy is converted to pressure and thermal energy. Particle-particle contact increases and the flow and particle interaction forces particle impact along the converging section surface, beginning with the downstream most end of the surface and progressing upstream as the pressure build-up and particle density increase as seen moving from the flow and particle conditions in figure $19 \mathrm{c}$ to those in figure 19d. The build-up of particles in the area that leads to the forced interaction and erosion is clear in figure 19d. The maximum erosion rates in the lower converging section as depicted in figure $20 \mathrm{c}$ are over two times those along the horizontal surface in figure $20 \mathrm{~b}$. The geometric arrangement and the resulting flow incites more frequent impacts, at steeper angles relative to the surface, and over an increasingly wide-spread portion of the converging section.

The overall erosion rates along the three lower portions of the flow channel are provided in figure 20c at the three times of $9.00 \mathrm{e}-05,1.00 \mathrm{e}-04$, and $1.50 \mathrm{e}-04$ seconds to show the development and accumulation of the erosion as a function of time and follow the trends and characteristics described. The converging section (S3) clearly experiences the highest erosion rates with the net erosion at $1.50 \mathrm{e}-05$ seconds over double that on the horizontal portion (S2) of the flow path. The erosion on this S3 surfaces develops later in time compared to the horizontal section since the passage of flow and particles is delayed. The modeling method clearly establishes that the downstream converging surface is most susceptible to erosion for the converging diverging configuration and depicts the conditions that lead to the susceptibility of certain areas to higher erosion. 


\subsubsection{By pass configuration}

In the second geometric configuration, the particle motion and erosion that develops in the bypass type arrangement in figure $2 b$ is simulated. Again, the particles are released from within the flow channel and they are then forced through the system which includes a by-pass alternative flow path, leading to a secondary flow path. For the current configuration and flow conditions, about $2 \%$ of the particles leave the main flow path and move into the by-pass section. To exit from the main flow path and move into the by-pass, the forces acting on a particle must be sufficient to alter the particle trajectory and the timing of these forces must allow for the momentum change to occur before the particle passes the by-pass section. The high pressure tank gas flow produces loading on the particles that is highest at the rear of the top row of particles and produces sufficient and timely forces that send a set of particles into the by-pass as shown in figure 21a. The particles immediately strike the right side surface of the by-pass flow path due to the flow and geometry in the system as shown in the inset plot in figure 21a. Figure 22a depicts the erosion rate along this right surface marked S6 in figure $2 b$, demonstrating the higher erosion rates at the entrance area of this flow surface. The particles then roll or bounce along the surface, and accelerate as a result of the high speed gas flow induced forces.

Nearly all of the particles that enter the by-pass proceed to impact the surface S9 in the area just opposite where the flow exiting the by-pass enters the secondary flow path, near the zone just below the S9 label, with the elevated surface pressure in figure 21a. Due to the system configuration, the impact angles for these particles are between 45 and 90 degrees (figure 21b). Together with the generally faster particle velocities, the higher impact angles produce greater wear or erosion of the solid material surfaces near this position along S9 when compared to the erosion that developed at the S6 surface from main flow path particle bleed-off at shallower particle impact angles and lower impact velocities. Figure $22 \mathrm{~b}$ shows the erosion rate development on the S9 surface with the surface direction defined pointing from the closed end on the right to the outlet on the left. The maximum local erosion rate is about three times higher than that on the by-pass flow surface S6 and the net (integrated) erosion rate on S9 is about two times greater than that on the S6 surface.

As the flow enters the secondary flow path, the highest flow velocities tend to be towards the left, towards the open end of the system along the secondary flow path, while the pressure builds in the area of the closed end of the system as in figure 21c. Within the low velocity, higher pressure circulation zone that develops at the closed end of the system, approximately $80 \%$ of the particles that move into the by-pass become trapped or circulate in this area as seen in figures $21 \mathrm{c}$ and $21 \mathrm{~d}$. With the reduced particle velocities in this circulation zone, lower levels of erosion develop on surfaces S7 and S8 as defined in figure 2b, than the erosion on the S9 surface. The orientation of the S7 surface which is nearly parallel to the flow circulation, combined with the slower impact velocities result in about 100 times lower peak erosion on the S7 surface as compared to the S8 surface which is oriented normal to the main secondary flow path direction. 
The net erosion rate results for this by-pass configuration, summarized in figure 22c, show the major erosion for the conditions studied occur along the right side surface of the by-pass flow path, S6, the closed end of the secondary flow path, S8, and the top surface of the secondary flow path, S9. These surfaces experience the more frequent particle impacts at higher speeds and at orientations that produce greater mass loss. Changes in the geometry can be suggested to alter the erosion patterns along the surfaces.

\subsubsection{Baffle configuration}

The final configuration investigated for areas prone to erosion is the baffle arrangement in figure $2 \mathrm{c}$, involving two equal sized baffle chambers. Similar to the two previous configurations, the particles are released into the flow channel upstream of the baffle area and high pressure and temperature gas flow is released from an upstream tank. The first particles that move into the first baffle volume as, shown in figure 23a, are those particles originating from the rear of the top and bottom rows of the particle pack, that are most affected by the gas flow and begin to spread from the particle pack. About eight percent of the particles move into the first baffle chamber. The entering particles impact surface S6 first (and similarly for the corresponding surface in the upper portion of the baffle configuration), initiating the particle based erosion along this surface. The erosion distribution along S6 at specified times are provided in figure $24 \mathrm{~b}$. As the particles and gas move into the first baffle chamber, a counterclockwise circulation develops in the upper baffle chamber zone and a clockwise circulation develops in the lower baffle chamber zone with the particles following with the flow and the baffle chamber surfaces in a similar pattern. The particles reach the top of the first baffle chamber and simultaneously, particles begin to move into the second baffle chamber as well (figure 23b). About 12 percent of the particles move into the second baffle chamber.

As the particles circulate around the baffle chambers, the particles in the first baffle chamber begin to impact the upper and lower baffle surfaces (surface S5 in the lower baffle section) as in figure 23c. The location of the most frequent and intense particle-lower baffle surface interaction occurs at the downstream, right side of the surface as the particles moving along the S6 surface are proceeding nearly perpendicular to the lower S5 surface. The interactions with the baffle surfaces and the flow reduce the kinetic energy of the particles, and the particle velocities decrease. After the initial contact with the horizontal first baffle chamber surface, near the right end of the surfaces, the particle-wall and particle-particle interactions as well as the flow conditions tend to produce particle trajectories that send the particles slightly away from the horizontal surfaces (S5), with the particles continuing in a general clockwise circulation loop in the lower portion of the first baffle (counter-clockwise in the upper). Particle progress is also slowed or hampered by adhesion forces between the particles and the baffle walls as seen in the upper right corner of the first upper baffle volume and lower right corner of the first lower baffle volume (figures $23 \mathrm{~b}, \mathrm{c}, \mathrm{d}$ ). These particle motion characteristics affect the erosion rates that develop. The higher erosion rates at the S5 surface, in figure 24a, are concentrated in a small portion of the near the downstream end. While the maximum local erosion at the S5 surface is about 
twenty-five percent higher than that at the S6 surface, due mainly to the different impact orientations, the net erosion at S5 is about one-half that at the S6 surface.

As the particles move across the upper and lower surfaces of the first baffle chamber, particles also begin to collide with the downstream/right side surfaces of the downstream or second baffle chamber as in figure $23 \mathrm{c}$. The gas flow into the second chamber is of reduced intensity due in part to the bleed off into the first baffle volume and, with the system geometry, the particles enter the second baffle chamber at a modified trajectory and generally a lower speed. Hence, the particle-baffle surface interactions are less intense within the second baffle chamber. The reduced erosion intensity is clear when comparing the erosion distributions at surface S10 on the right of the second baffle chamber in figure $24 \mathrm{c}$ to that at the S6 surface, on the right of the first baffle chamber, in figure $24 \mathrm{~b}$. While the maximum local erosion rate values are comparable at these two surfaces, the areas of elevated erosion are more restricted at the second chamber, to just the entrance region with little erosion over the remainder of the surface. The net erosion rate is about 1.5 times lower on S10 than on S6 on the downstream side of the first baffle.

Similar to the conditions in the first baffle chamber, the particles move along the downstream side surface of the barrel chamber, and begin to impact the top and bottom surfaces of now the second baffle chamber as seen in figure $23 \mathrm{~d}$. The highest erosion occurs near the downstream end of the top and bottom surfaces of this second chamber where the particles moving along the side surface first impact. The net erosion on the bottom S9 surface is about one-quarter of that on the S10 side surface and is about four times lower than that of the bottom of first chamber S5 (figure 24d). The weaker flow, weaker particle motion, and the specific particle-wall, particle-particle, and particle-flow interactions lead to limited erosion activity at the top and bottom surfaces of the second baffle.

The particles continue to circulate around the baffle chambers with some collisions developing at the left or upstream side surfaces of the baffle chambers as seen in figure $23 \mathrm{~d}$. However, the particle speeds have dissipated and the contact orientations are such that the erosion at these surfaces is negligible compared to that occurring on the downstream and the lower and upper surfaces of each of the baffle chambers. Some particles remain adhered at the upper right of the upper chambers and the lower right corner of the lower chambers, removing particles from potentially colliding with other surfaces.

Figure 24d summarizes the net erosion data for the four most active lower baffle surfaces (S5, S6, S9, and S10). Clearly, the greatest erosion occurs on the downstream side of the first baffle chamber, followed by the downstream side of the second baffle chamber. Erosion from the bottom (and equivalently the top) baffle surfaces is still significant, but of reduced intensity. Erosion rates decrease at the second baffle in general, due to the lower flow velocities, lower particle velocities, and the differences in the resulting particle trajectories. Adhesion effects included in the current model highlight areas of potential accumulation of particulates and the changes in the erosion rate the presence that these particulates may cause. 


\subsection{Conclusions}

The erosion rate distributions due to particle impact based erosion under dense particle concentration and large particle conditions can be studied, analyzed and better understood through the application of the unique coupled CFD-DEM-empirical based erosion rate modeling approach developed in this work. With contact models focused on capturing phenomena that dominate erosion and fouling conditions, including lower particle-object relative velocities, relative rolling, relative twisting, and adhesion effects, the method advances the state of the art, allowing for the simulations that include events such as particle-particle cohesion, particlewall adhesion, particles rolling along a surface, particles rolling over other particles, and particle resuspension into the flow.

After demonstrating good comparison to the published results, the method was applied to investigate erosion in gas-particle flow system. The results indicate that the particle size affects the erosion rate and erosion distribution, with smaller particles producing a more fluid-like particle distribution. Higher particle impact velocities generally produce higher erosion rates, but the particle size and particle interactions can significantly alter the erosion conditions. As demonstrated, in the application of the developed methods to geometries more representative of industrial processes, the techniques can be used to identify areas particularly prone to erosion and then to suggest changes to system geometries, flow, or particle conditions that produce desired erosion conditions. Further work can examine the effect of larger particle counts or different particle sizes or even mixtures of particle sizes or densities on the particle impact based erosion that develops.

The modeling method developed provides a new means to study particle/flow/flow path interactions and erosion and fouling. The basic principles used serve as the foundation for broader application of the method. Since the method utilizes the calculation of the flow induced forces from the numerical results, any viscous flow model can be implemented with the appropriate formulation of wall shear stress along the particle surface. Applications to slower speed flows and higher density fluids can readily be made, perhaps with changes to some model parameters due to the changes in the particle/flow momentum. Non-circular/nonspherical particles can be simulated with modified contact model parameters so that the changes in the flow induced forces, particle motion and particle interaction caused by the different geometry can be capture. The inclusion of chemical reactions, phase change, and interactions with other moving objects (non-particles) are other areas where the basic modeling techniques developed may be extended. The modeling approach developed within this work can serve as the basis for more comprehensive flow-particle-erosion-fouling simulation and prediction capabilities.

\section{ACKNOWLEDGEMENTS}

This work was funded by an ARDEC Technology Exploitation, Exploration, and Examination project. This work was supported in part by a grant of computer time from the DoD High Performance Computing Modernization Program at the Air Force Research Laboratory and the Naval Oceanographic Office. 


\section{REFERENCES}

[1] B.S. McLaury, Solid particle erosion in long radius elbows and straight pipes, SPE Annual Technical Conference and Exhibition (2014) 19-37.

[2] J.H. Kim H.G. Joo, K.Y. Lee., Solid particles under airjet colliding with WC-Ni coated specimens. Journal of Materials Processing Technology 224 (2015) 240-245.

[3] L..J.W Graham, D.R. Lester, and J. Wu, Quantification of erosion distributions in complex geometries. Wear 268 (2010) 1066-1071.

[4]Solnordal, C.B. et al., Determintation of erosion rate characteristics for particles with size distribution in the low Stokes number range. Wear 305 (2013) 205-215.

[5]Wong, et. al. Experimental and computational modeling of solid particle erosion in a pipe annular cavity. Wear 303 (2013) 109-129.

[6] E. Simek et. al. Numerical simulation of grate firing systems using a coupled CFD/discrete element method. Powder Technology 193 (2009) 266-273.

[7] Y. Tsuji, T. Tanaka, T. Ishida, Lagrangian numerical simulation of plug flow of cohesionless particles in a horizontal pipe, Powder Technology 71(3) (1992) 239-250.

[8] J. Chen et. al., Erosion prediction of liquid -particle two phase flow in pipline elbows via CFD-DEM coupling method. Powder Technology 275(2015) 182-187.

[9]M. Jafari et al., The effects of wall roughness on erosion rate in gas-solid turbulent annular pipe flow, Powder Technology 271(2015) 248-254.

[10] L.A. Florio, Direct modeling of coupled compressible flow and macroscopic particle spread and particle ejection Mecannica 50(9) (2015) 2257-2291.

[11] L.A. Florio, Dynamic coupling of fluid and structural mechanics for simulation of particle motion and interaction in high speed compressible gas particle laden flow Journal of Fluids and Structures 54 (2015)171-201.

[12] L.A. Florio, Computation of fluid-particle interactions with high-speed compressible flows and multiple particles with deformation, plasticity, and collision, Powder Technology 268 (2014) 19-37.

[13]L.A. Florio Effect of gas equation of state on CFD predictions for ignition characteristics of hydrogen escaping from a tank, International Journal of Hydrogen Energy 39(32) (2014) 18451-18471.

[14] F.M. White, Viscous Fluid Flow, McGraw HIII, New York, 1991.

[15] T.-H. Shih, et. al., A new $\kappa-\varepsilon$ eddy-viscosity model for high Reynolds number turbulent flows-model development and validation, Computers and Fluids 24 (1995) 227-238.

[16]Fluent Theory Guide v16.0, 2015.

[17]H-G Matuttis, J. Chen. Understanding the Discrete Element Method, Wiley, Singapore, 2014.

[18]]J.S. Marshall, S. Li, Adhesive Particle Flow, Cambridge University Press, New York, 2014.

[19]..H. Shames, Engineering Mechanics: Dynamics, Prentice Hall, New York, 1996.

[20]B.P. Ickes, A new method for performing digital control system attitude computaions using quaternions, AIAA Journal 8 (1970) 13-17.

[21]R. Okita, Y. Zhang, B.S. McLaury, S. A. Shirazi, Experimental and computational investigations to evaluate the effects of fluid viscosity and particle size on erosion damage, Journal of Fluids Engineering 134(2012) 061301-1-13.

[22] S.V. Patankar, Numerical Heat Transfer and Fluid Flow, Hemisphere Publishing, New York, 1980.

[23]A. Gnanavelu et al., A numerical investigation of a geometry independent integrated method to predict erosion rates in slurry erosion, Wear 271 (2011) 712-719. 


\section{UNCLASSIFIED}

\section{APPENDIX}

The transformation matrix from the local body coordinate system of particle $\mathrm{x}$ to the global coordinate system $\mathrm{G}, \mathrm{R}^{\mathrm{xG}}$, is defined below using the components of the quaternion associated with the rotational motion of object $\mathrm{x}, \bar{E}^{\mathrm{x}}$. Further details on the derivation can be found in [15] and [18].

$$
\begin{aligned}
R^{x G}[0][0]= & E^{x}[0] E^{x}[0]+E^{x}[1] E^{x}[1]-E^{x}[2] E^{x}[2]-E^{x}[3] E^{x}[3] \\
& R^{x G}[0][1]=2\left(E^{x}[1] E^{x}[2]-E^{x}[0] E^{x}[3]\right) \\
& R^{x G}[0][2]=2\left(E^{x}[1] E^{x}[3]+E^{x}[0] E^{x}[2]\right) \\
& R^{x G}[1][0]=2\left(E^{x}[1] E^{x}[2]+E^{x}[0] E^{x}[3]\right) \\
R^{x G}[1][1]= & E^{x}[0] E^{x}[0]-E^{x}[3] E^{x}[3]-E^{x}[1] E^{x}[1]+E^{x}[2] E^{x}[2] \\
& R^{x G}[1][2]=2\left(E^{x}[3] E^{x}[2]-E^{x}[0] E^{x}[1]\right) \\
& R^{x G}[2][0]=2\left(E^{x}[3] E^{x}[1]-E^{x}[0] E^{x}[2]\right) \\
& R^{x G}[2][1]=2\left(E^{x}[0] E^{x}[1]+E^{x}[2] E^{x}[3]\right) \\
R^{x G}[2][2]= & E^{x}[0] E^{x}[0]+E^{x}[3] E^{x}[3]-E^{x}[1] E^{x}[1]-E^{x}[2] E^{x}[2]
\end{aligned}
$$

Using the local body rotational velocity, $\bar{\Omega}_{x \times}^{x}$.

$$
\begin{aligned}
& \dot{E}^{x}[0]=1 / 2\left(E^{x}[1] \Omega_{x}^{x}[0]-E^{x}[2] \Omega_{x}^{x}[1]-E^{x}[3] \Omega_{x}^{x}[2]\right) \\
& \dot{E}^{x}[1]=1 / 2\left(E^{x}[0] \Omega_{x}^{x}[0]-E^{x}[3] \Omega_{x}^{x}[1]-E^{x}[2] \Omega_{x}^{x}[2]\right) \\
& \dot{E}^{x}[2]=1 / 2\left(E^{x}[3] \Omega_{x}^{x}[0]+E^{x}[0] \Omega_{x}^{x}[1]-E^{x}[1] \Omega_{x}^{x}[2]\right) \\
& \dot{E}^{x}[2]=1 / 2\left(E^{x}[2] \Omega_{x}^{x}[0]+E^{x}[1] \Omega_{x}^{x}[1]+E^{x}[0] \Omega_{x}^{x}[2]\right)
\end{aligned}
$$

Then, the quaternion can be updated for the new time step as:

$$
\bar{E}_{t+\Delta t}^{x}=\overline{\dot{E}}^{x} \Delta t+\bar{E}_{t}^{x}
$$

\section{Vitae}

L. A. Florio is a mechanical engineer in the Weapon Technology Branch of the Small Caliber Armaments Division at ARDEC in Picatinny Arsenal, NJ. She holds a Ph.D. in mechanical engineering in the area of computational heat transfer and fluid flow. She has been developing capabilities to simulate and analyze a wide range of fluid/multiphase flow and heat transfer phenomenon in small arms systems. 


\section{UNCLASSIFIED}

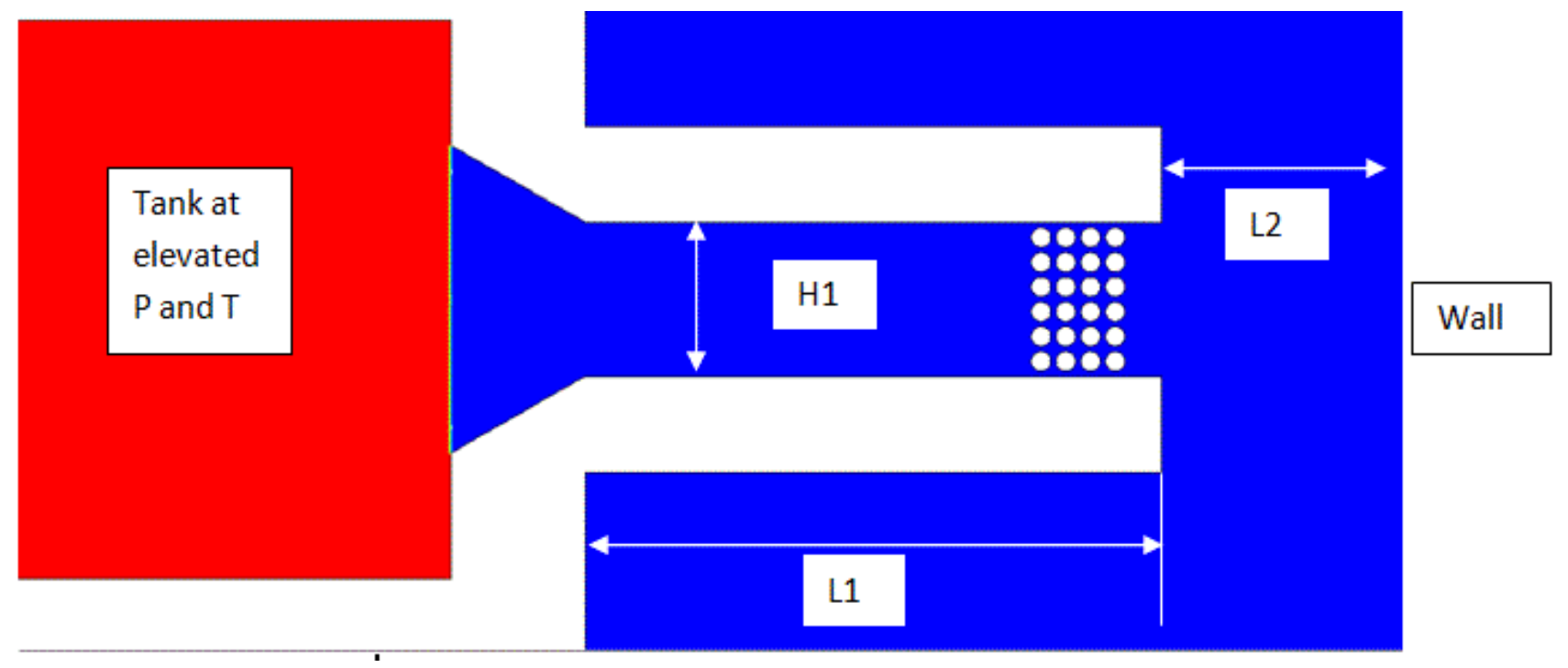

a)System investigated
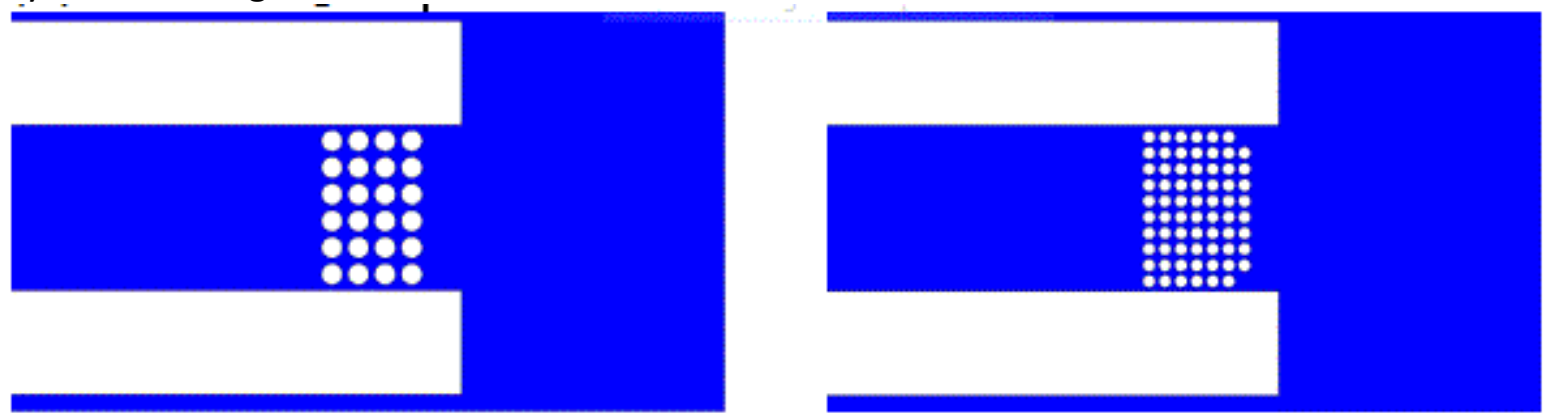

b) 24 particles, $1.01 \mathrm{~mm}$ diameter system c) 68 particles, $0.60 \mathrm{~mm}$ diarneter system

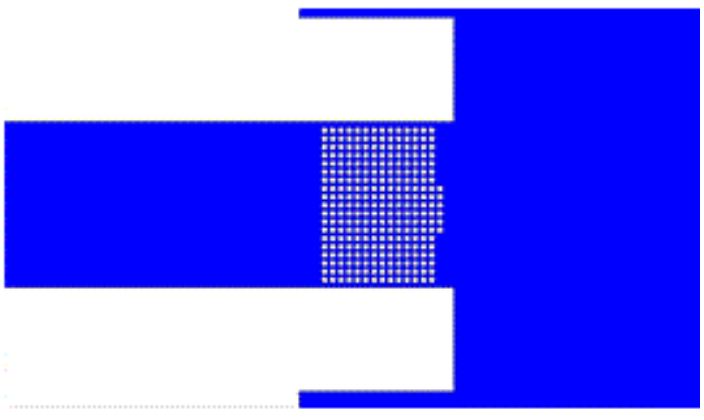

d) 272 particles, $300 \mu \mathrm{m}$ diameter system

Figure 1. Sand particles exiting a flow path and impacting fixed perpendicular Aluminum plate 


\section{UNCLASSIFIED}

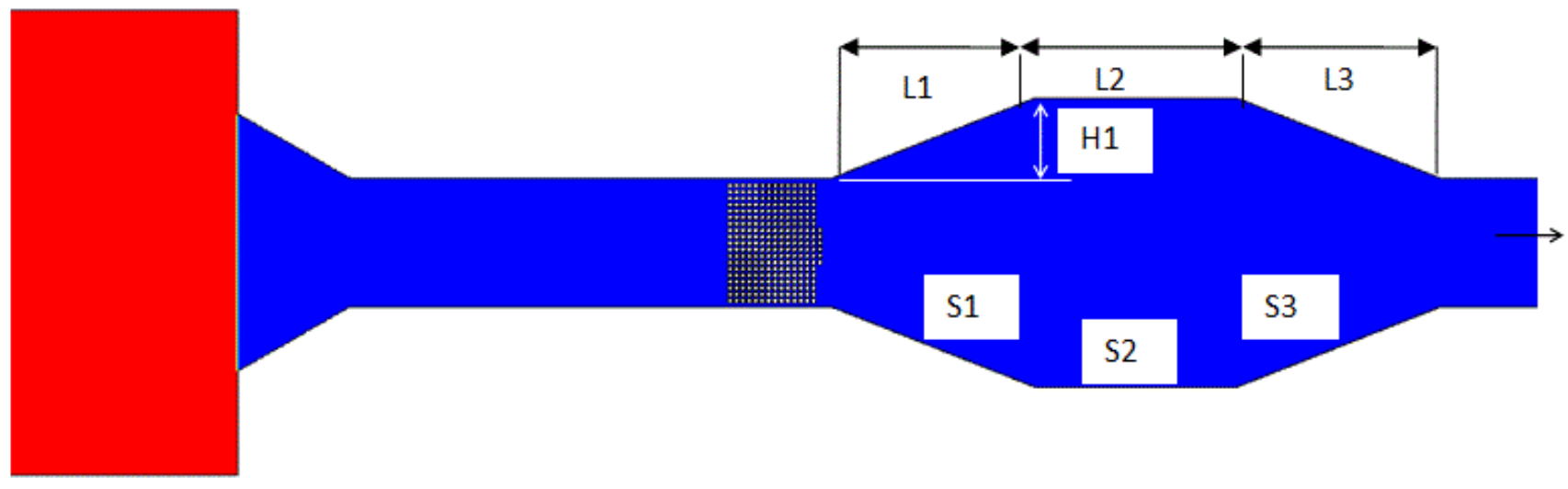

a) Diverging-converging configuration

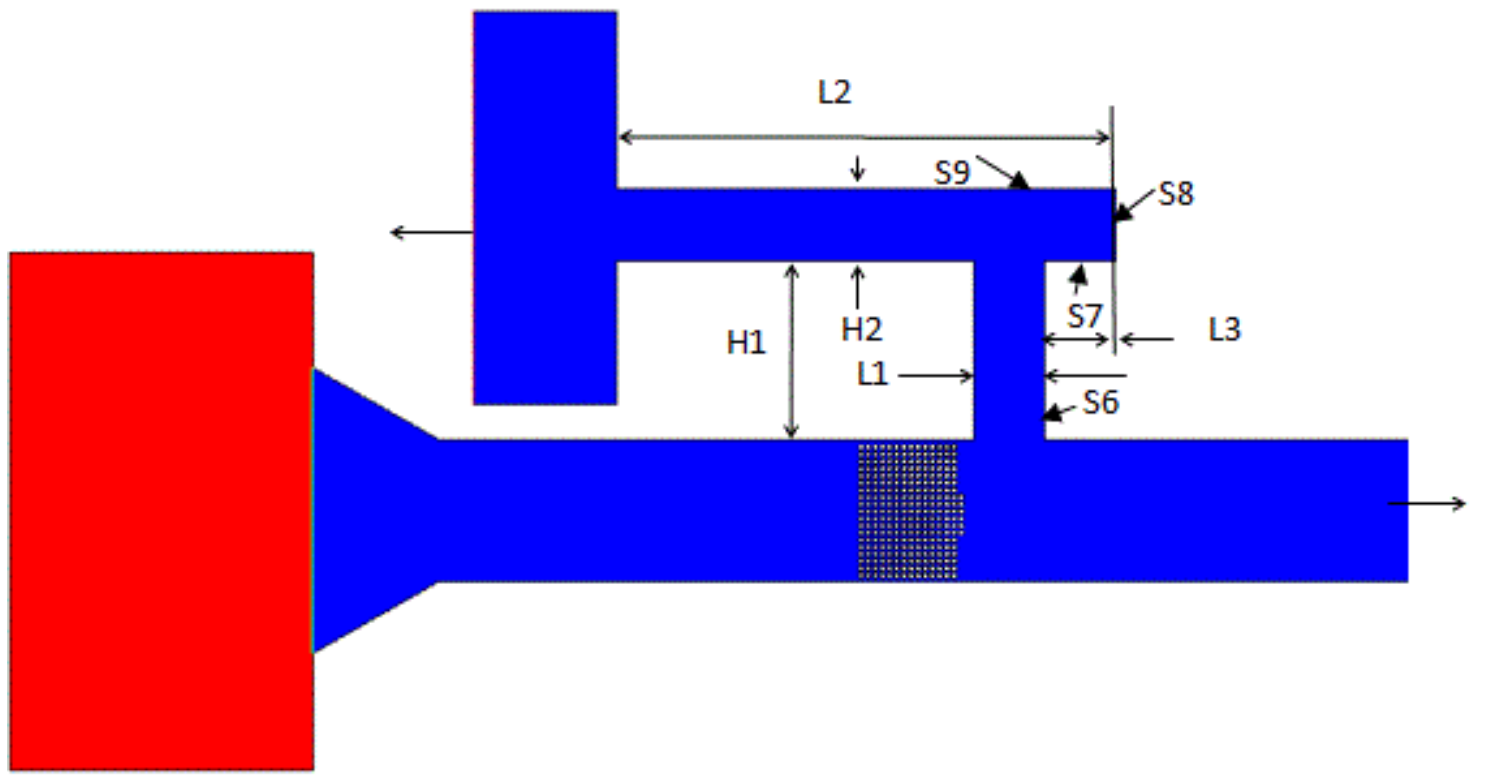

b) By-pass configuration

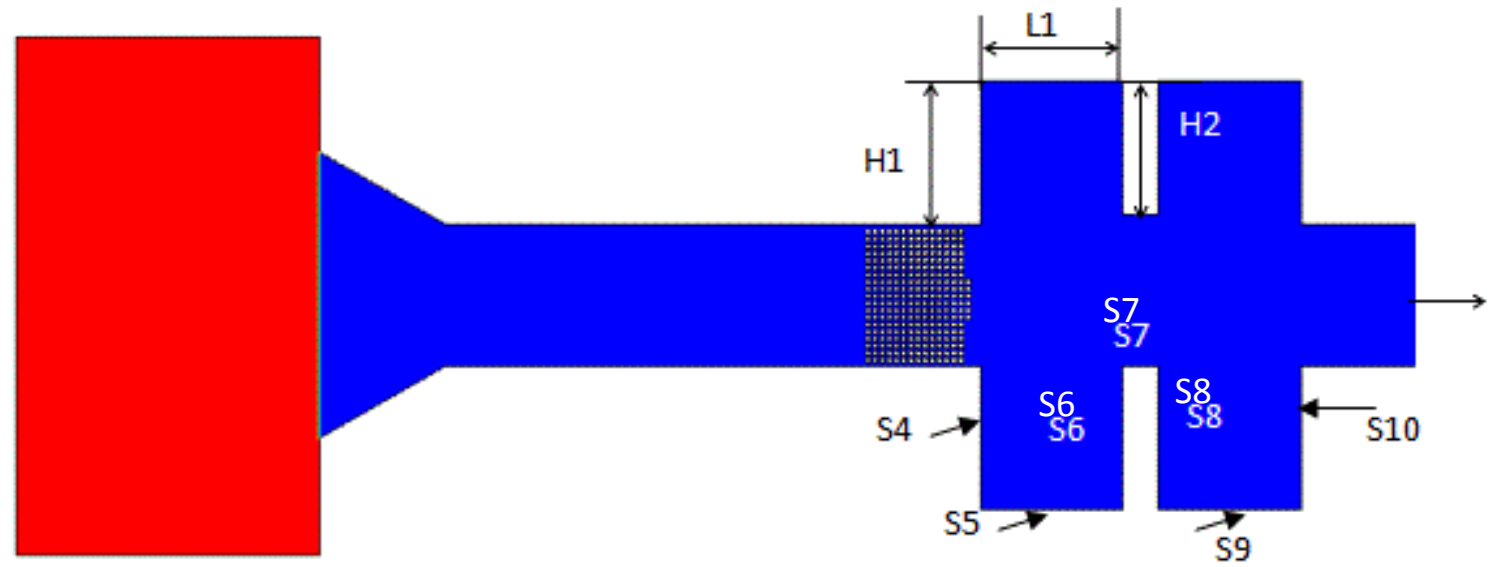

c) Baffle configuration

Figure 2. Sand particles impacting Aluminum surfaces for three different system configurations, $272300 \mu m$ diameter particles 


\section{UNCLASSIFIED}

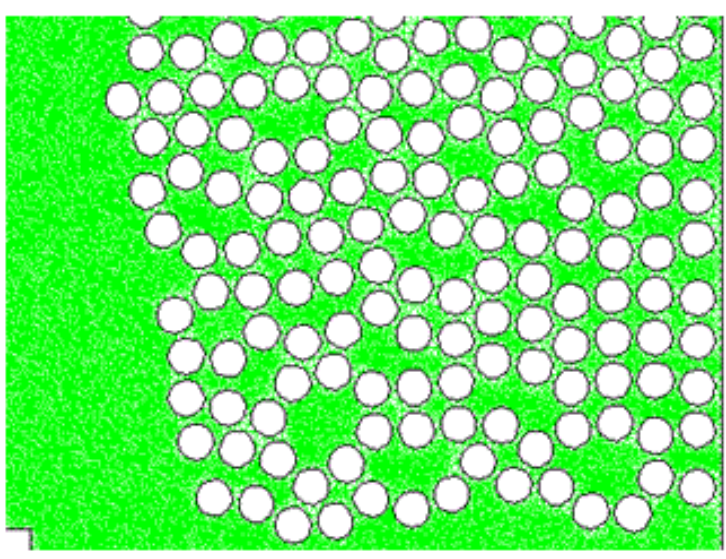

a)Overall view of particle surface discretization

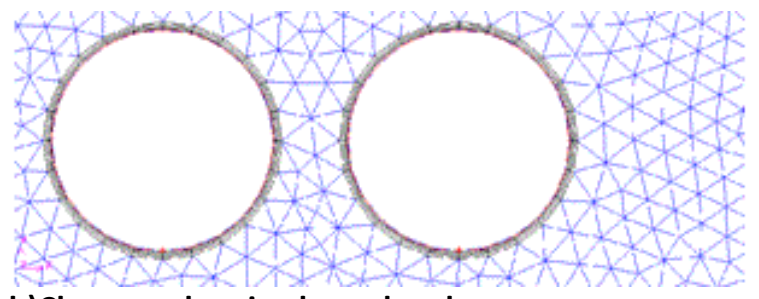

b)Close-up showing boundary layer

Figure 3. Particle discretization within the computational fluid dynamics domain


Figure 4. Two colliding particles, vectors defined

b)Start of collision

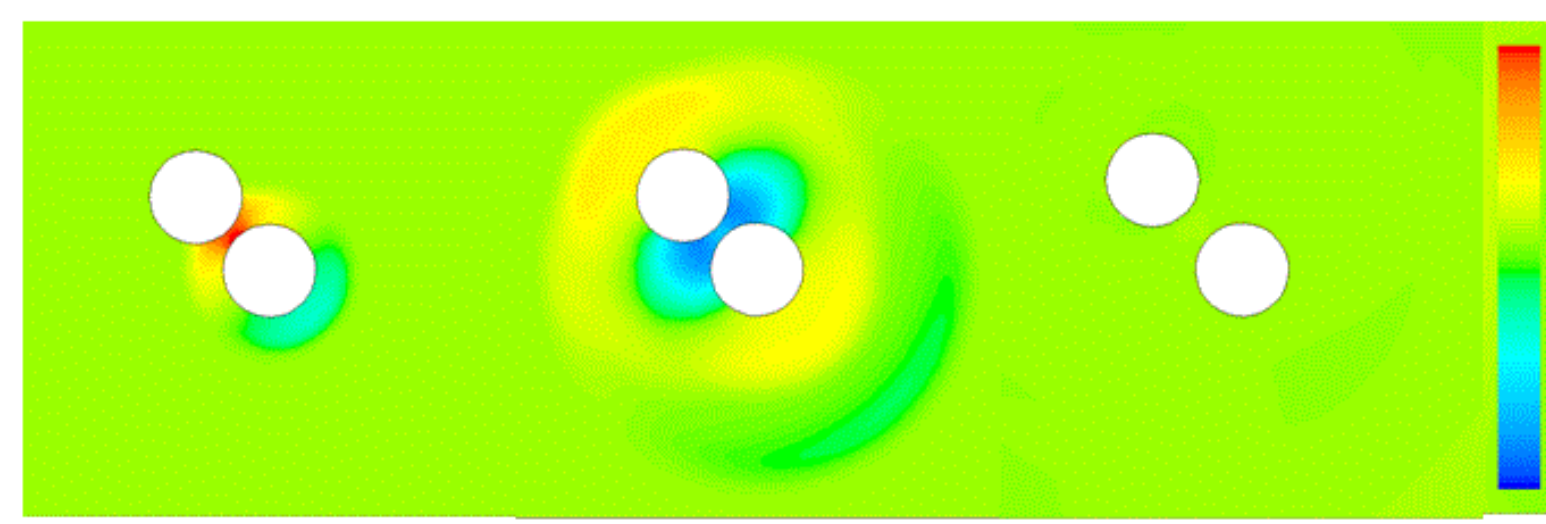

$\mathrm{Pa}$

a)Pressure contours for elastic normal contact model(Pressure in $\mathrm{Pa}$ ) 


\section{UNCLASSIFIED}

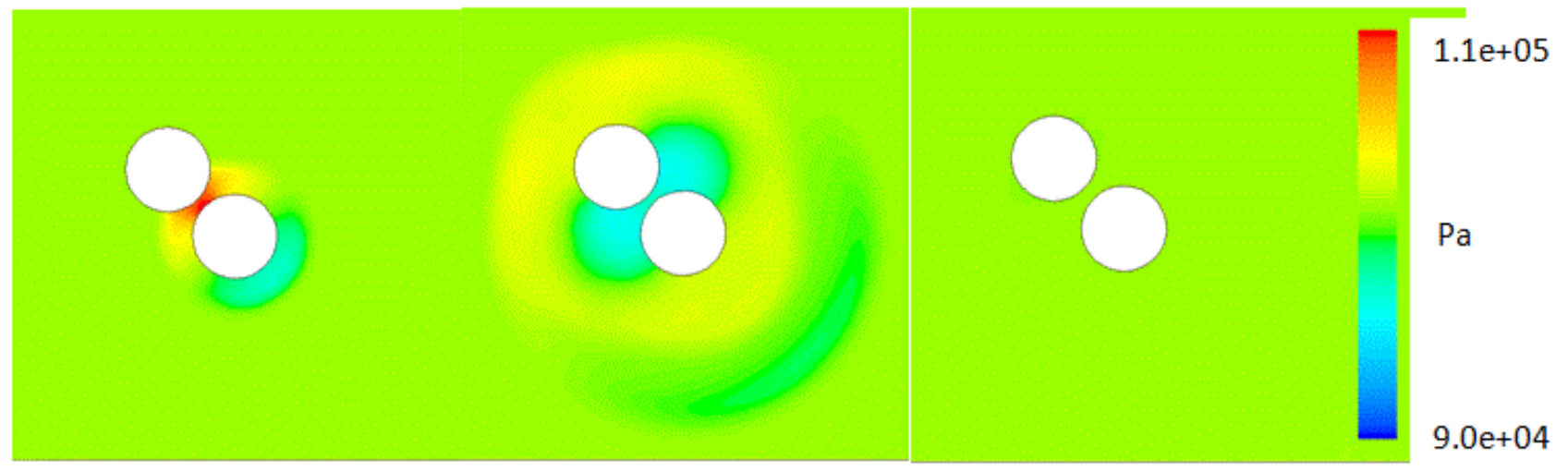

b) Pressure contours for normal contact model with damping(Pressure in Pa)

Figure 5. Pressure contours demonstrating effect of damping in contact model

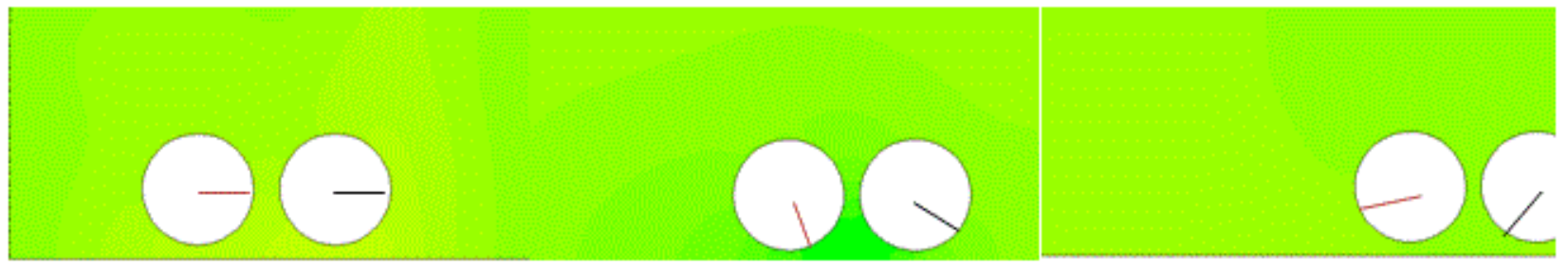

Figure 6. Rotation of particles as they roll along a fixed wall
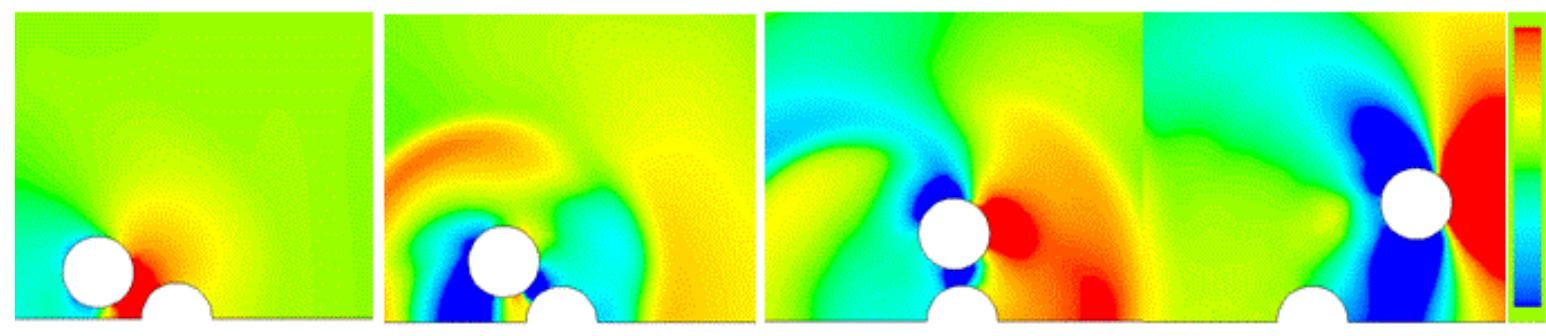

$\mathrm{Pa}$

$9.0 \mathrm{e}+04$

a)Pressure contours with no cohesion force, including rolling effect (Pressure in $\mathrm{Pa}$ )
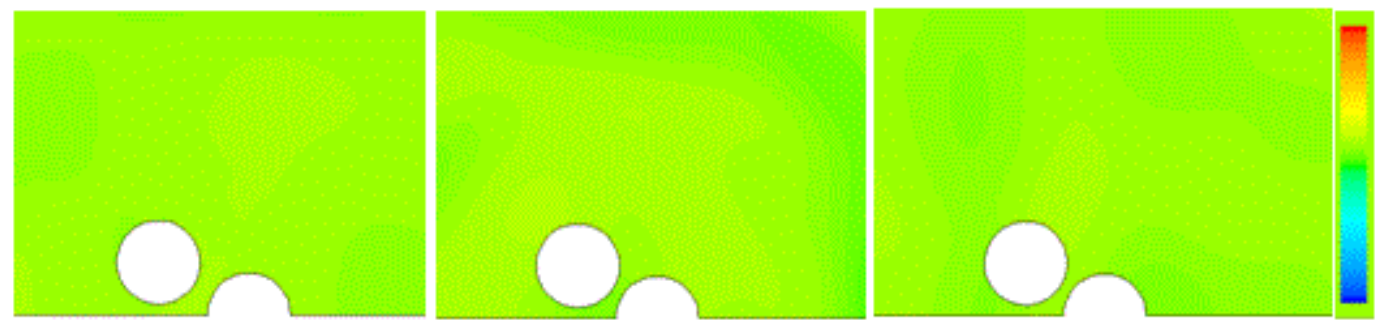

$1.1 \mathrm{e}+05$

$\mathrm{Pa}$

$9.0 \mathrm{e}+04$

b)Pressure contours for strong cohesion force (Pressure in $\mathrm{Pa}$ )

Figure 7. Cohesion effects for single particle interaction with wall and wall roughness element 


\section{UNCLASSIFIED}

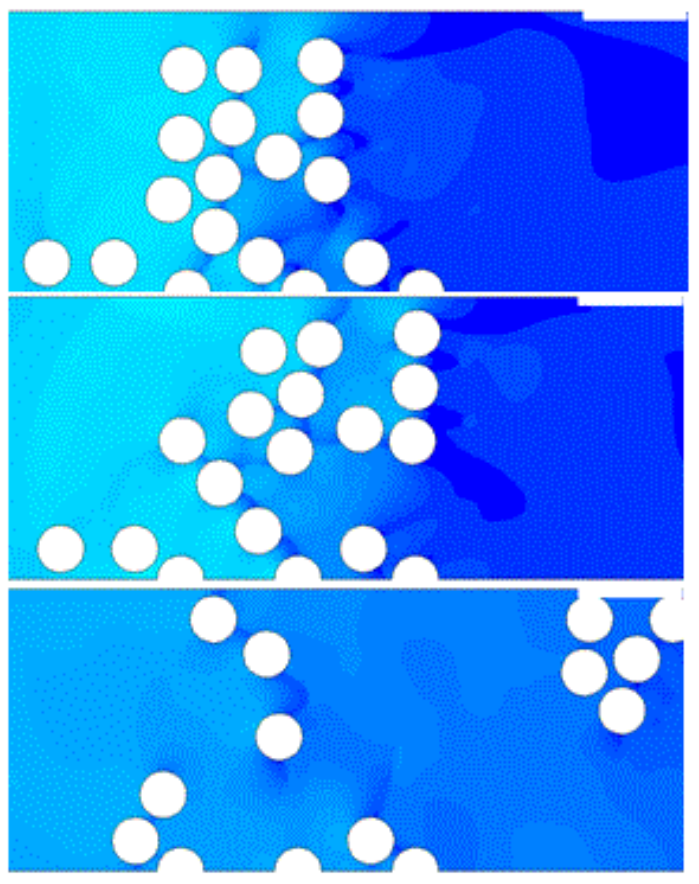

a)Lower cohesion strength

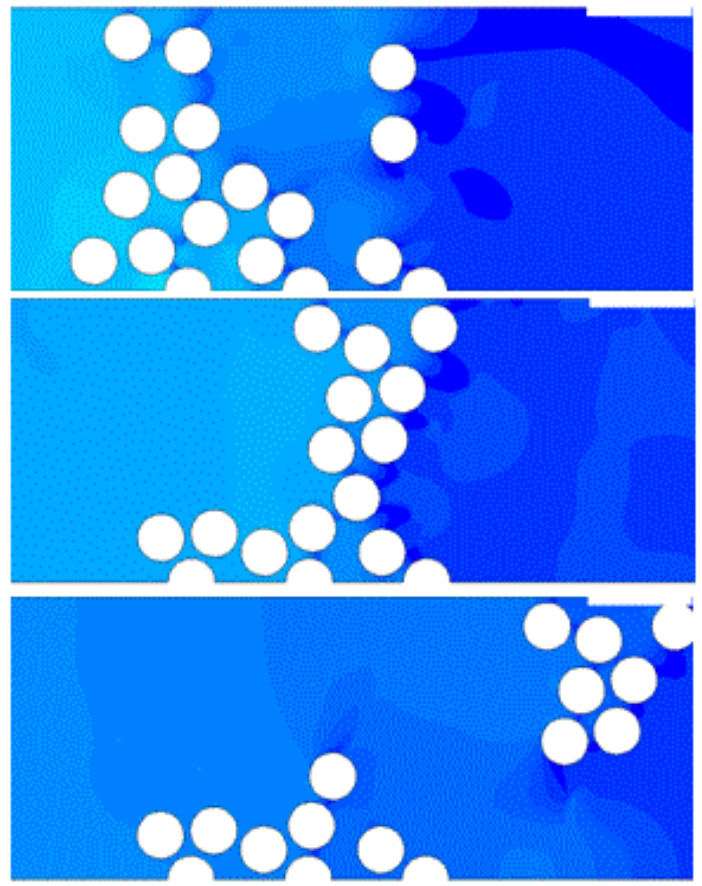

b) Higher cohesion strength

Figure 8. Cohesion effects for multi-particle interaction with wall with roughness elements: Pressure contour sequence with pressures in $\mathrm{Pa}$.

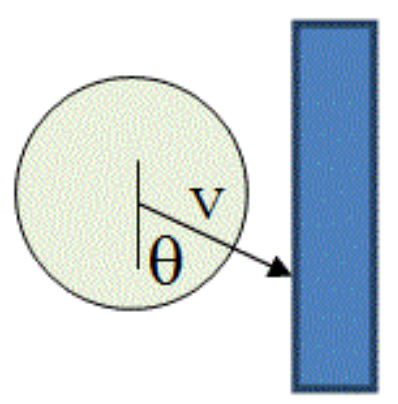

Figure 9. Velocity and impact angle definitions 


\section{UNCLASSIFIED}

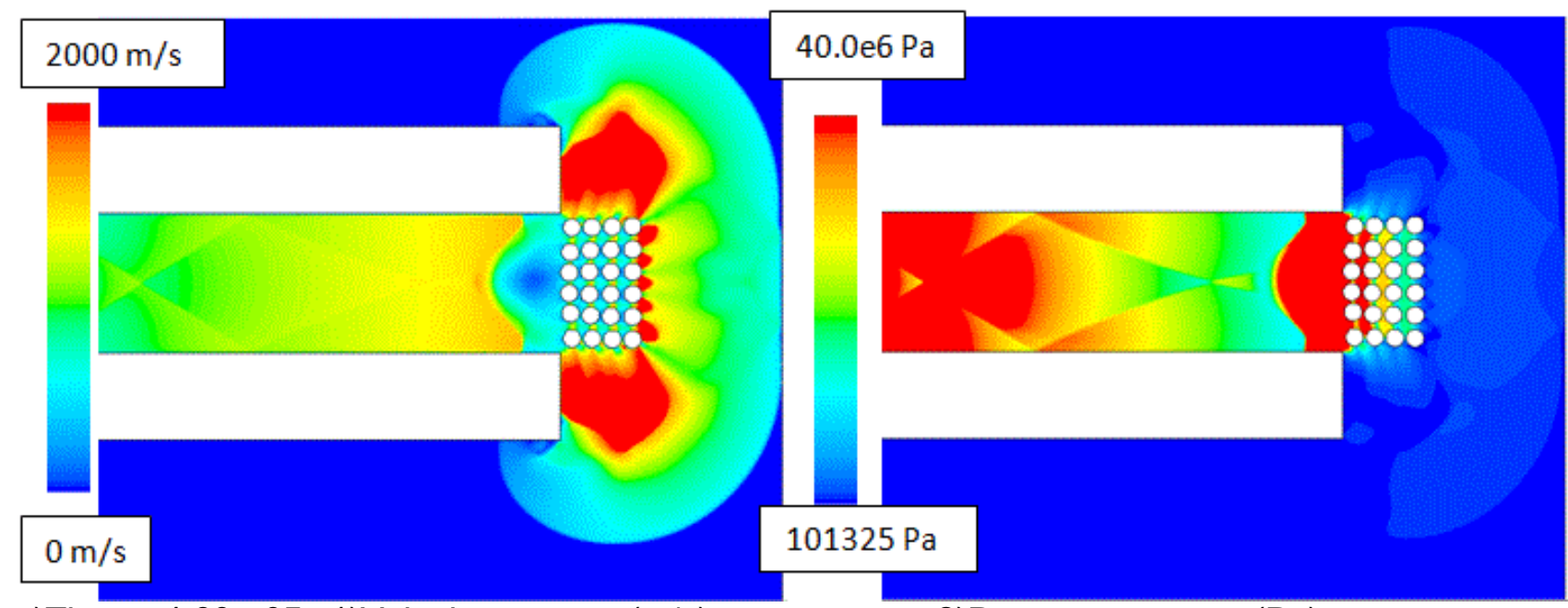

a) Time $=4.60 \mathrm{e}-05 \mathrm{~s} 1)$ Velocity contours $(\mathrm{m} / \mathrm{s})$

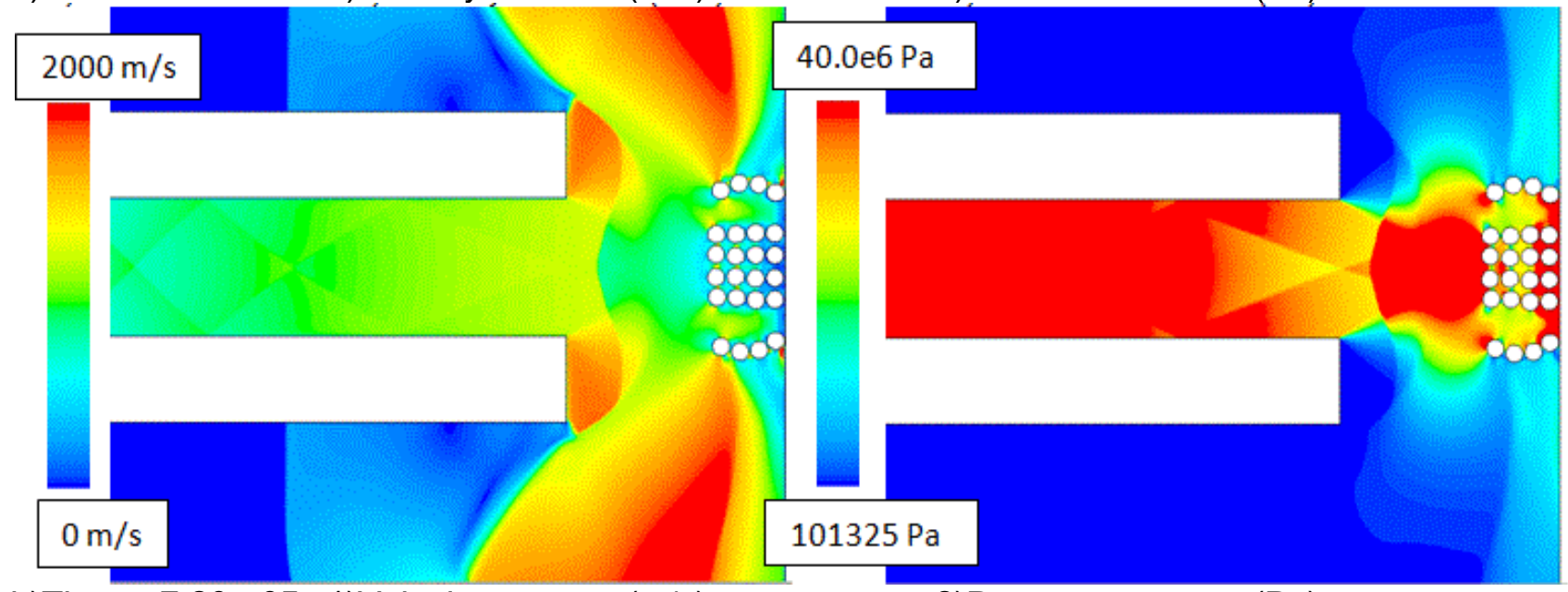

b) Time $=7.20 \mathrm{e}-05 \mathrm{~s} 1)$ Velocity contours $(\mathrm{m} / \mathrm{s})$

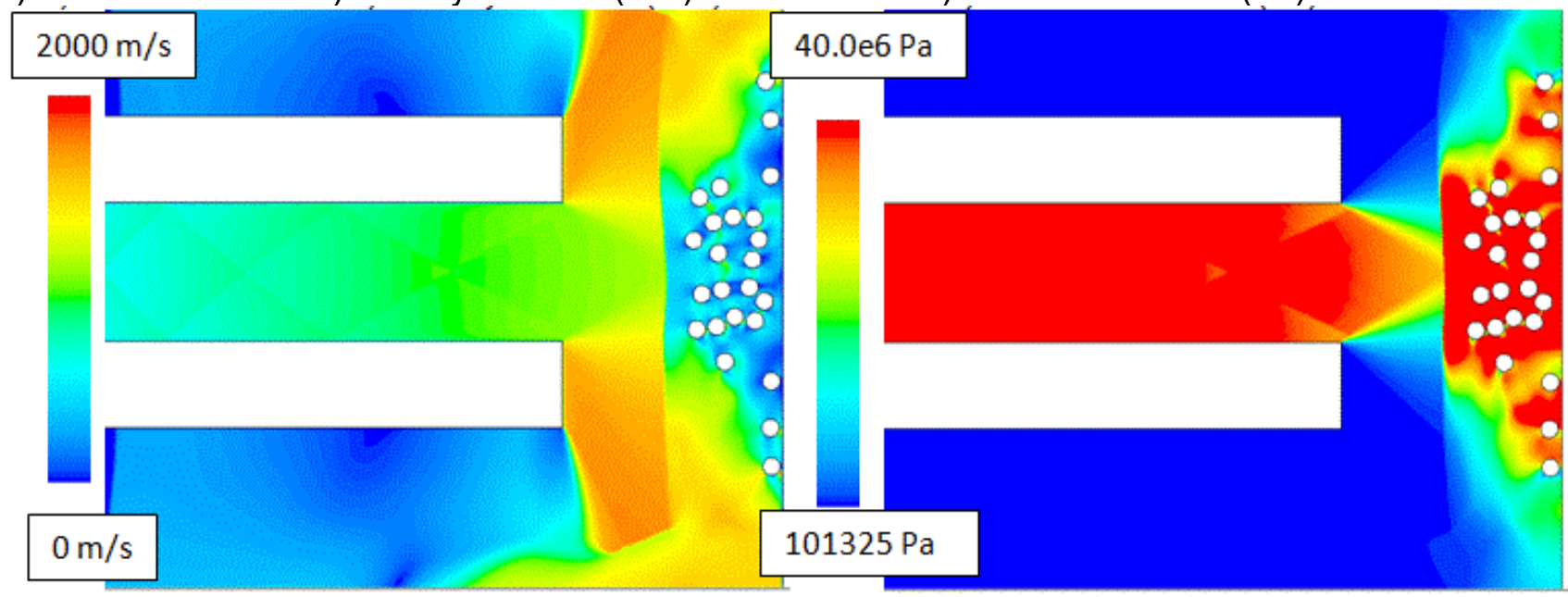

c) Time $=9.00 \mathrm{e}-05 \mathrm{~s} 1)$ Velocity contours $(\mathrm{m} / \mathrm{s})$

2)Pressure contours $(\mathrm{Pa})$

Figure 10. Velocity and pressure contours for case of $241.01 \mathrm{~mm}$ diameter particles(continued) 


\section{UNCLASSIFIED}

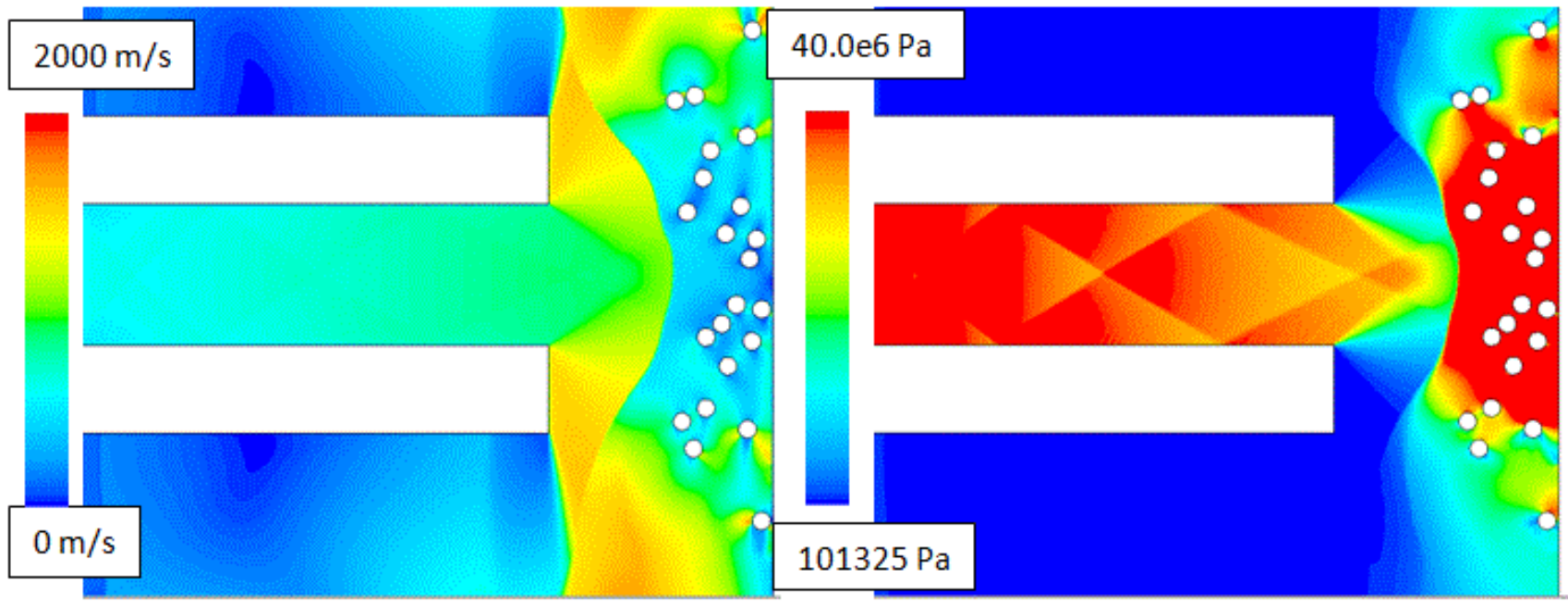

d) Time $=1.10 \mathrm{e}-04 \mathrm{~s} 1)$ Velocity contours $(\mathrm{m} / \mathrm{s})$

2)Pressure contours $(\mathrm{Pa})$

Figure 10. Velocity and pressure contours for case of $241.01 \mathrm{~mm}$ diameter particles

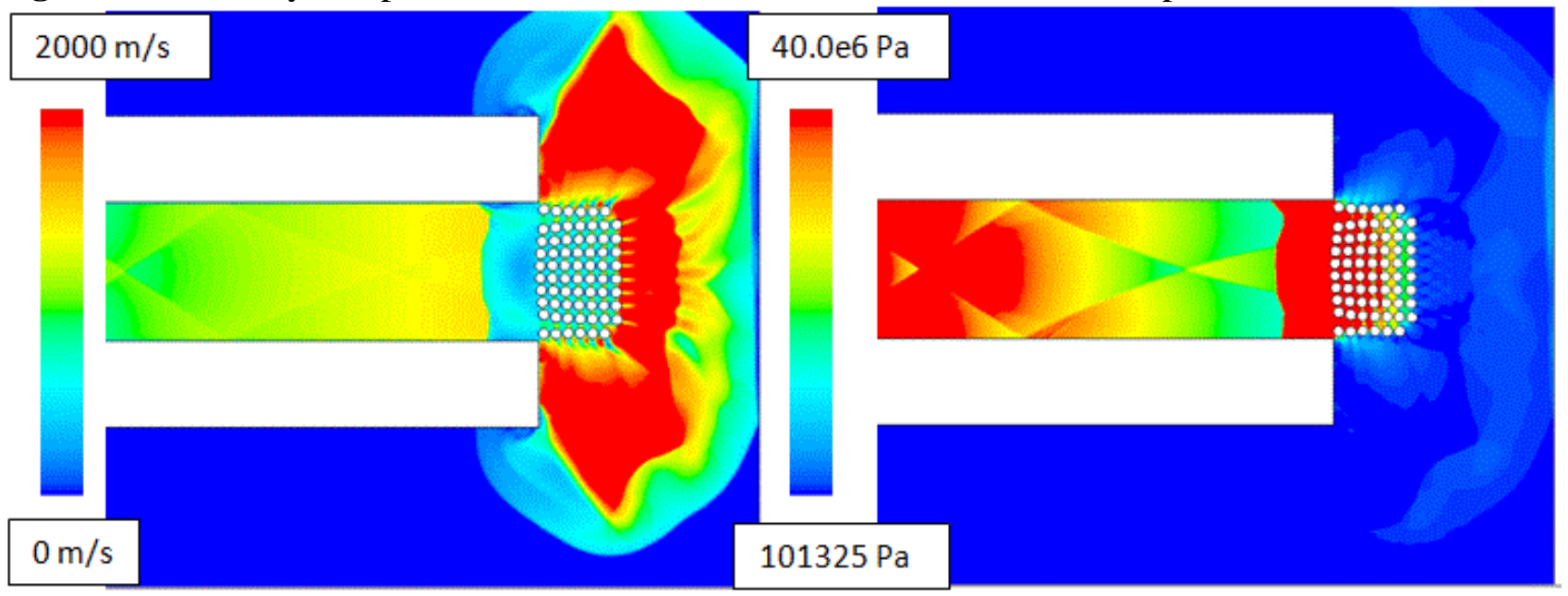

a) Time $=4.40 \mathrm{e}-05 \mathrm{~s} 1)$ Velocity contours $(\mathrm{m} / \mathrm{s})$

2)Pressure contours $(\mathrm{Pa})$

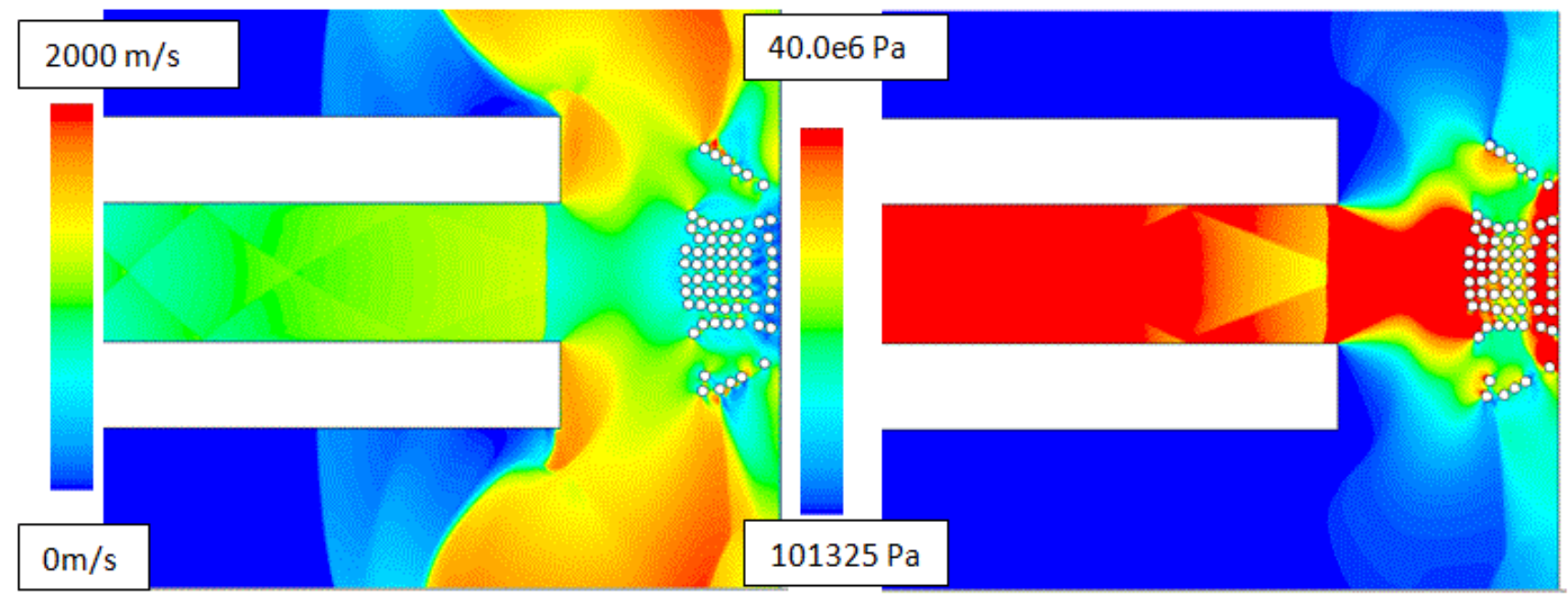

b) Time $=6.60 \mathrm{e}-05 \mathrm{~s} 1)$ Velocity contours $(\mathrm{m} / \mathrm{s})$

2)Pressure contours $(\mathrm{Pa})$

Figure 11. Velocity and pressure contours for case of $680.60 \mathrm{~mm}$ diameter particles(continued) 


\section{UNCLASSIFIED}

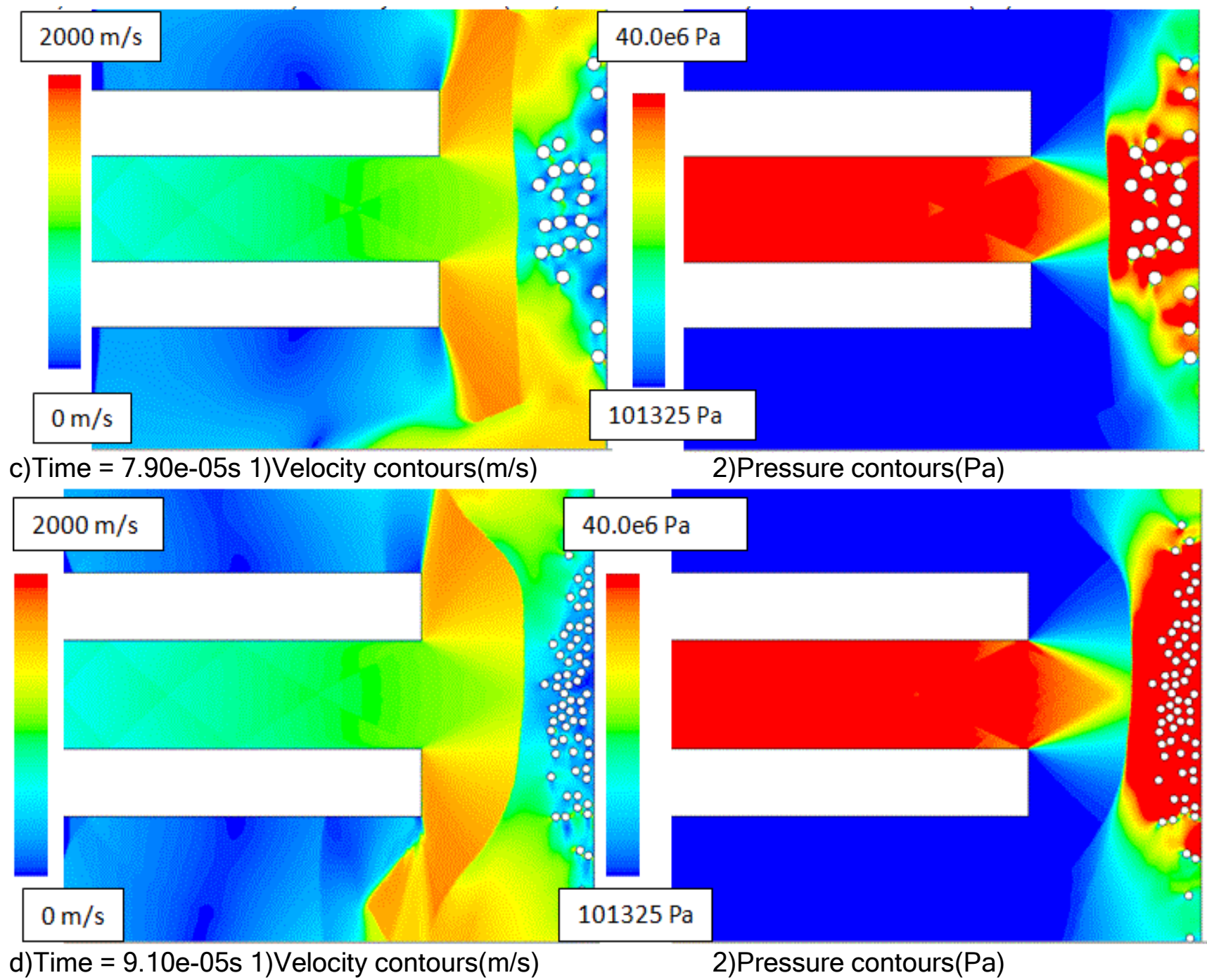

Figure 11. Velocity and pressure contours for case of $680.60 \mathrm{~mm}$ diameter particles

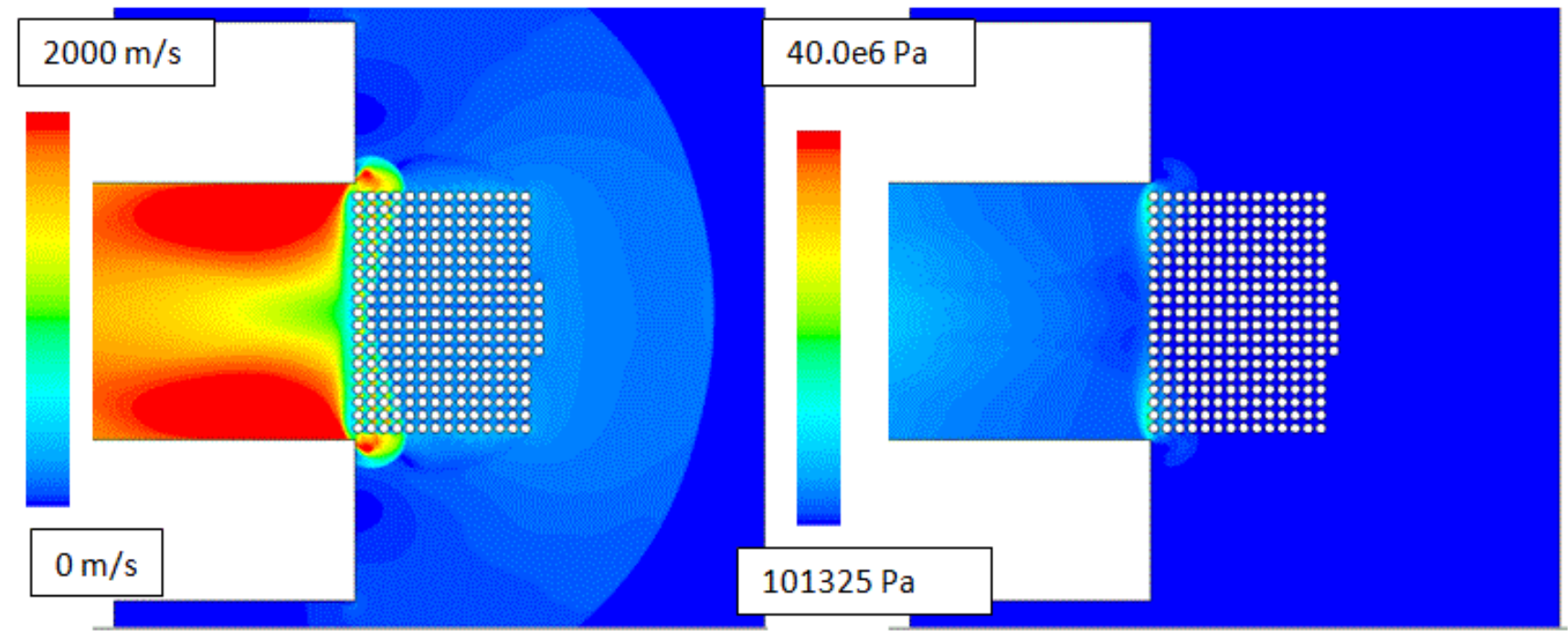

a) Time $=3.25 \mathrm{e}-05 \mathrm{~s} 1)$ Velocity contours $(\mathrm{m} / \mathrm{s})$

2)Pressure contours $(\mathrm{Pa})$

Figure 12. Velocity and pressure contours for case of $2720.30 \mathrm{~mm}$ diameter particles(continued) 


\section{UNCLASSIFIED}

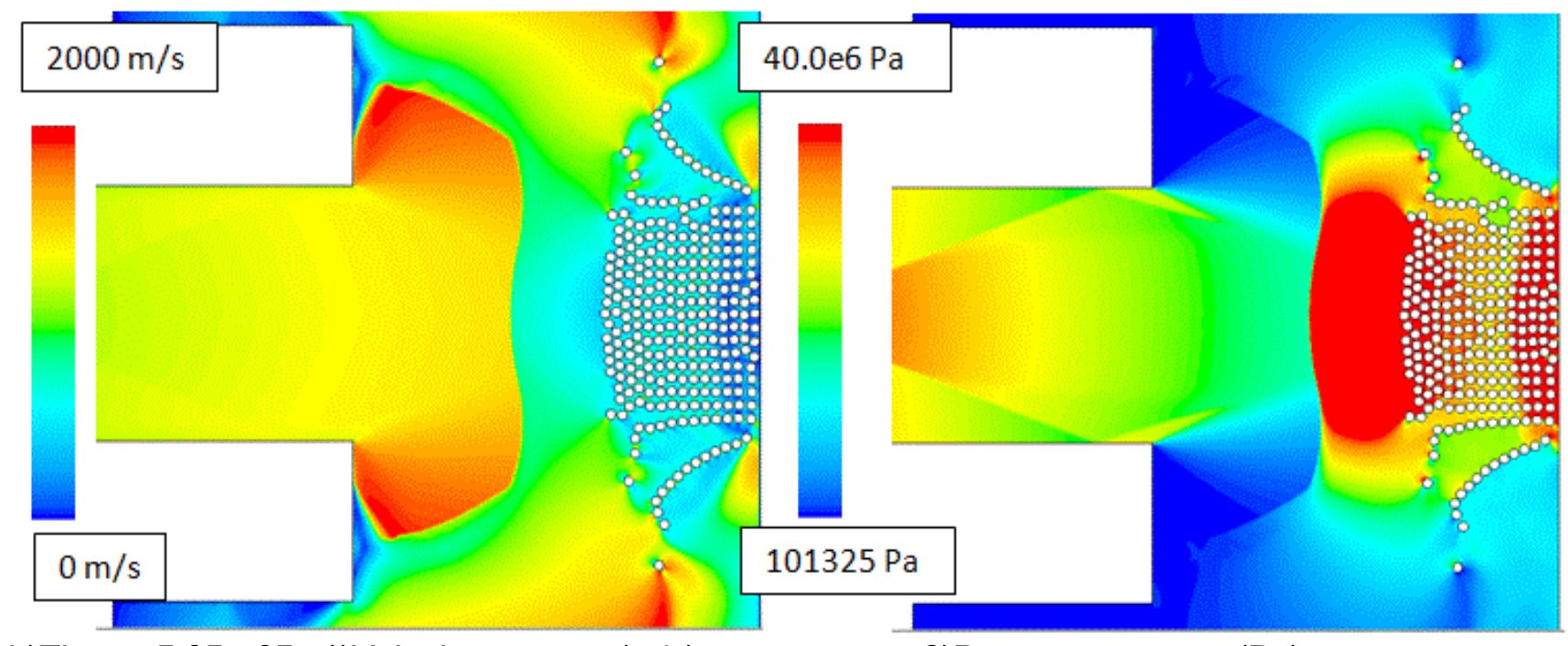

b) Time $=5.95 \mathrm{e}-05 \mathrm{~s} 1)$ Velocity contours $(\mathrm{m} / \mathrm{s})$

2)Pressure contours $(\mathrm{Pa})$

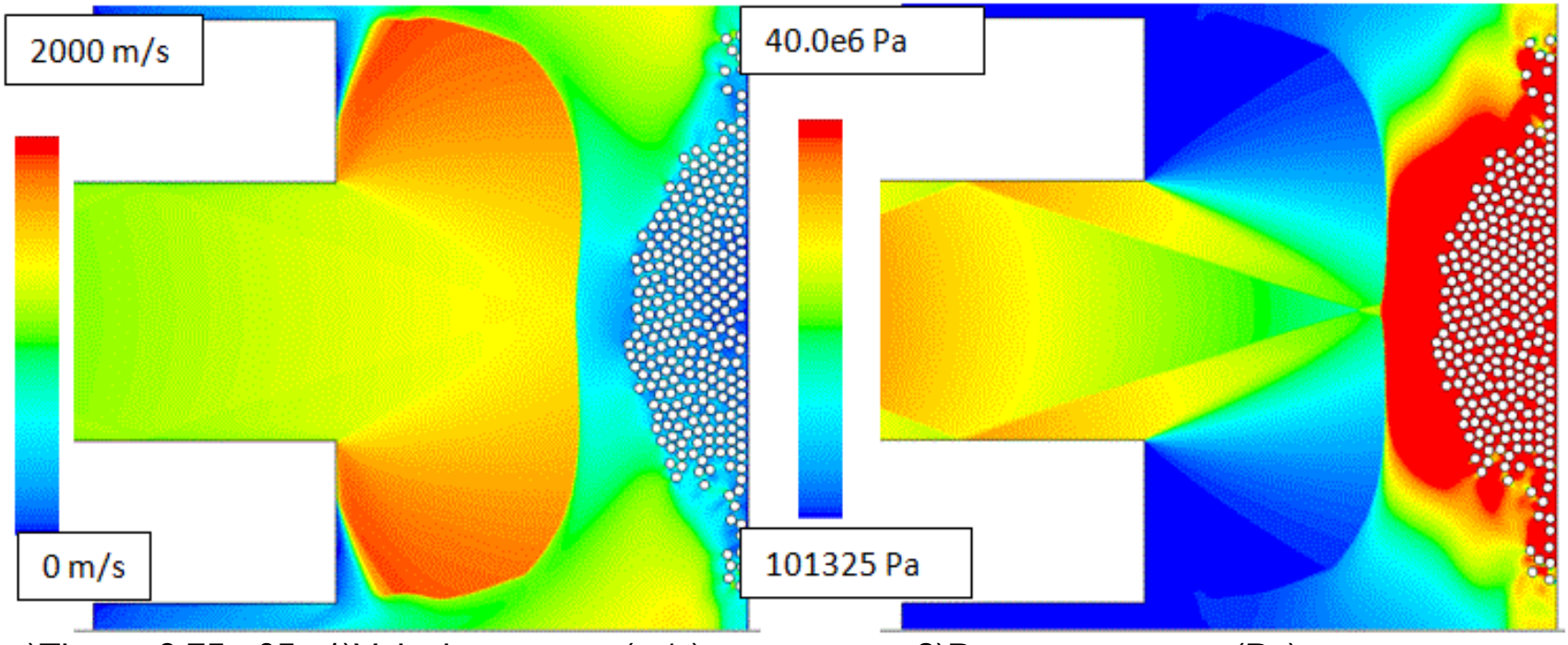

c)Time $=6.75 \mathrm{e}-05 \mathrm{~s} 1)$ Velocity contours $(\mathrm{m} / \mathrm{s})$

2)Pressure contours(Pa)



Figure 12. Velocity and pressure contours for case of $272300 \mu \mathrm{m}$ diameter particles 


\section{UNCLASSIFIED}

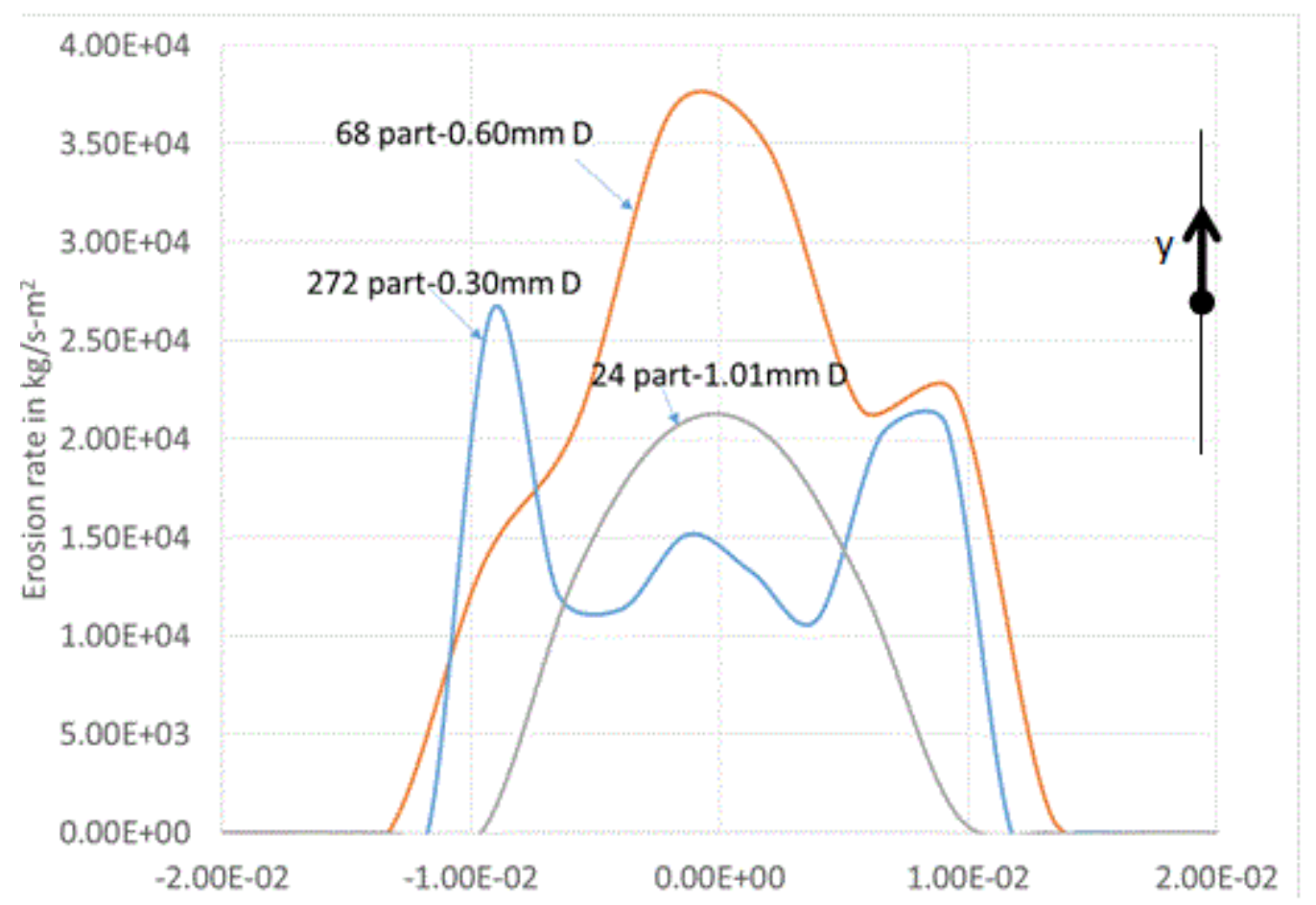

Figure 13. Erosion distribution at $1.0 \mathrm{e}-04$ seconds for indicated particle size cases 


\section{UNCLASSIFIED}

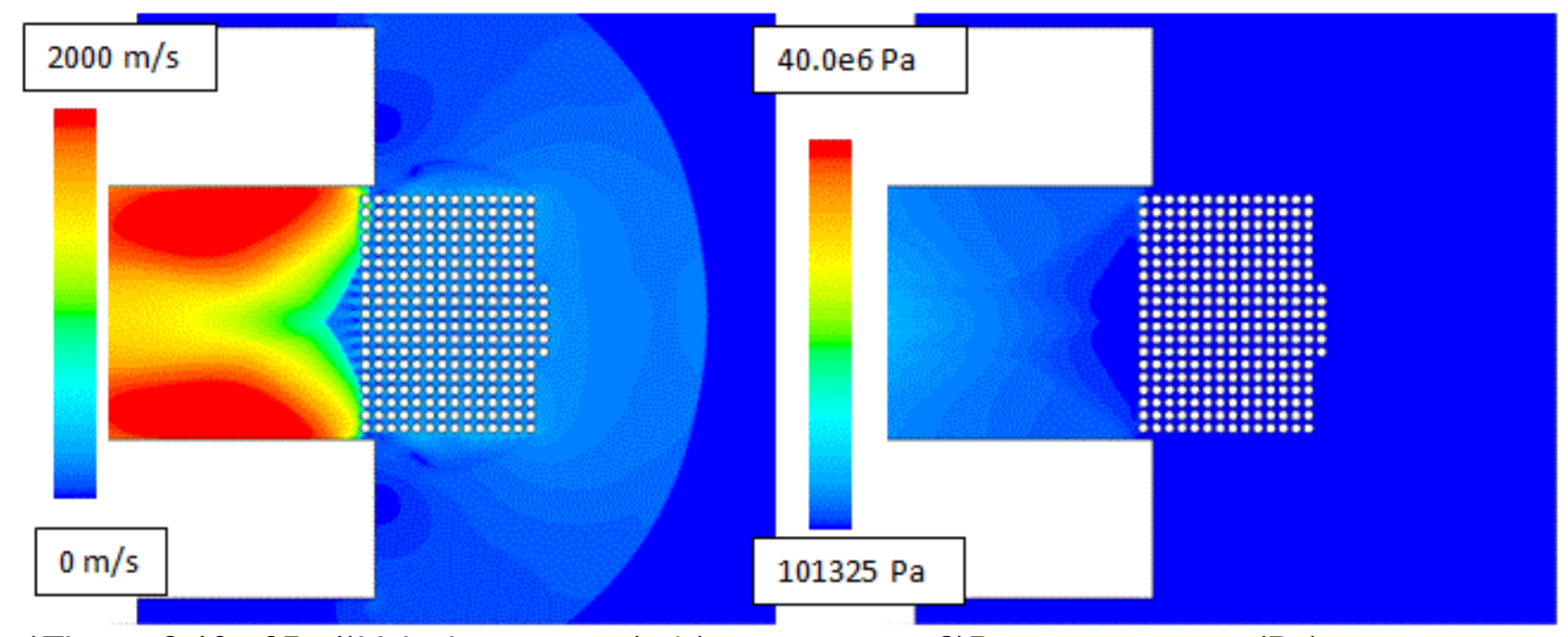

a) Time $=3.10 \mathrm{e}-05 \mathrm{~s} 1)$ Velocity contours $(\mathrm{m} / \mathrm{s})$

2)Pressure contours(Pa)



b) Time $=3.70 \mathrm{e}-05 \mathrm{~s} 1)$ Velocity contours $(\mathrm{m} / \mathrm{s})$

2)Pressure contours $(\mathrm{Pa})$

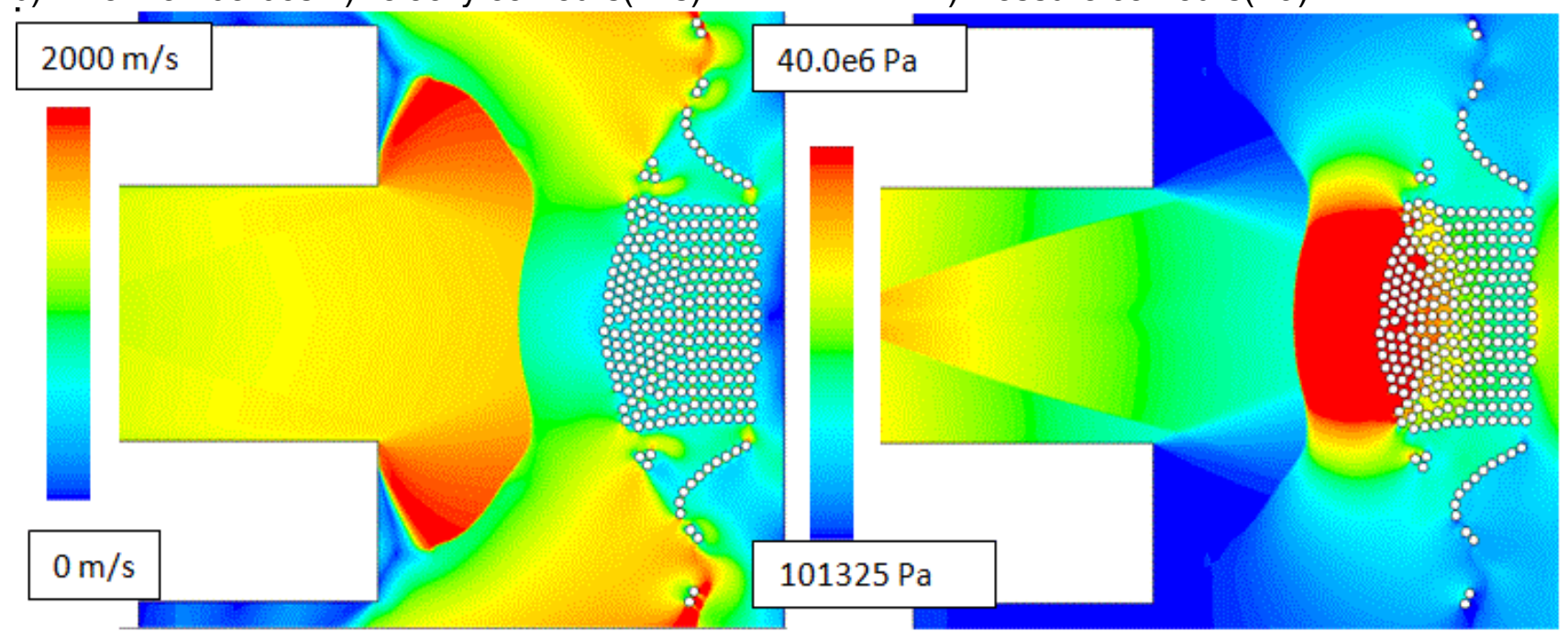

c) Time $=5.50 \mathrm{e}-05 \mathrm{~s} 1)$ Velocity contours $(\mathrm{m} / \mathrm{s})$

2)Pressure contours(Pa)

Figure 14. Velocity and pressure contours for case of $272300 \mu \mathrm{m}$ diameter particles, initial velocity of $250 \mathrm{~m} / \mathrm{s}$ - average impact velocity of $430 \mathrm{~m} / \mathrm{s}$ (continued) 


\section{UNCLASSIFIED}

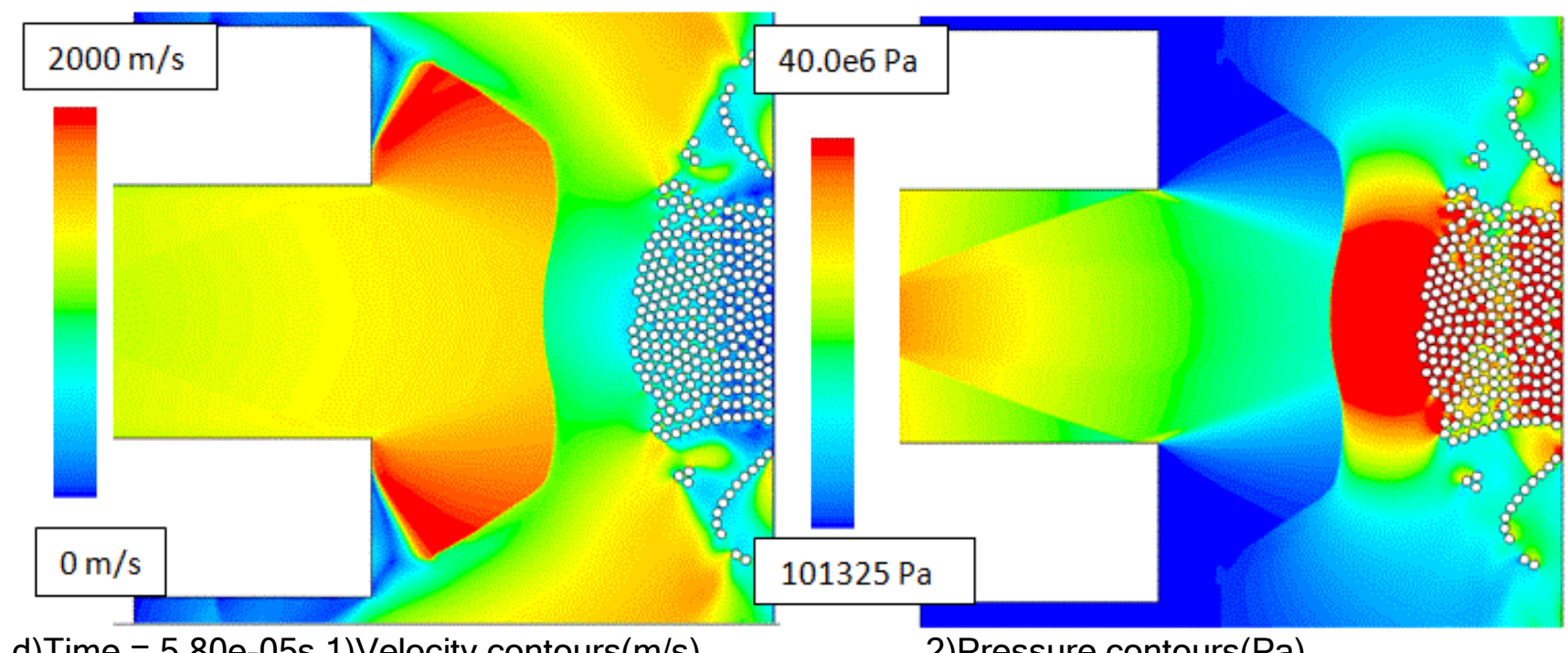

d) Time $=5.80 \mathrm{e}-05 \mathrm{~s} 1)$ Velocity contours $(\mathrm{m} / \mathrm{s})$

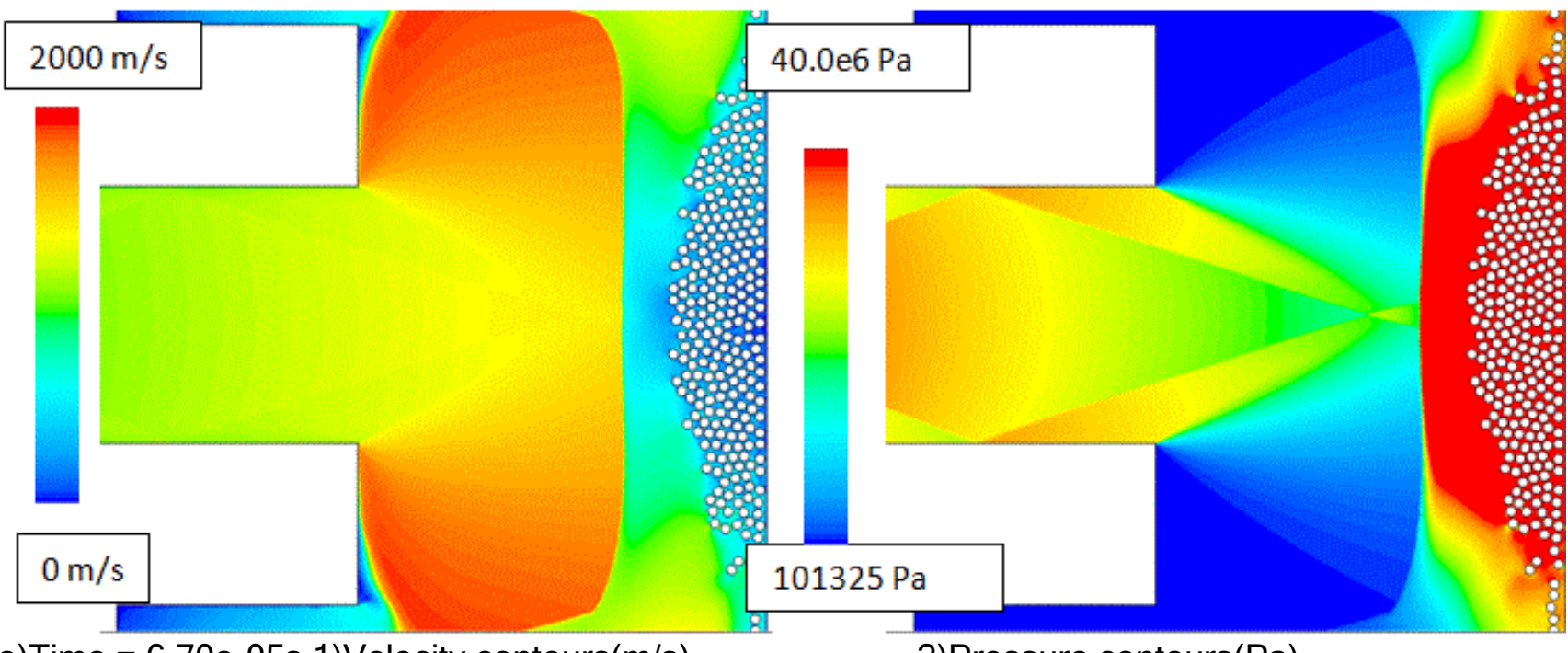

e)Time $=6.70 \mathrm{e}-05 \mathrm{~s} 1)$ Velocity contours $(\mathrm{m} / \mathrm{s})$

2)Pressure contours $(\mathrm{Pa})$

Figure 14. Velocity and pressure contours for case of $272300 \mu \mathrm{m}$ diameter particles, initial velocity of $250 \mathrm{~m} / \mathrm{s}$ - average impact velocity of $430 \mathrm{~m} / \mathrm{s}$

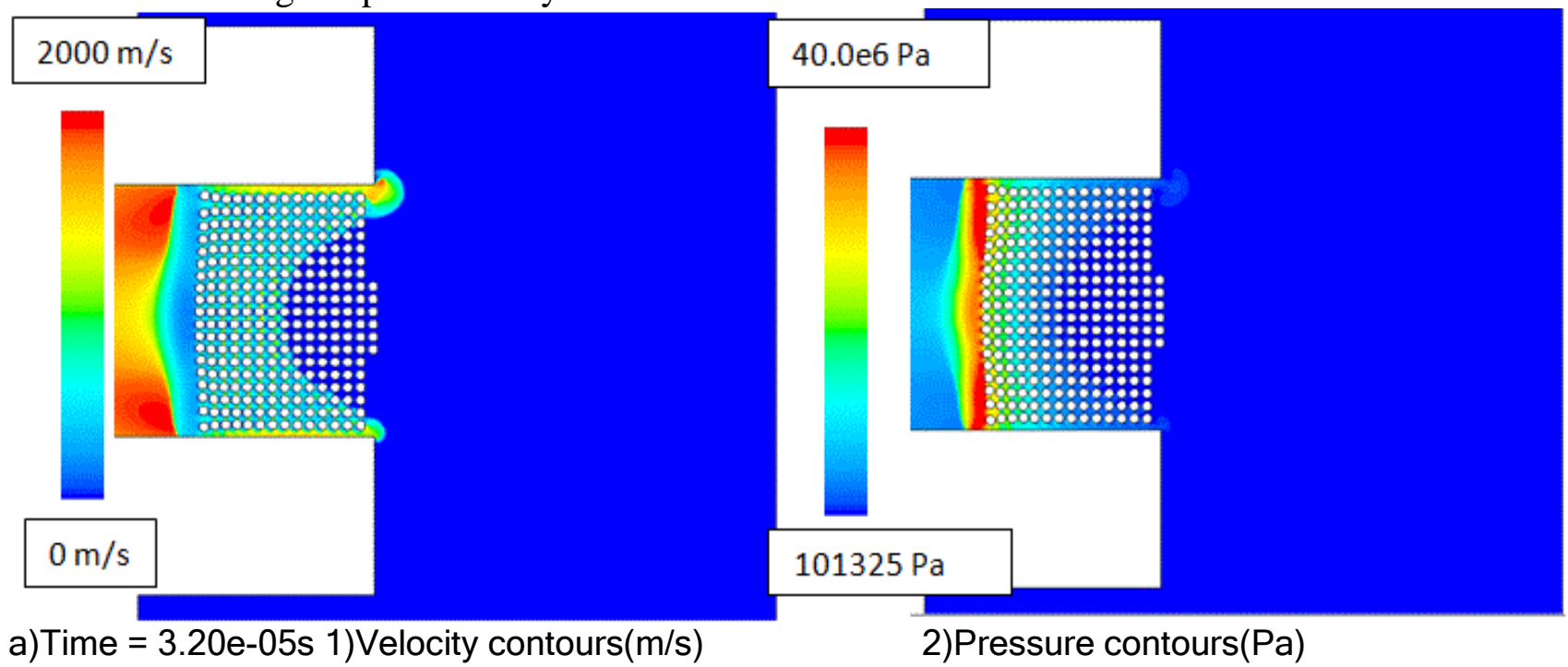




\section{UNCLASSIFIED}


Figure 15. Velocity and pressure contours for case of $272300 \mu \mathrm{m}$ diameter particles, initial velocity of $25 \mathrm{~m} / \mathrm{s}$ - average impact velocity of $530 \mathrm{~m} / \mathrm{s}$ (continued) 


\section{UNCLASSIFIED}

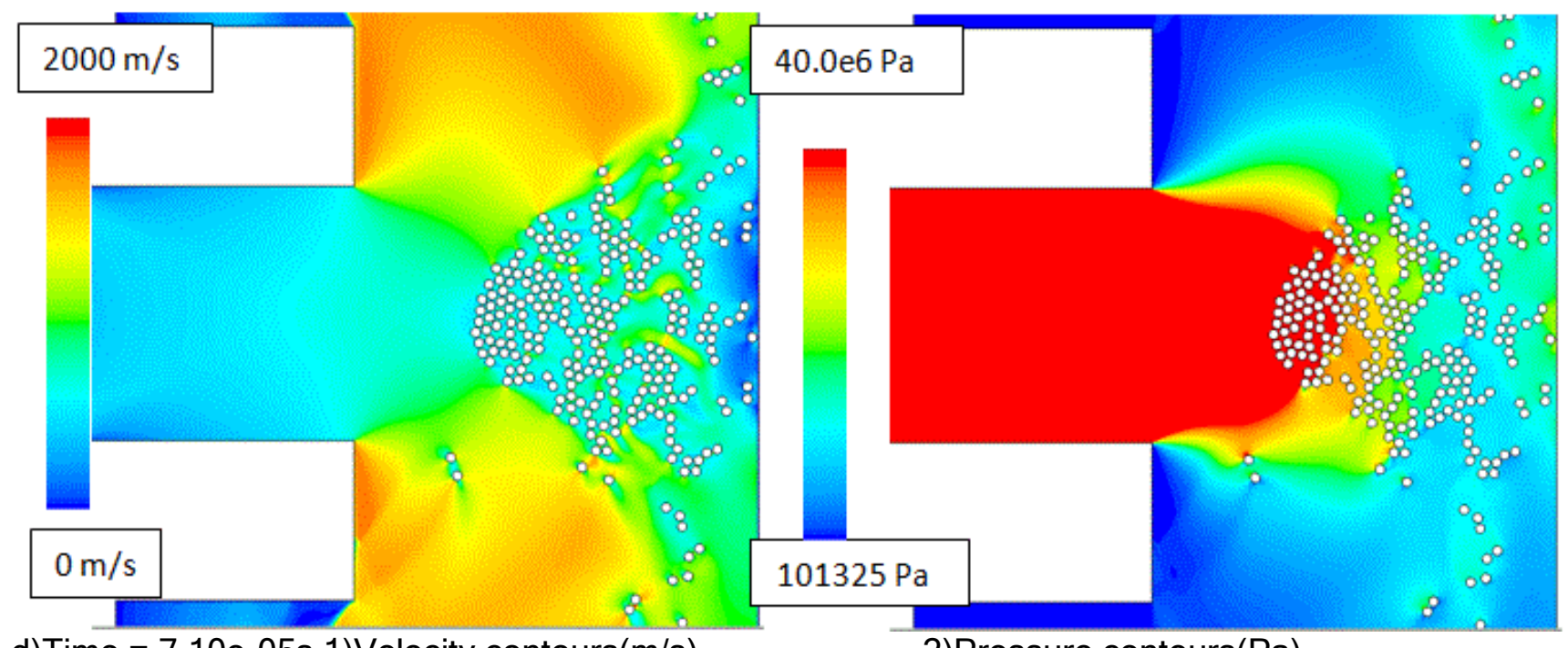

d)Time $=7.10 \mathrm{e}-05 \mathrm{~s}$ 1)Velocity contours $(\mathrm{m} / \mathrm{s})$

2)Pressure contours(Pa)

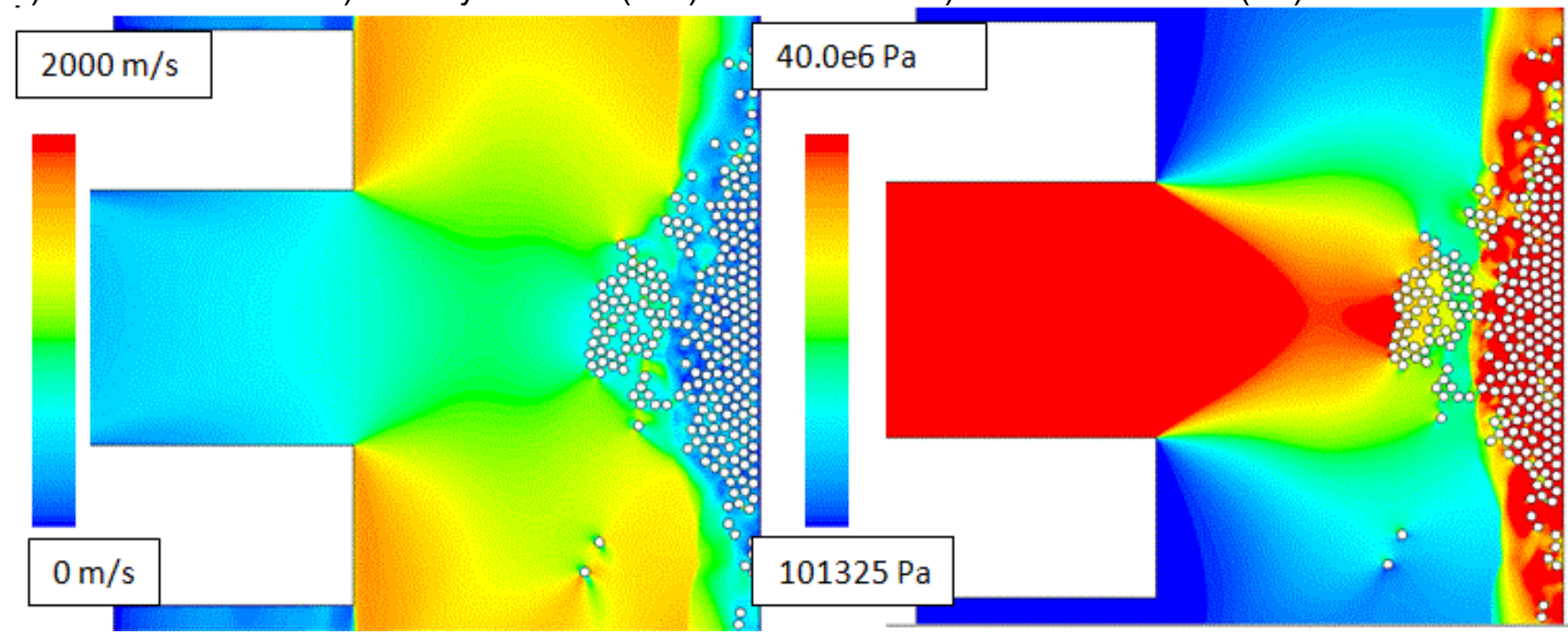

e) Time $=7.85 \mathrm{e}-05 \mathrm{~s} 1)$ Velocity contours $(\mathrm{m} / \mathrm{s})$

2)Pressure contours $(\mathrm{Pa})$

Figure 15. Velocity and pressure contours for case of $272300 \mu \mathrm{m}$ diameter particles, initial velocity of $25 \mathrm{~m} / \mathrm{s}$ - average impact velocity of $530 \mathrm{~m} / \mathrm{s}$ 


\section{UNCLASSIFIED}

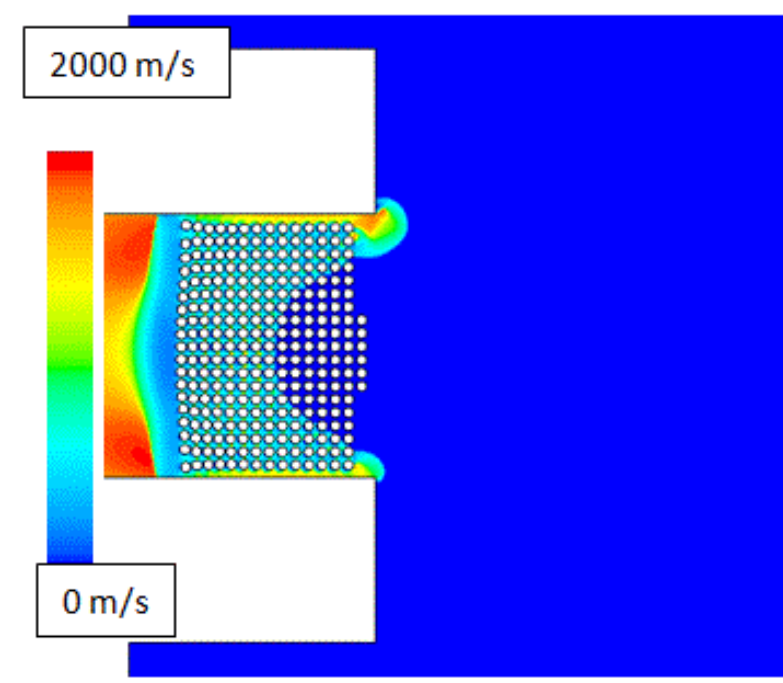

a)Time $=3.20 \mathrm{e}-05 \mathrm{~s} 1)$ Velocity contours $(\mathrm{m} / \mathrm{s})$
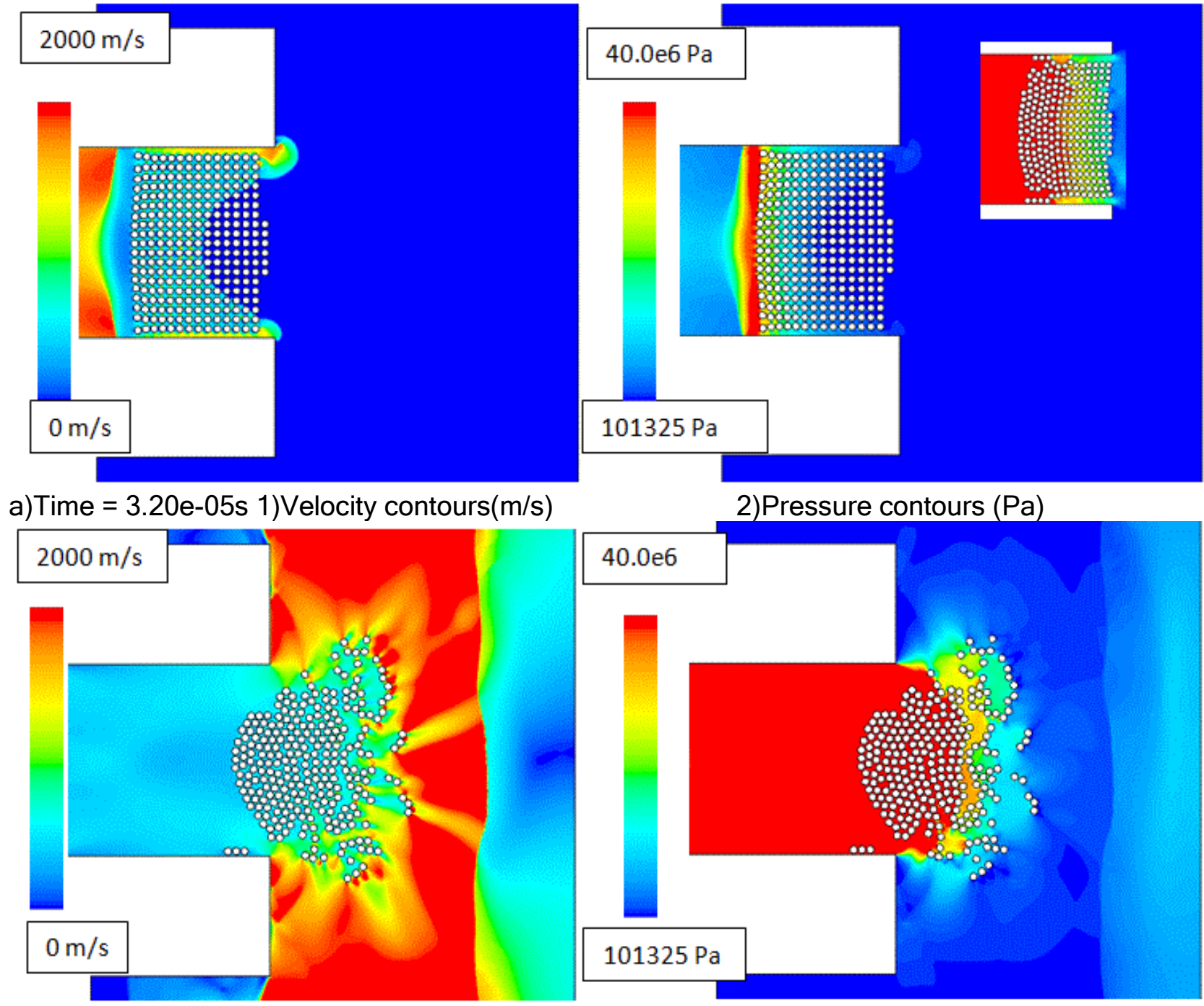

b) Time $=5.80 \mathrm{e}-05 \mathrm{~s} 1)$ Velocity contours $(\mathrm{m} / \mathrm{s})$
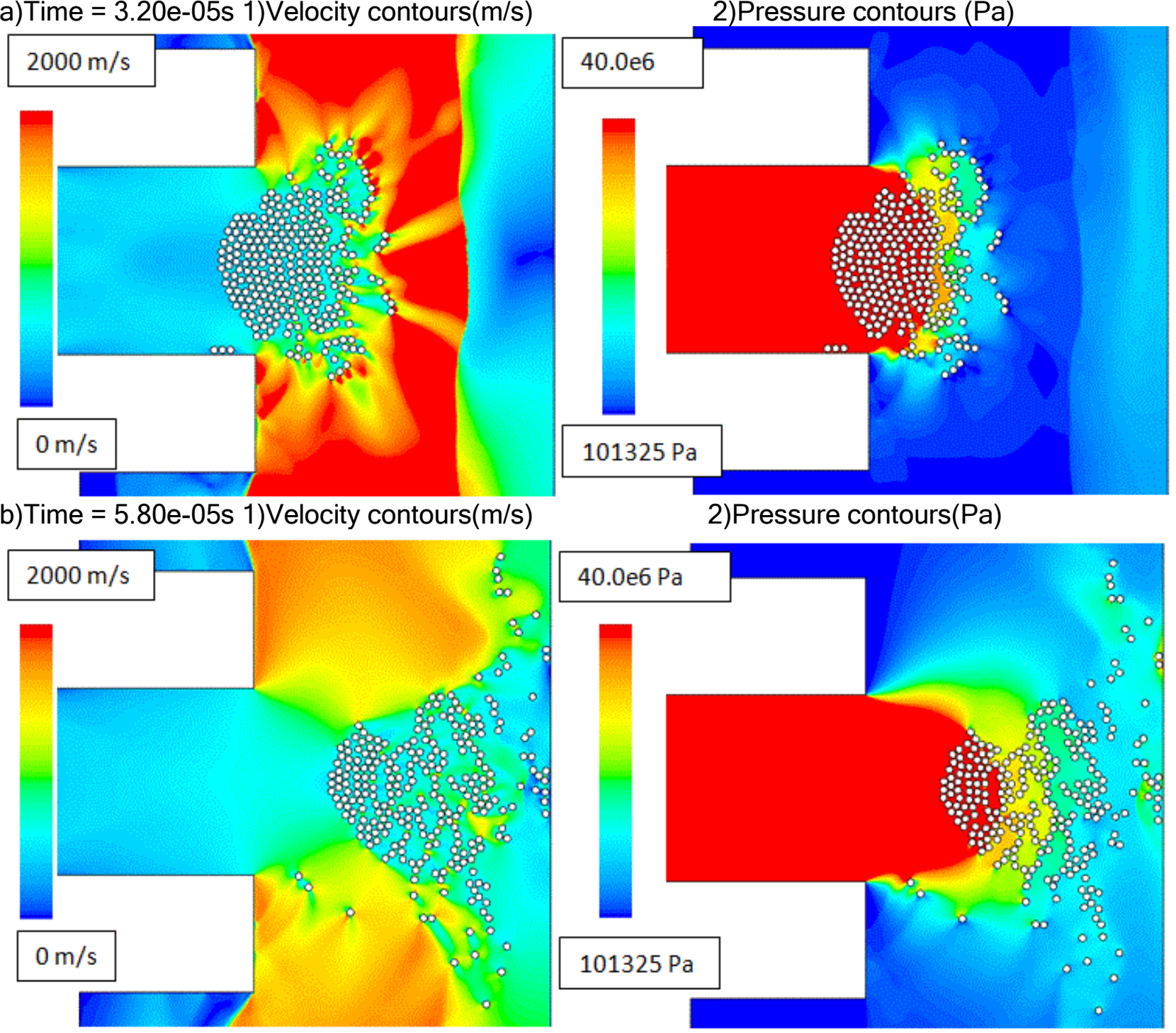

c) Time $=7.10 \mathrm{e}-05 \mathrm{~s} 1)$ Velocity contours $(\mathrm{m} / \mathrm{s})$

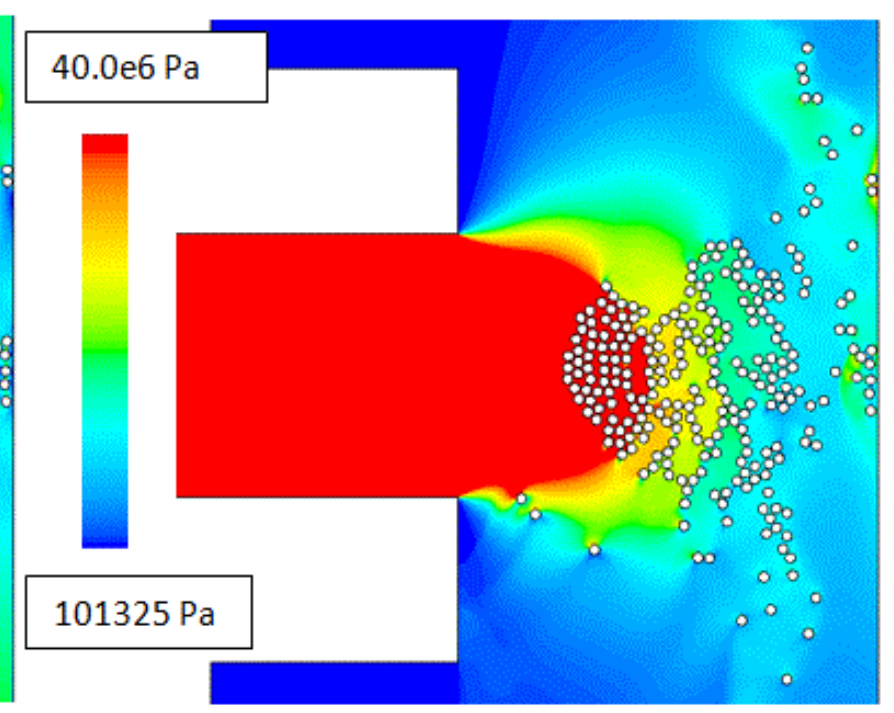

2)Pressure contours $(\mathrm{Pa})$

Figure 16. Velocity and pressure contours for case of $272300 \mu \mathrm{m}$ diameter particles, initial velocity of $10 \mathrm{~m} / \mathrm{s}$ - average impact velocity of $620 \mathrm{~m} / \mathrm{s}$ (continued) 


\section{UNCLASSIFIED}

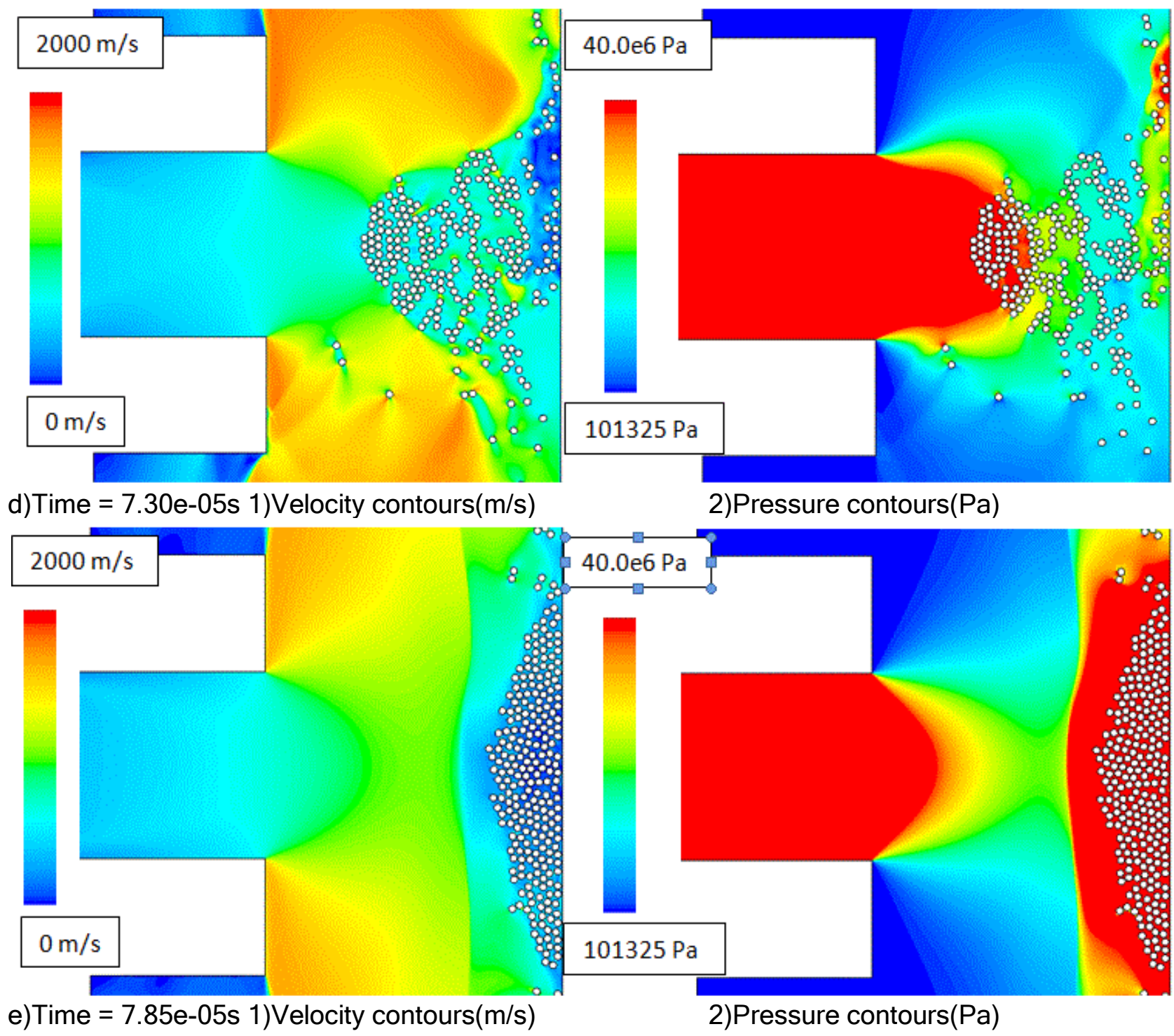

Figure 16. Velocity and pressure contours for case of $272300 \mu \mathrm{m}$ diameter particles, initial velocity of $10 \mathrm{~m} / \mathrm{s}$ - average impact velocity of $620 \mathrm{~m} / \mathrm{s}$ 


\section{UNCLASSIFIED}

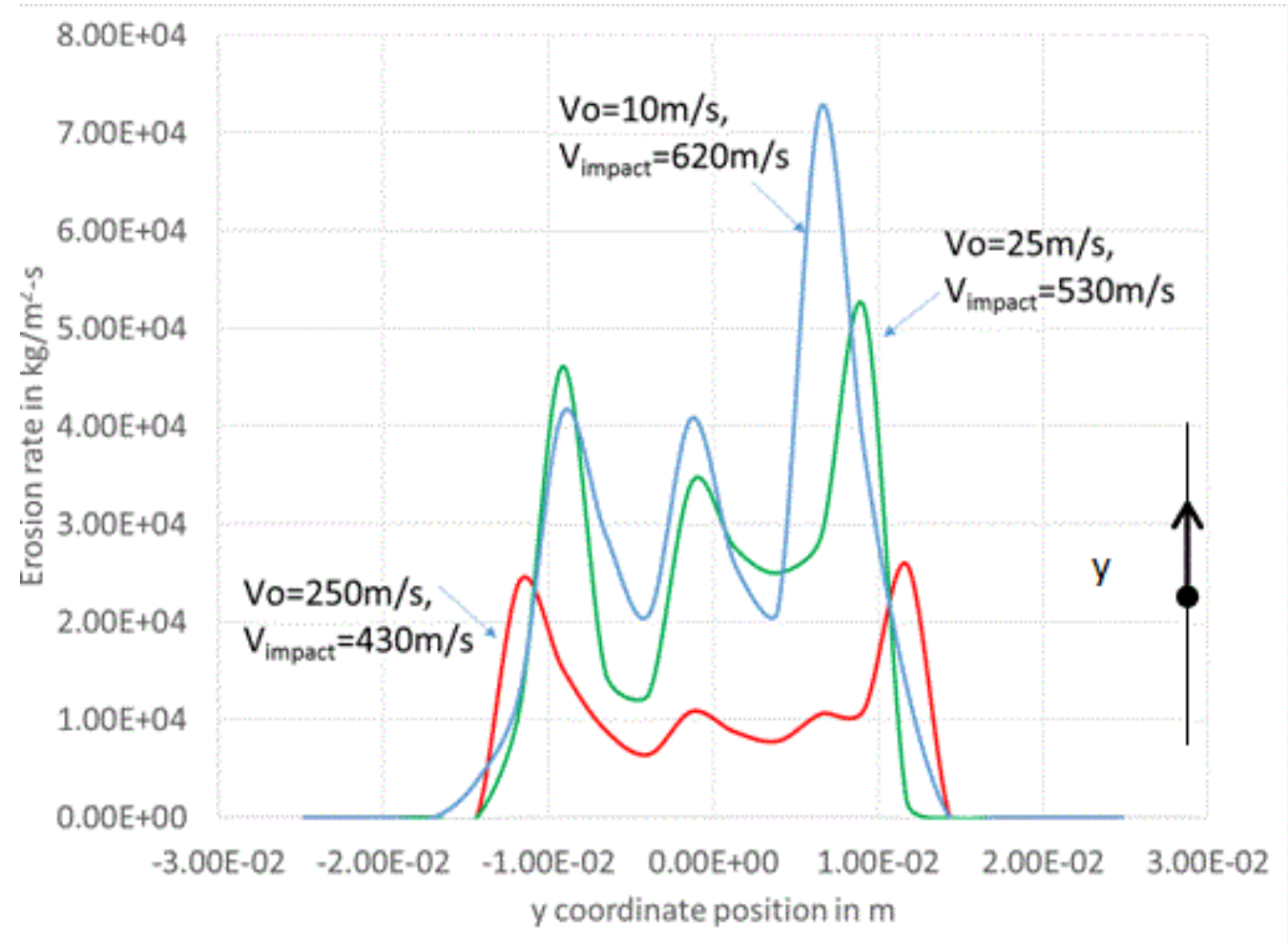

Figure 17. Erosion distribution at 9.0e-05 seconds for indicated particle velocity cases

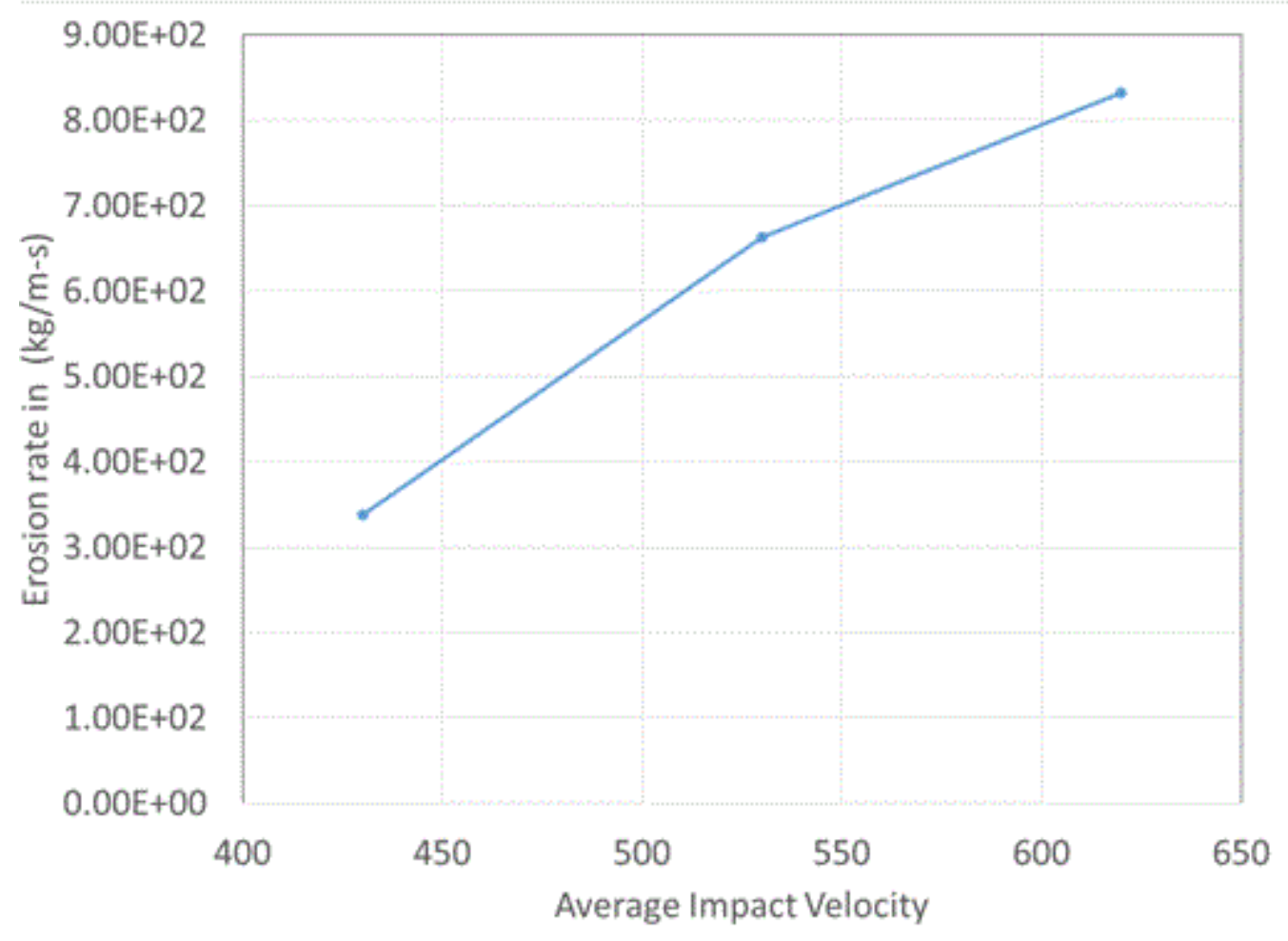

Figure 18. Net erosion rate as a function of the average impact velocity 


\section{UNCLASSIFIED}

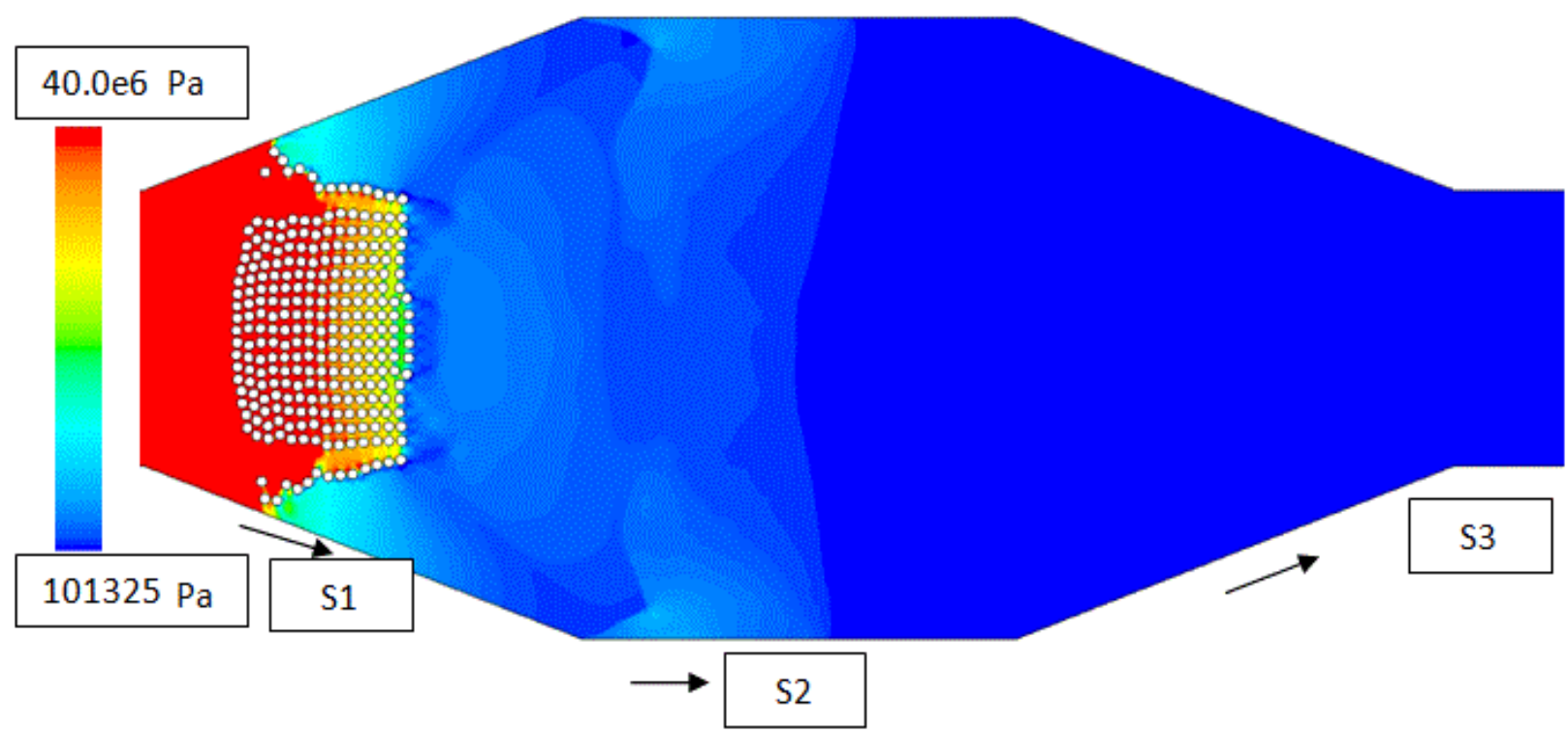

a) Time $=5.74 \mathrm{e}-05 \mathrm{~s}$

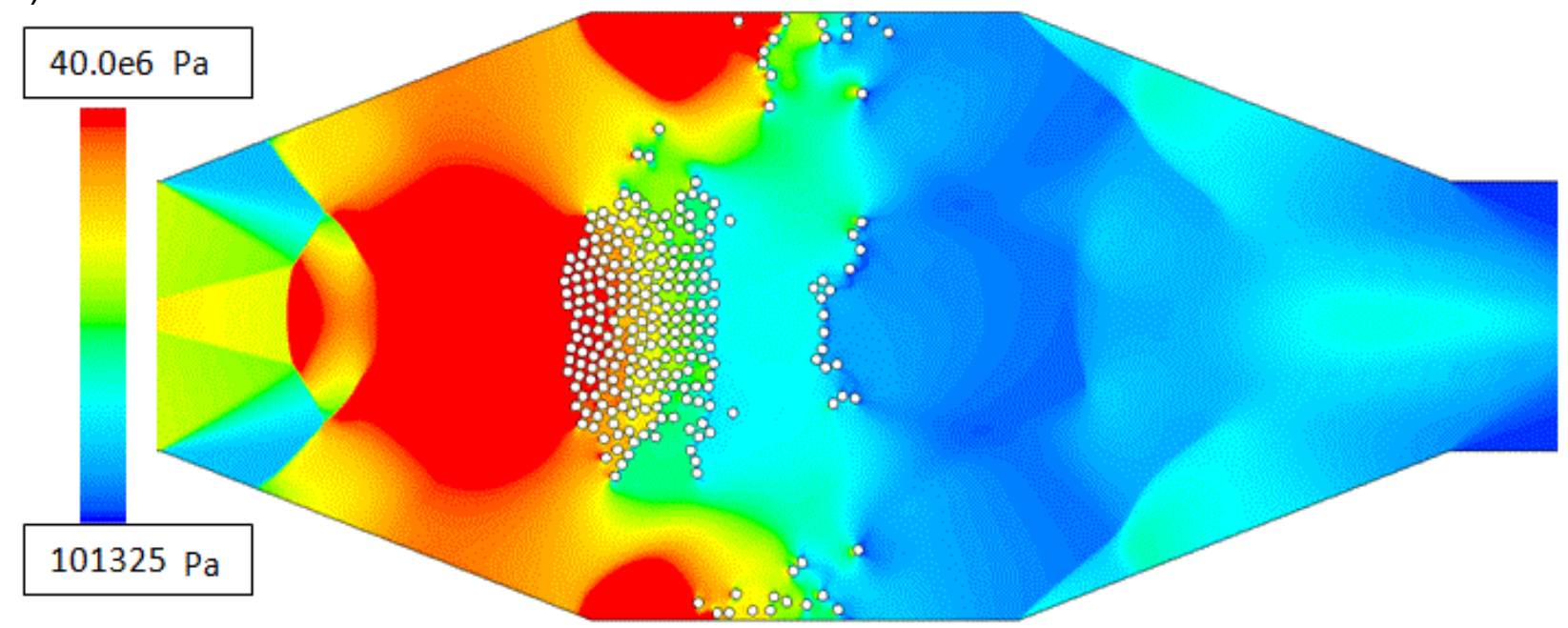

b) Time $=8.38 \mathrm{e}-05 \mathrm{~s}$



c) Time $=1.03 \mathrm{e}-04 \mathrm{~s}$

Figure 19. Pressure contours (Pa) for diverging/converging section(continued) 


\section{UNCLASSIFIED}

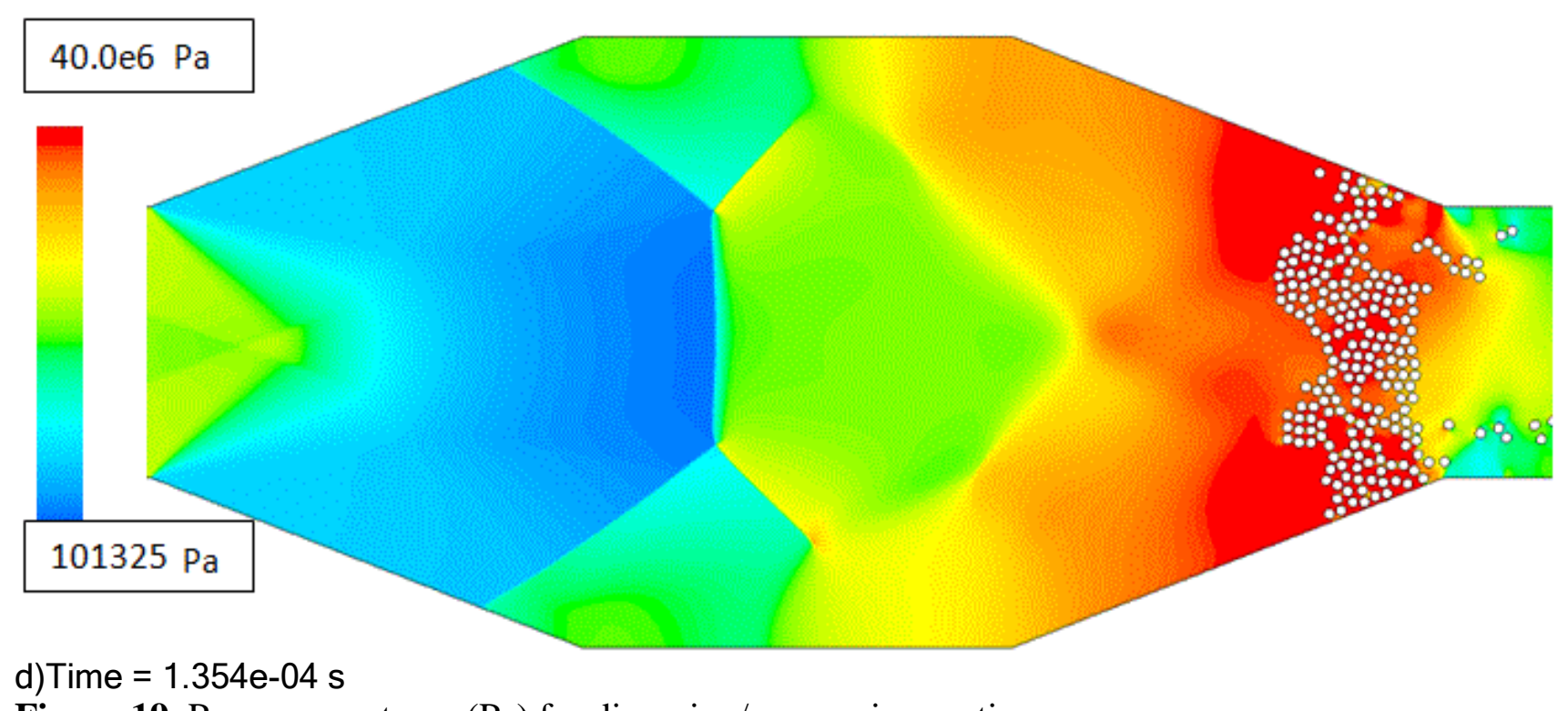

Figure 19. Pressure contours $(\mathrm{Pa})$ for diverging/converging section

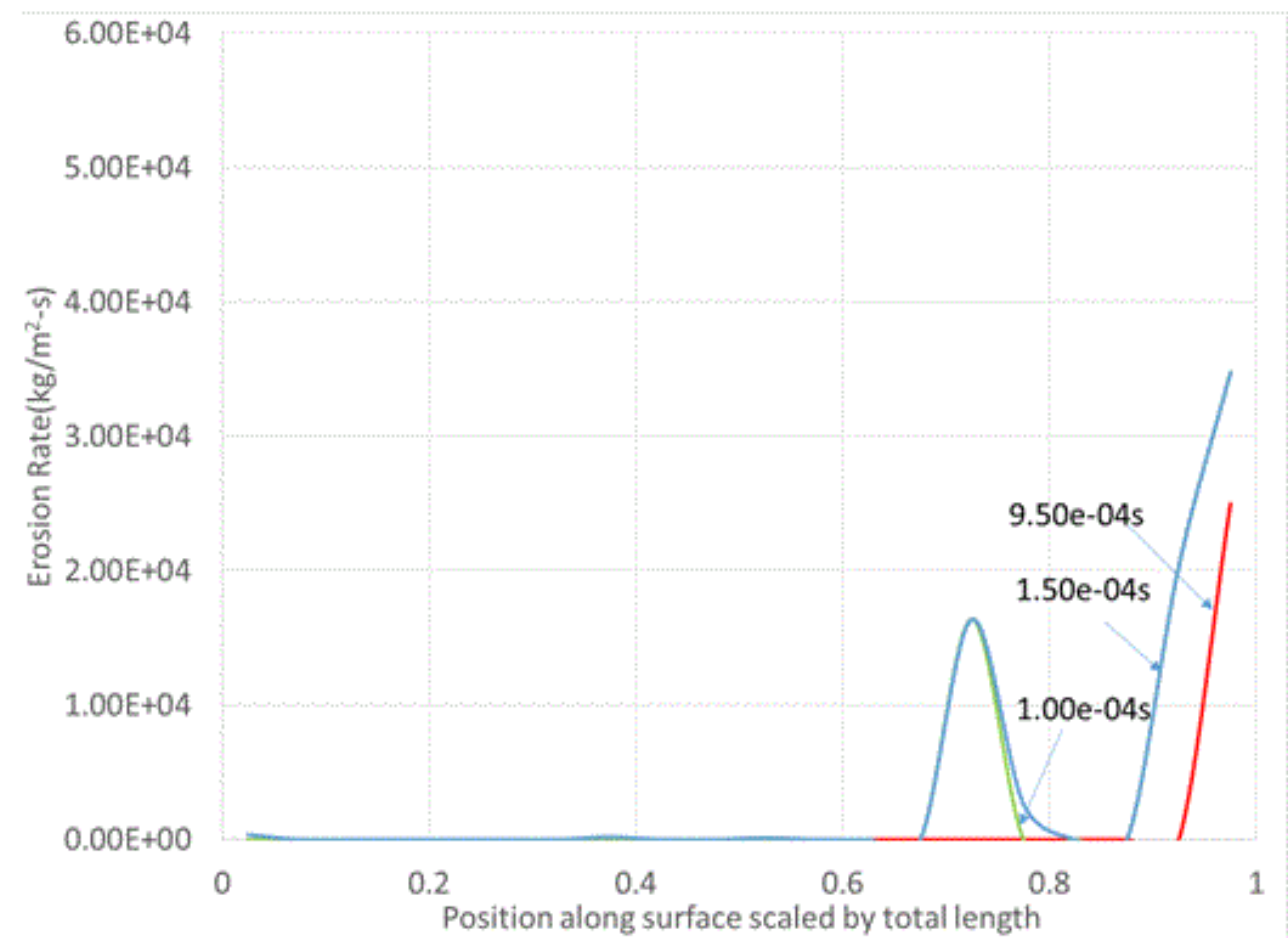

a)horizontal(surface S2 in figure $2 \mathrm{a}$ )

Figure 20. Erosion rate distributions along indicated surfaces for diverging/converging section(continued) 


\section{UNCLASSIFIED}

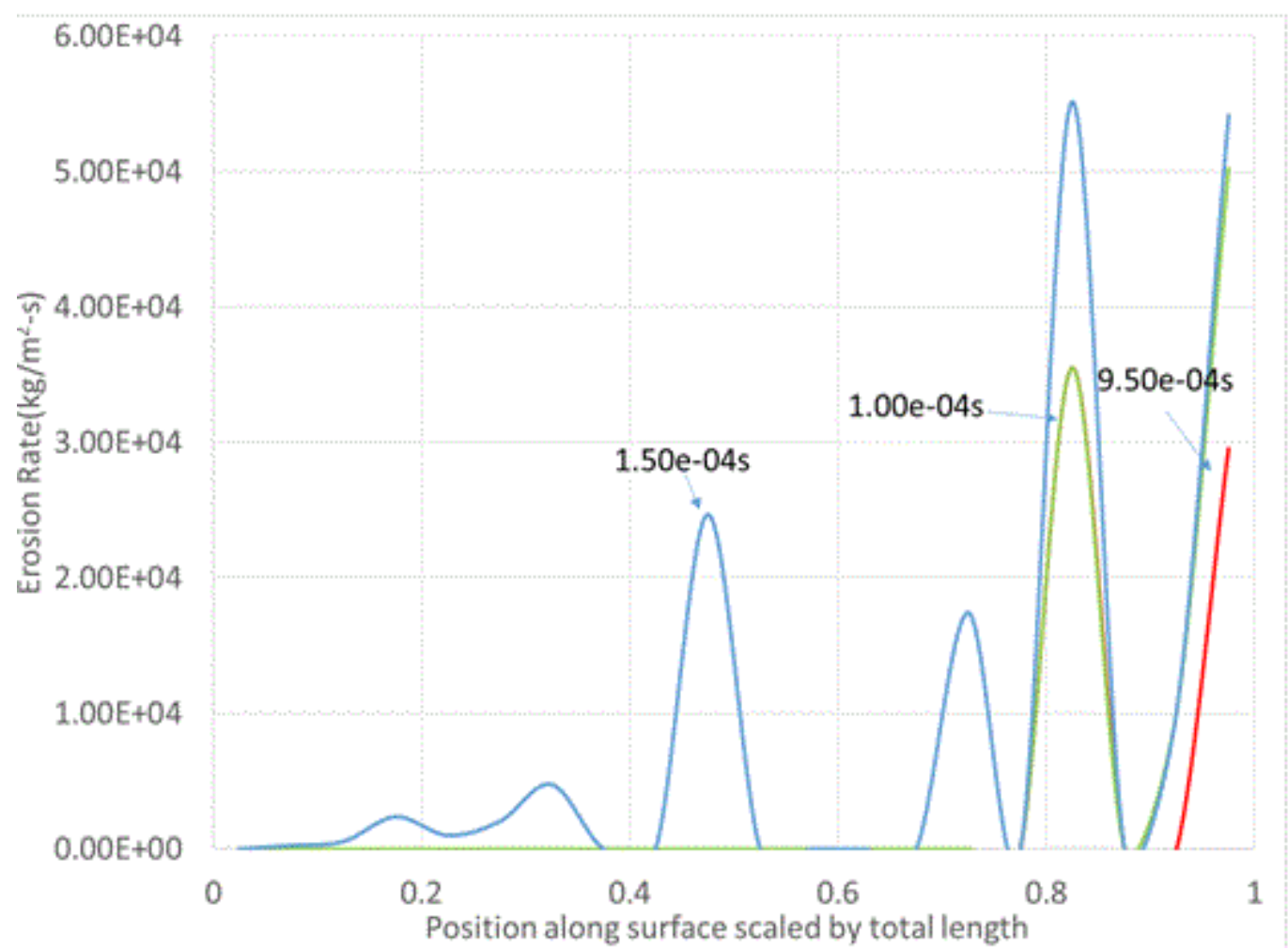

b)converging (surface $\mathrm{S} 3$ in figure $2 \mathrm{a}$ )

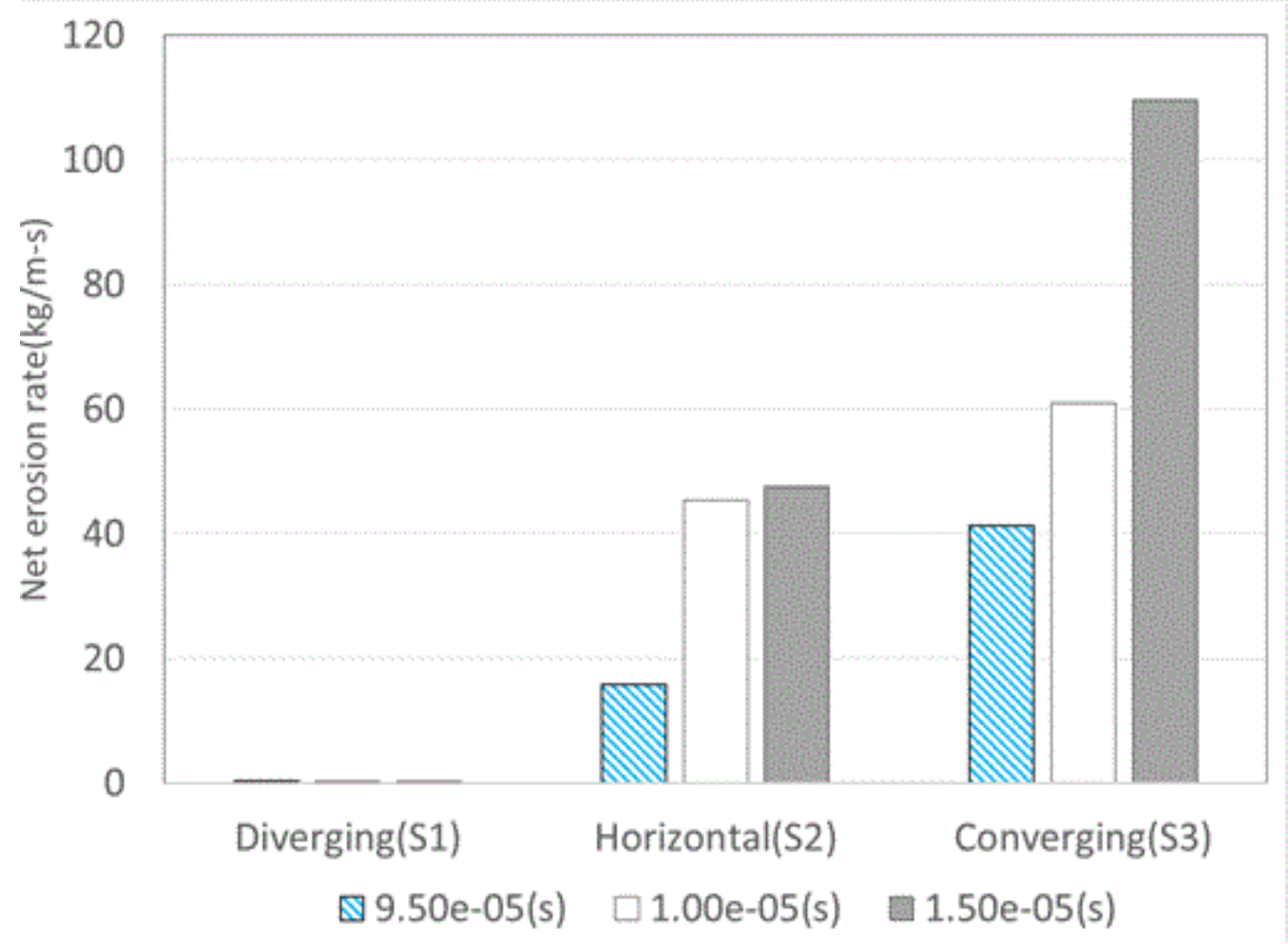

c)Net erosion rate development

Figure 20. Erosion rate distributions along indicated surfaces for diverging/converging section 


\section{UNCLASSIFIED}

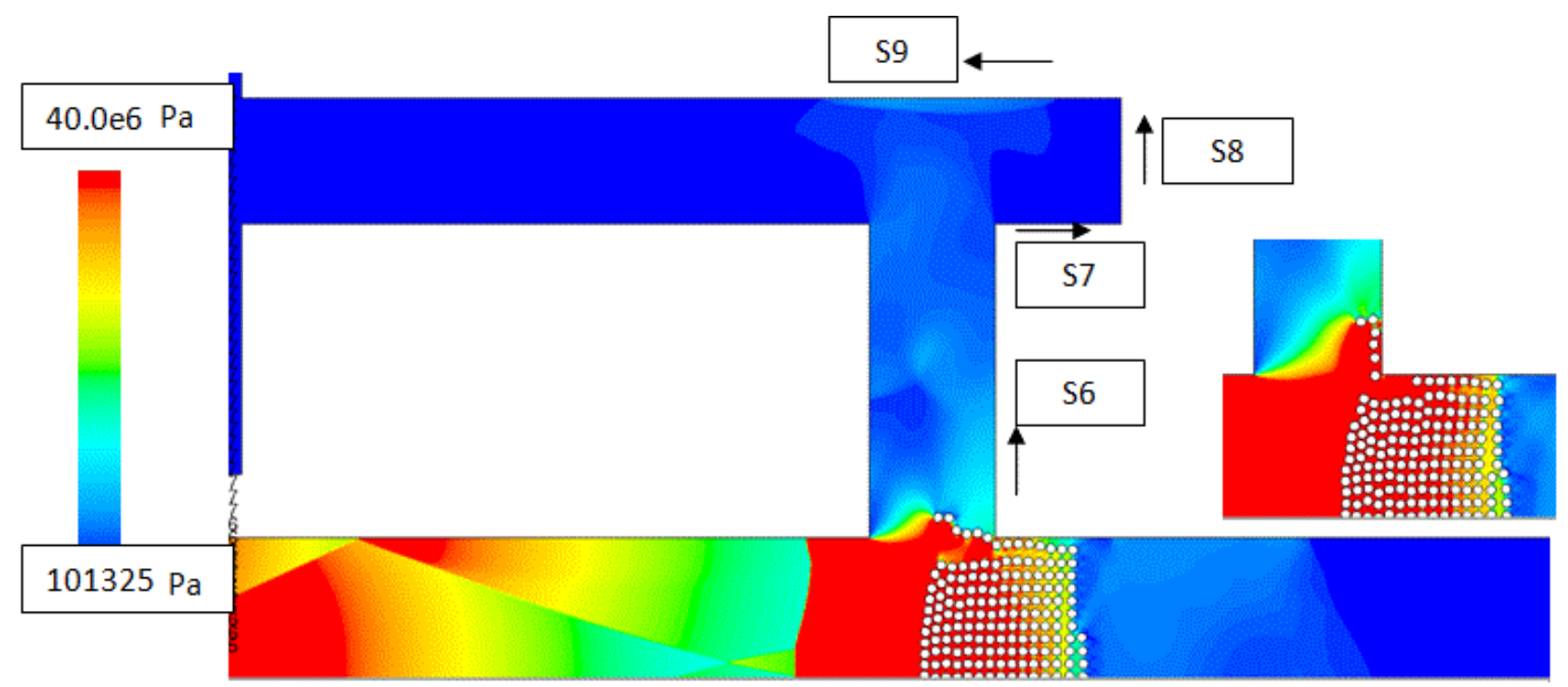

a) Time $=5.44 \mathrm{e}-05 \mathrm{~s}$

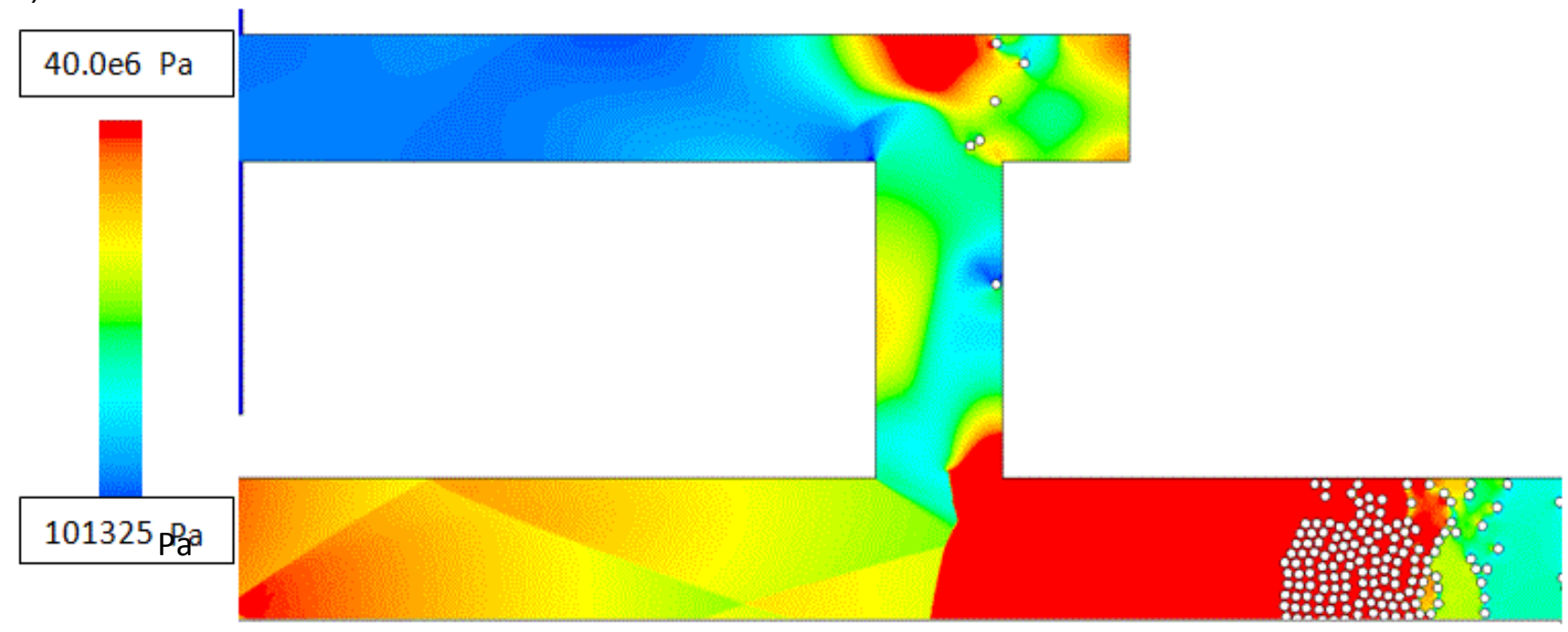

b) Time $=8.68 \mathrm{e}-05 \mathrm{~s}$

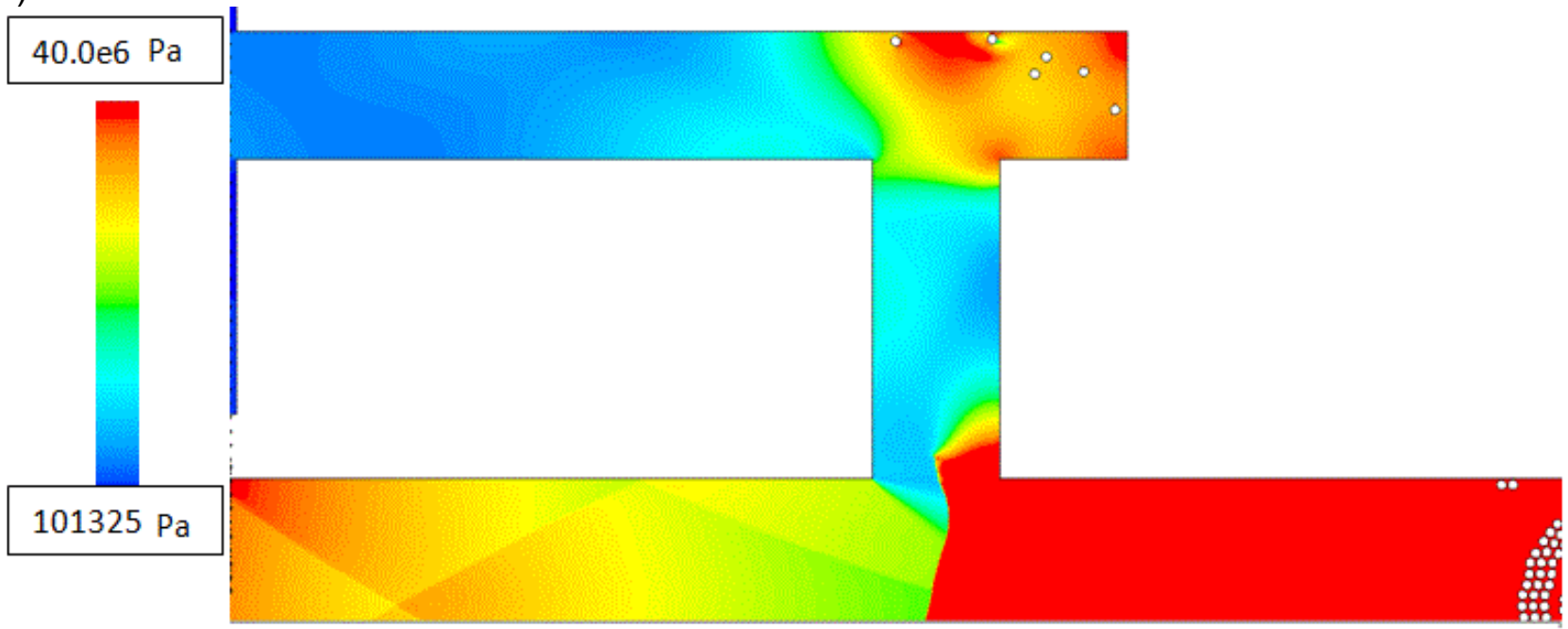

c) Time $=1.06 \mathrm{e}-04 \mathrm{~s}$

Figure 21. Pressure contours $(\mathrm{Pa})$ for by-pass configuration(continued) 


\section{UNCLASSIFIED}

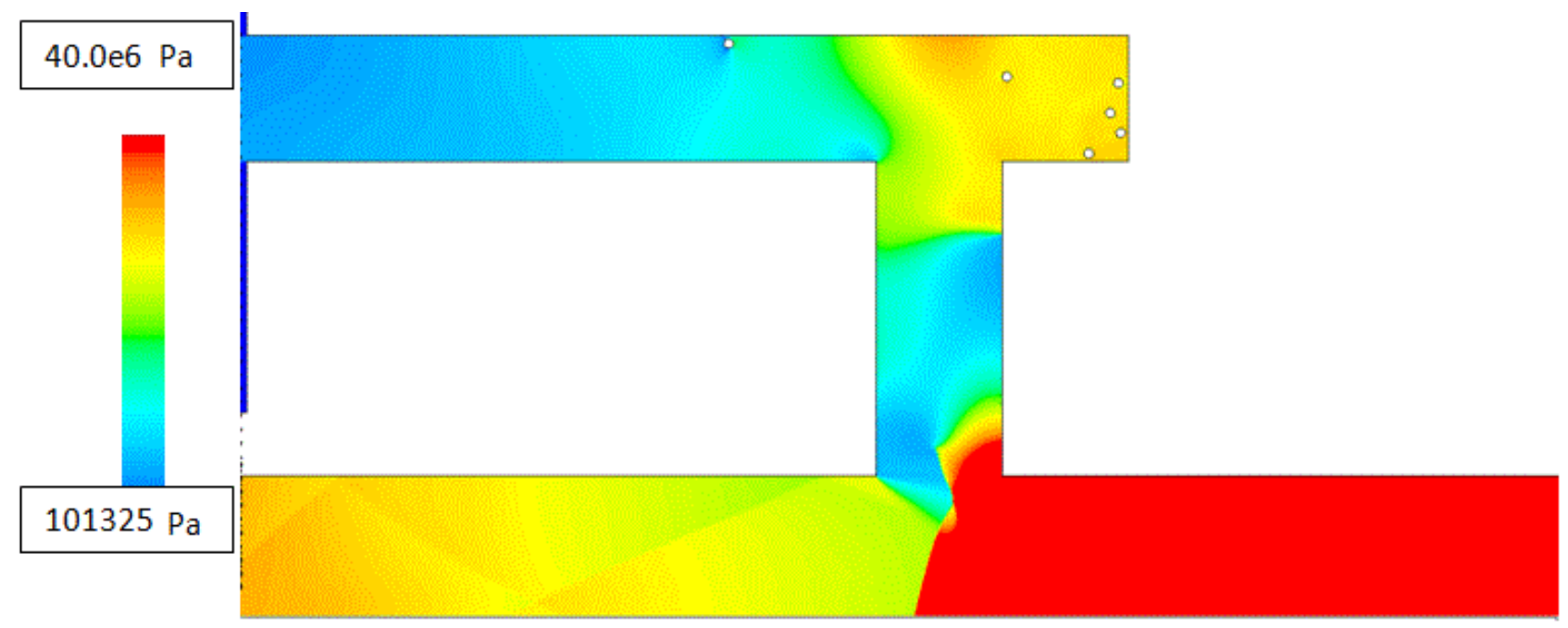

d) Time $=1.27 \mathrm{e}-04 \mathrm{~s}$

Figure 21. Pressure contours $(\mathrm{Pa})$ for by-pass configuration

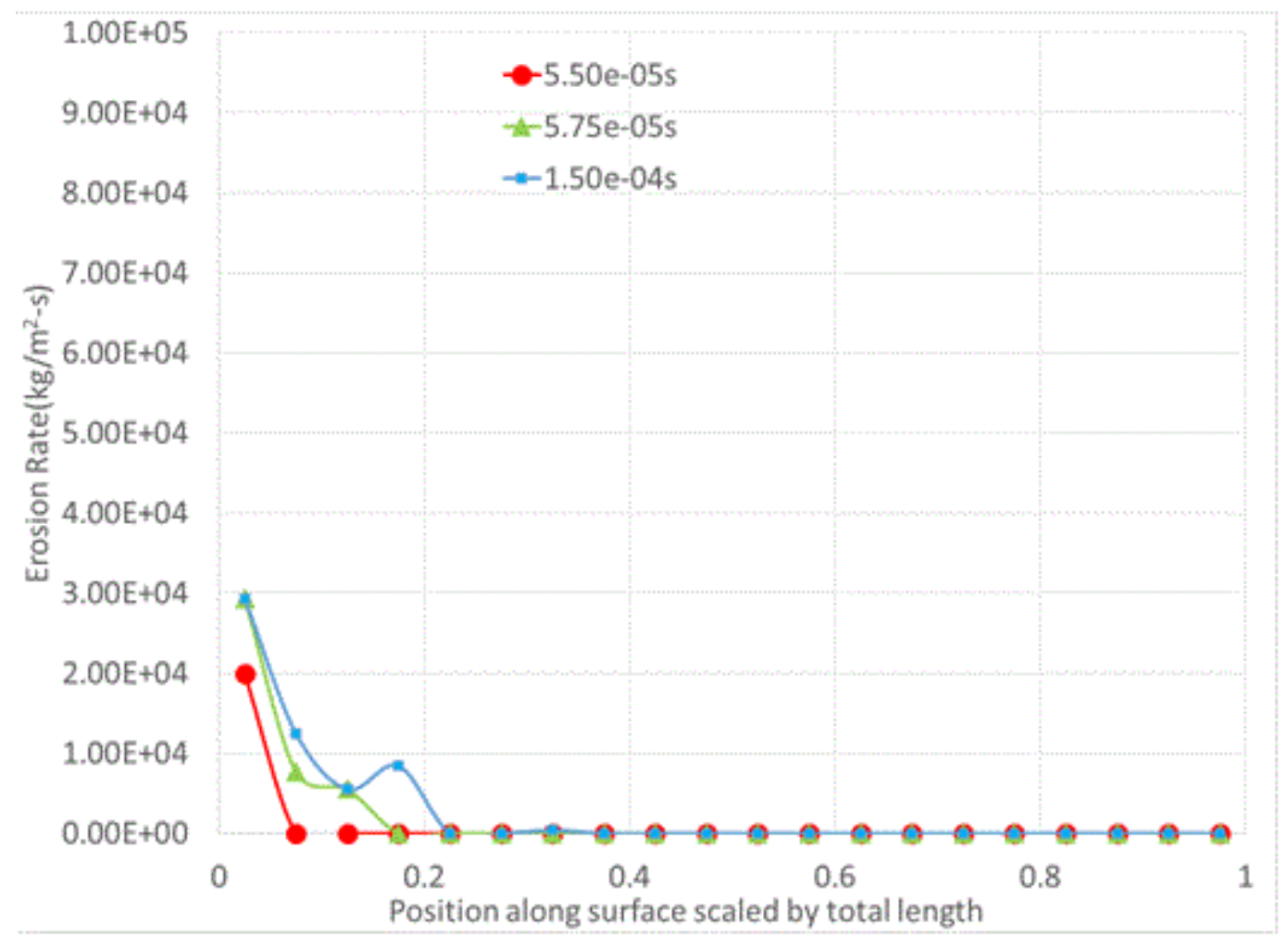

a)surface $S 6$

Figure 22. Erosion rate distributions along indicated surfaces for by-pass configuration(continued) 


\section{UNCLASSIFIED}

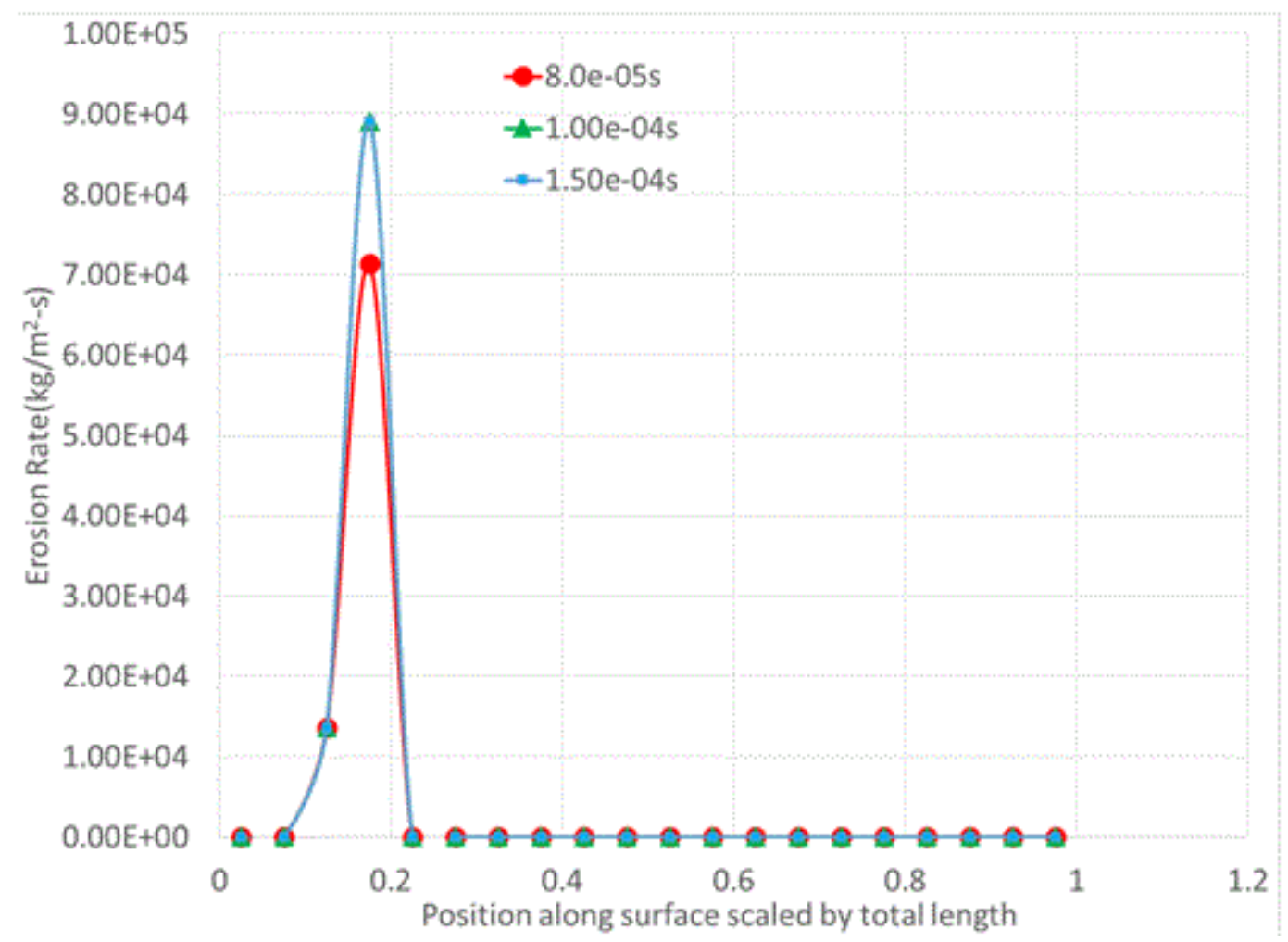

b)surface $S 9$

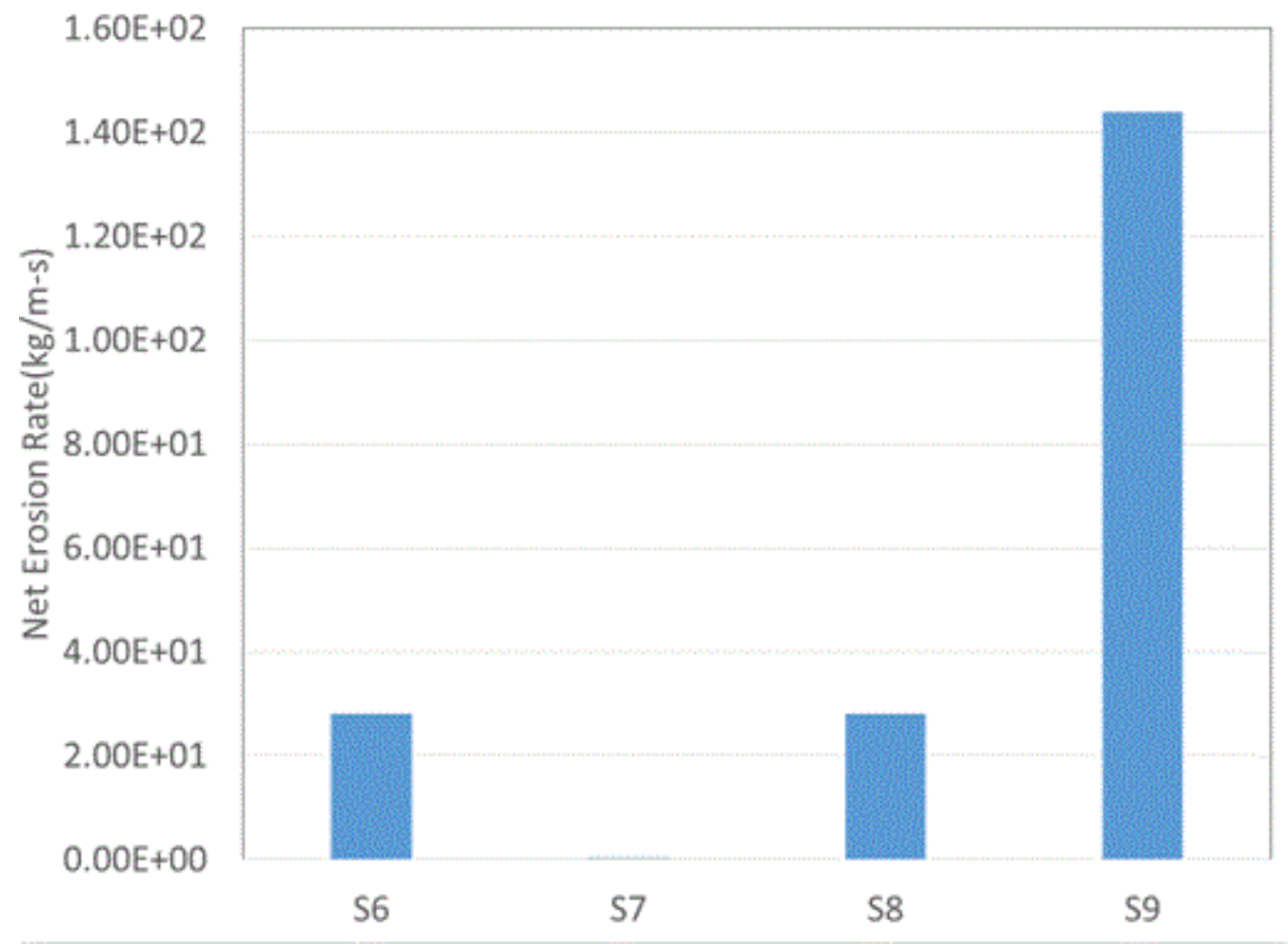

c)Net erosion rate development

Figure 22. Erosion rate distributions along indicated surfaces for by-pass configuration 


\section{UNCLASSIFIED}

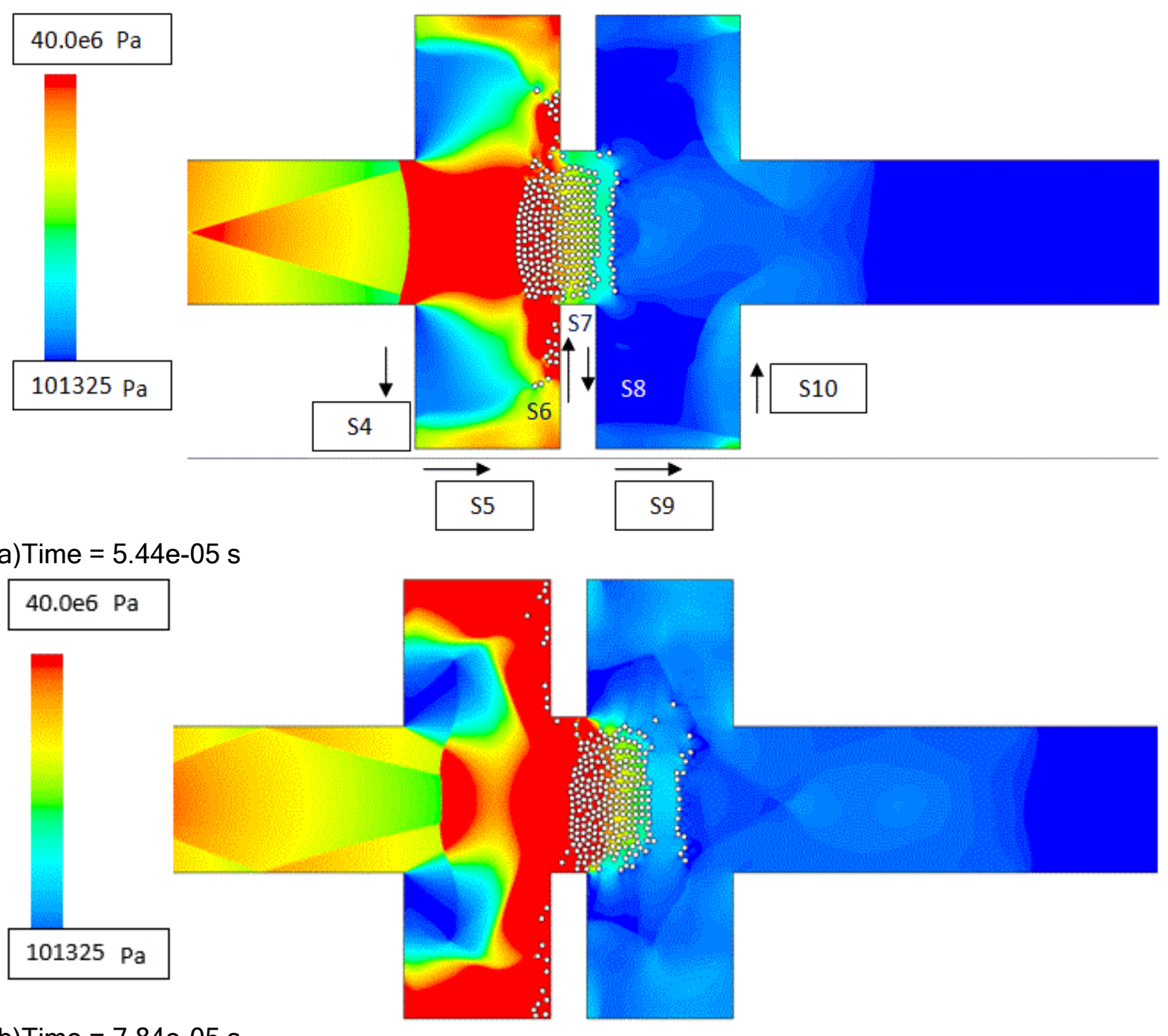

b) Time $=7.84 \mathrm{e}-05 \mathrm{~s}$

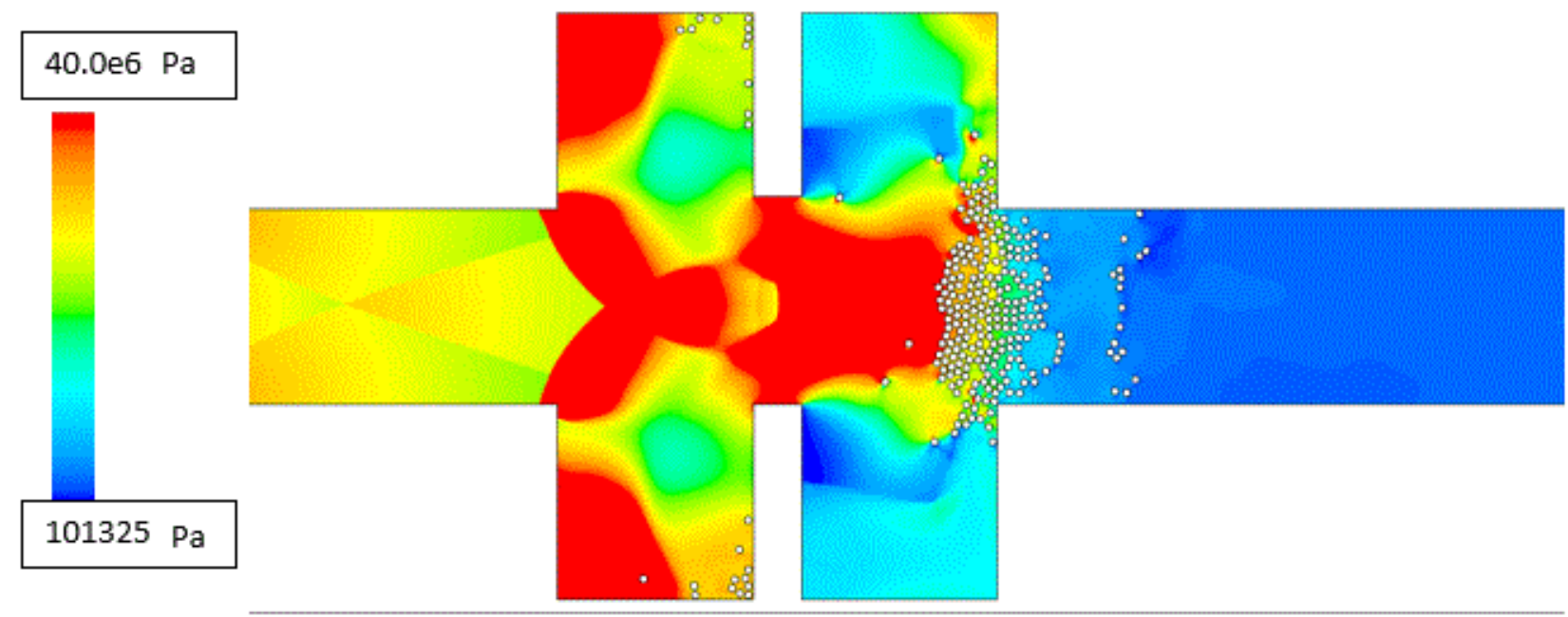

c) Time $=8.68 \mathrm{e}-05 \mathrm{~s}$

Figure 23. Pressure contours $(\mathrm{Pa})$ for baffle configuration(continued) 


\section{UNCLASSIFIED}

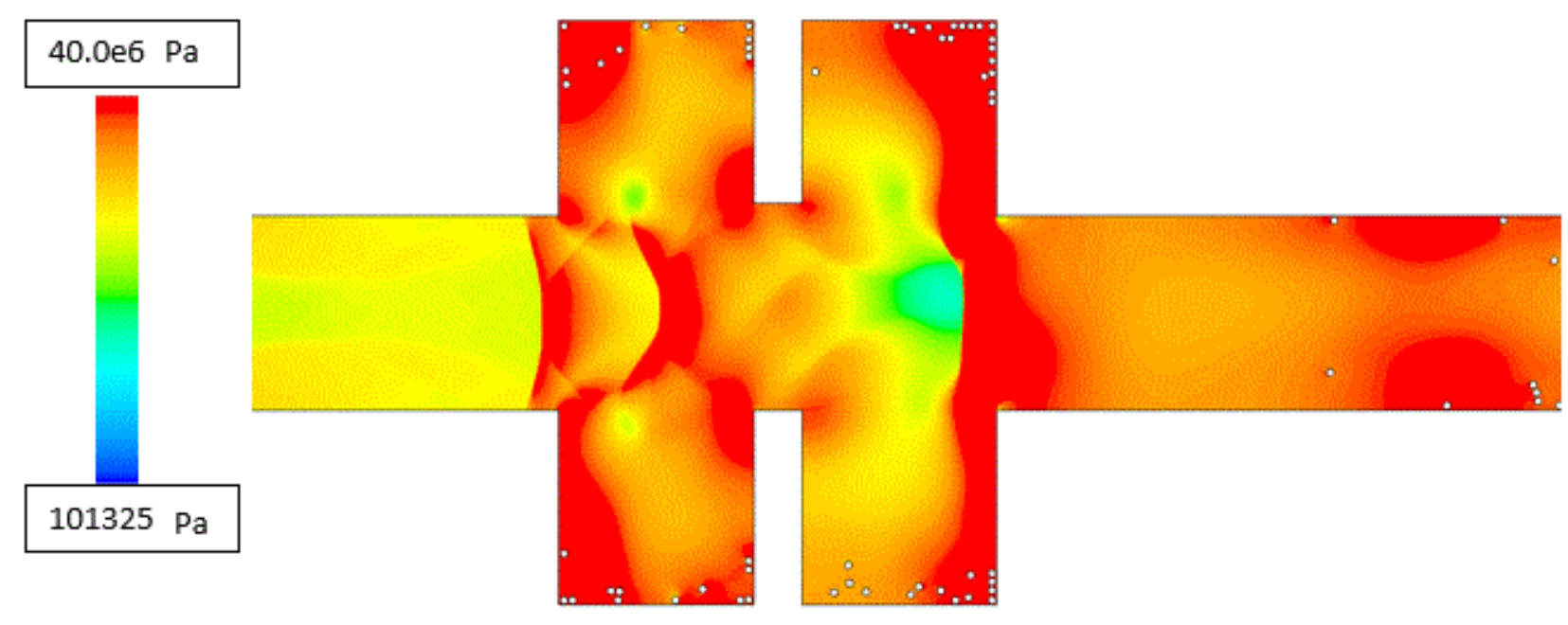

d) Time $=1.58 \mathrm{e}-04 \mathrm{~s}$

Figure 23. Pressure contours $(\mathrm{Pa})$ for baffle configuration

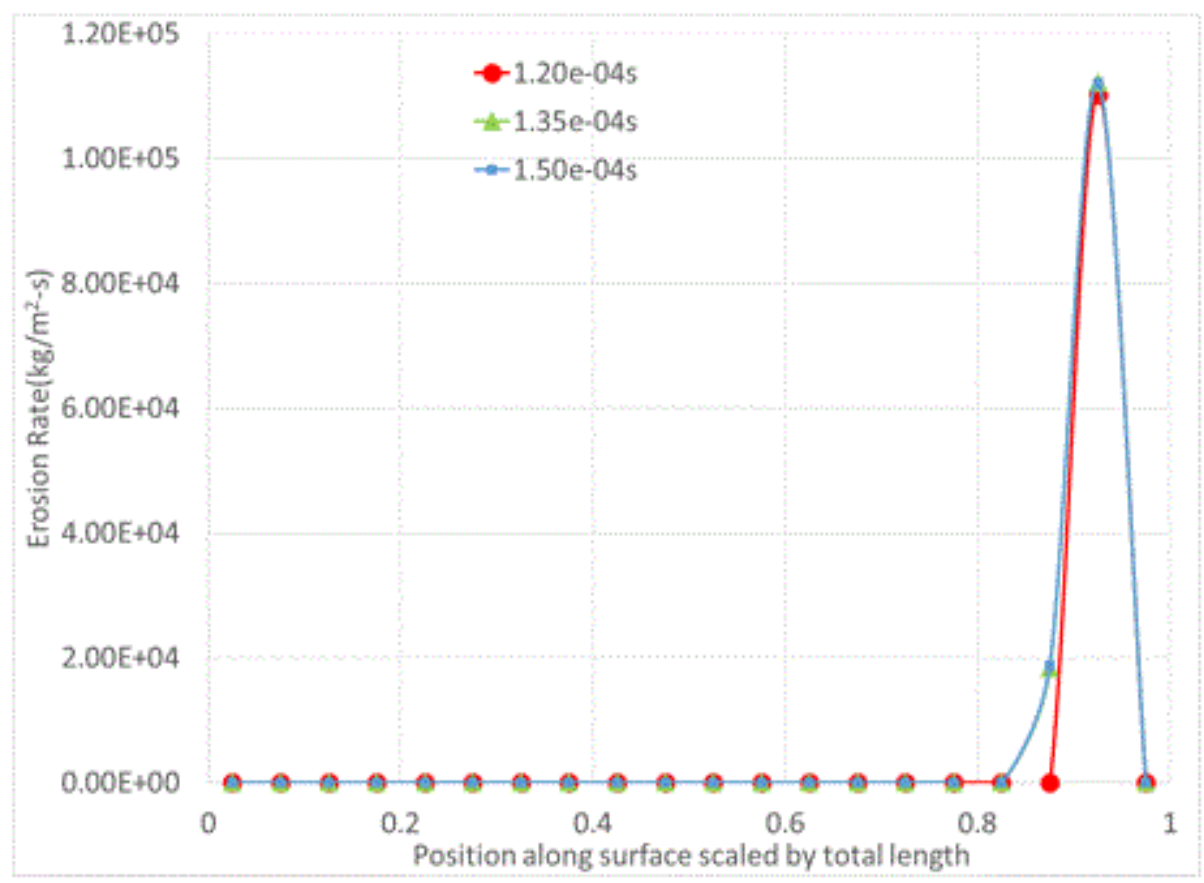

a)surface $\mathrm{S} 5$

Figure 24. Erosion rate distributions along indicated surfaces for baffle configuration(continued) 


\section{UNCLASSIFIED}

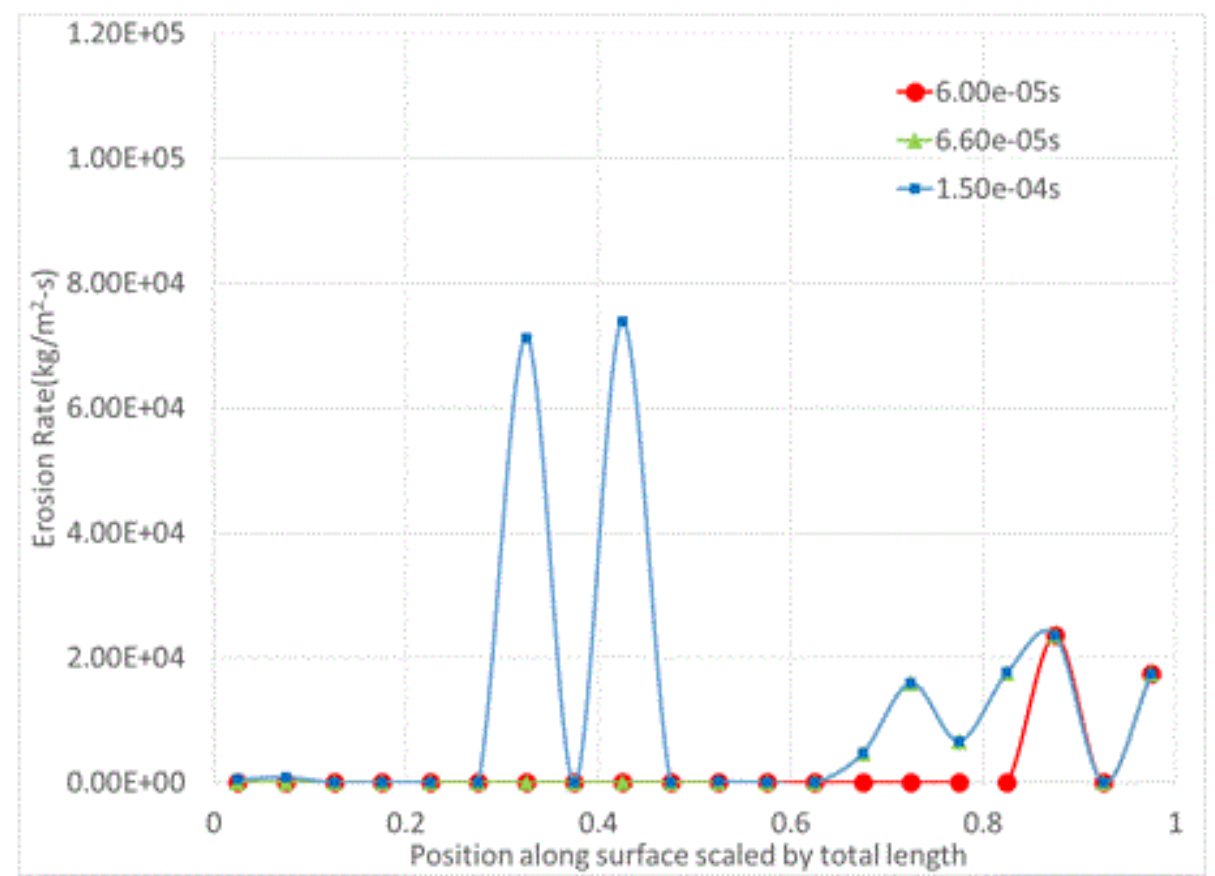

b)surface S6

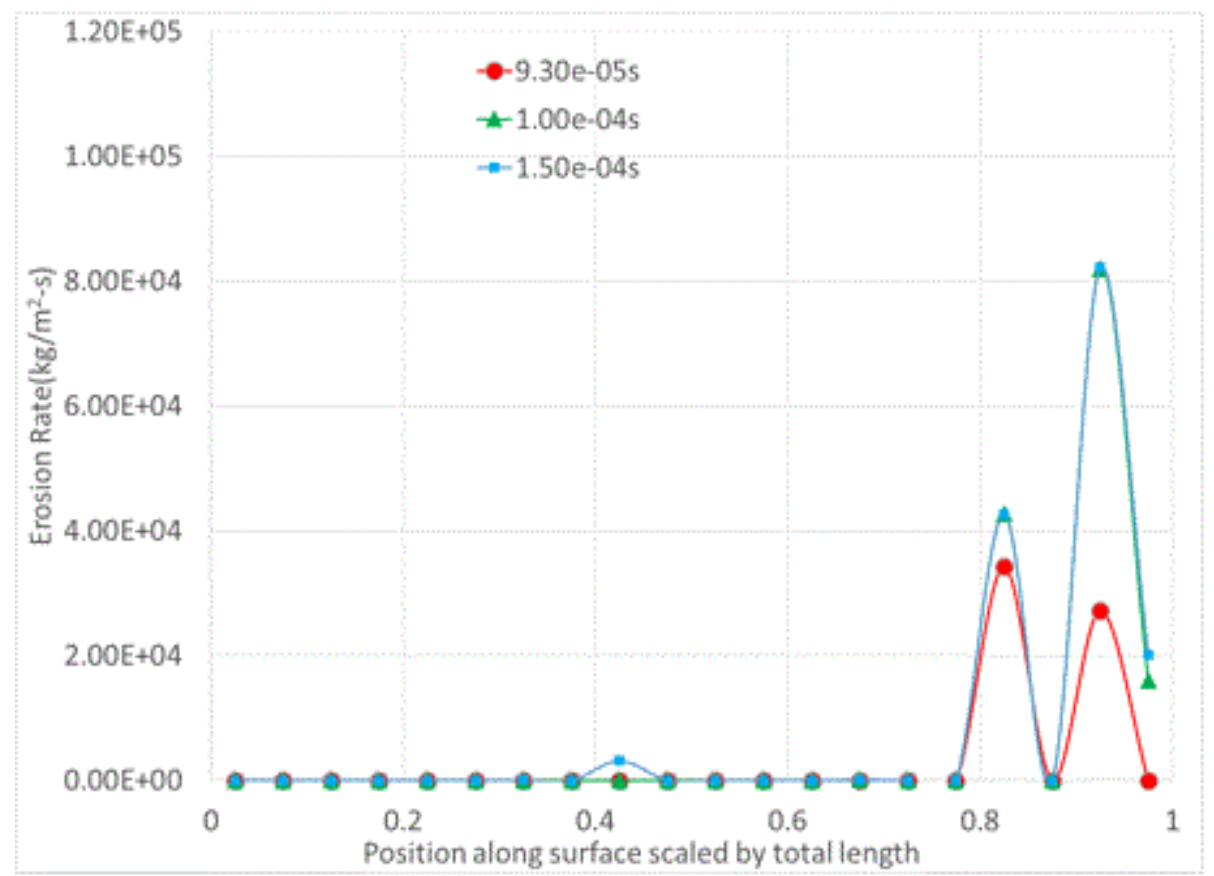

c)surface $\mathrm{S} 10$

Figure 24. Erosion rate distributions along indicated surfaces for baffle configuration(continued) 


\section{UNCLASSIFIED}

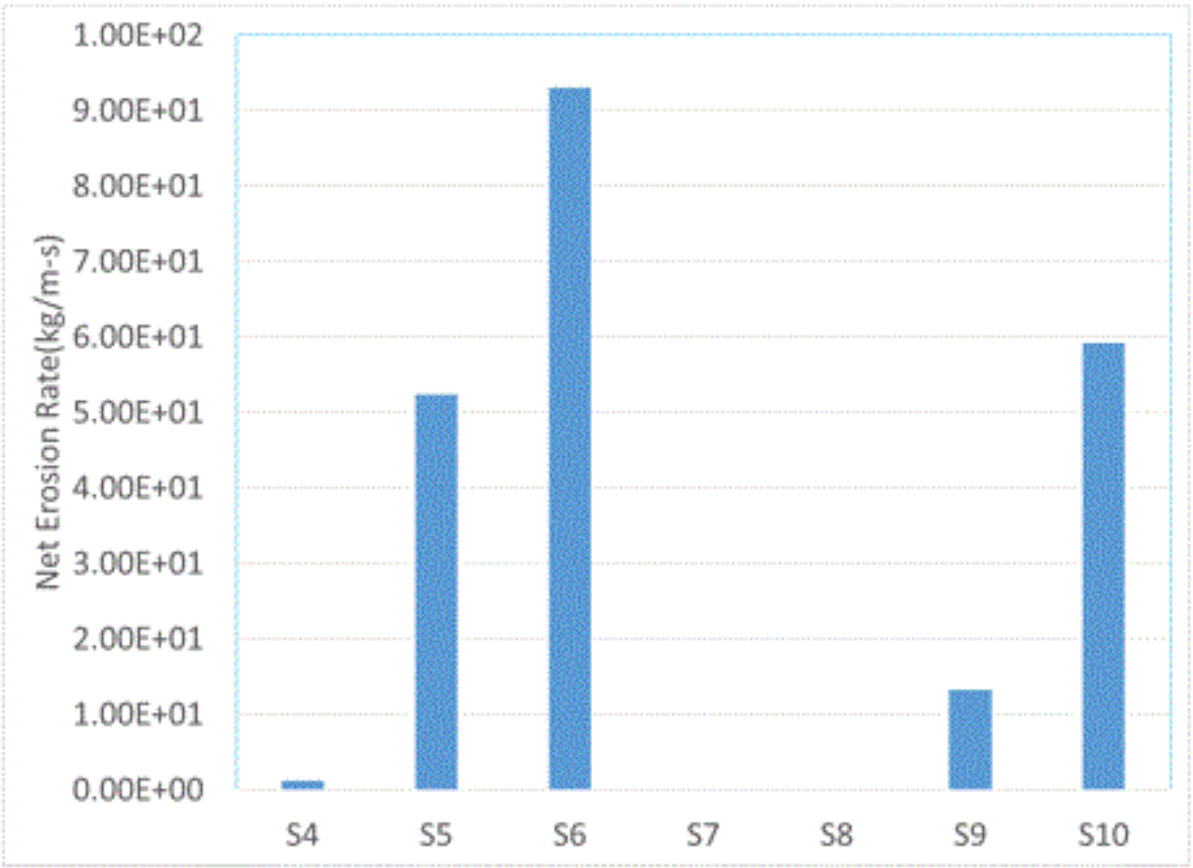

d)Net erosion rate development

Figure 24. Erosion rate distributions along indicated surfaces for baffle configuration

Table 1. Parameters for erosion rate model - 300 $\mu \mathrm{m}$ diameter sand impacting AL 6061

\begin{tabular}{ll} 
Parameter & Value \\
\hline $\mathrm{H}_{\mathrm{v}}(\mathrm{GPa})$ & 1.12 \\
$\mathrm{~F}_{\mathrm{S}}$ & 1.00 \\
$\mathrm{f}$ & 2.19 \\
$\mathrm{n}_{1}$ & 0.50 \\
$\mathrm{n}_{2}$ & 2.50 \\
$\mathrm{n}_{3}$ & 0.50 \\
$\mathrm{C}$ & $3.28 \mathrm{E}-07$
\end{tabular}




\section{UNCLASSIFIED}

\section{List of Figures}

Figure 1. Sand particles exiting a flow path and impacting fixed perpendicular Aluminum plate

Figure 2. Sand particles impacting Aluminum surfaces for three different system configurations, $272300 \mu \mathrm{m}$ diameter particles

Figure 3. Particle discretization within the computational fluid dynamics domain

Figure 4. Two colliding particles, vectors defined

Figure 5. Pressure contours demonstrating effect of damping in contact model

Figure 6. Rotation of particles as they roll along a fixed wall

Figure 7. Cohesion effects for single particle interaction with wall and wall roughness element

Figure 8. Cohesion effects for multi-particle interaction with wall with roughness elements: Pressure contour sequence with pressures in $\mathrm{Pa}$.

Figure 9. Velocity and impact angle definitions

Figure 10. Velocity and pressure contours for case of $241.01 \mathrm{~mm}$ diameter particles

Figure 11. Velocity and pressure contours for case of $680.60 \mathrm{~mm}$ diameter particles

Figure 12. Velocity and pressure contours for case of $272300 \mu \mathrm{m}$ diameter particles

Figure 13. Erosion distribution at1.0e-04 seconds for indicated particle size cases

Figure 14. Velocity and pressure contours for case of $272300 \mu \mathrm{m}$ diameter particles, initial velocity of $250 \mathrm{~m} / \mathrm{s}-\mathrm{average}$ impact velocity of $430 \mathrm{~m} / \mathrm{s}$

Figure 15. Velocity and pressure contours for case of $272300 \mu \mathrm{m} \mathrm{mm} \mathrm{diameter} \mathrm{particles,} \mathrm{initial} \mathrm{velocity} \mathrm{of} 25 \mathrm{~m} / \mathrm{s}-$ average impact velocity of $530 \mathrm{~m} / \mathrm{s}$

Figure 16. Velocity and pressure contours for case of $272300 \mu \mathrm{m}$ diameter particles, initial velocity of $10 \mathrm{~m} / \mathrm{s}-$ average impact velocity of $620 \mathrm{~m} / \mathrm{s}$

Figure 17. Erosion distribution at $9.0 \mathrm{e}-05$ seconds for indicated particle velocity cases

Figure 18. Net erosion rate as a function of the average impact velocity

Figure 19. Pressure contours $(\mathrm{Pa})$ for diverging/converging section

Figure 20. Erosion rate distributions along indicated surfaces for diverging/converging section

Figure 21. Pressure contours $(\mathrm{Pa})$ for by-pass configuration

Figure 22. Erosion rate distributions along indicated surfaces for by-pass configuration

Figure 23. Pressure contours $(\mathrm{Pa})$ for baffle configuration

Figure 24. Erosion rate distributions along indicated surfaces for baffle configuration

\section{List of Tables}

Table 1. Parameters for erosion rate model - 300 $\mu \mathrm{m}$ diameter sand impacting AL 6061 